\title{
DIRECT NUMERICAL SIMULATION OF LIQUID FLOW IN A HORIZONTAL MICROCHANNEL
}

A THESIS SUBMITTED TO

THE GRADUATE SCHOOL OF NATURAL AND APPLIED SCIENCES $\mathrm{OF}$

MIDDLE EAST TECHNICAL UNIVERSITY

BY

CENK EVREN KÜKRER

IN PARTIAL FULFILLMENT OF THE REQUIREMENTS FOR

THE DEGREE OF MASTER OF SCIENCE

IN

MECHANICAL ENGINEERING

AUGUST 2005 
Approval of the Graduate School of Natural and Applied Sciences

\section{Prof. Dr. Canan Özgen Director}

I certify that this thesis satisfies all the requirements as a thesis for the degree of Master of Science.

Prof. Dr. Kemal İder

Head of Department

This is to certify that we have read this thesis and that in our opinion it is fully adequate, in scope and quality, as a thesis for the degree of Master of Science.

Asst. Prof. Dr. İlker Tarı

Supervisor

Examining Committee Members

Asst.Prof. Dr.Derek Baker

(METU, ME)

Asst.Prof. Dr.İlker Tarı

(METU, ME)

Asst.Prof. Dr.Cüneyt Sert

(METU, ME)

Dr.Almıla Yazıcıoğlu

(METU, ME)

Dr.Ayhan Yilmazer

(Hacettepe Univ., NEE) 
I hereby declare that all information in this document has been obtained and presented in accordance with academic rules and ethical conduct. I also declare that, as required by these rules and conduct, I have fully cited and referenced all material and results that are not original to this work.

Name, Last name : Cenk Evren KÜKRER

Signature 


\title{
ABSTRACT \\ DIRECT NUMERICAL SIMULATION OF LIQUID FLOW IN A HORIZONTAL MICROCHANNEL
}

\author{
Kükrer, Cenk Evren \\ M.S., Department of Mechanical Engineering \\ Supervisor: Asst. Prof. Dr. İlker Tarı
}

August 2005, 115 Pages

Numerical simulations of liquid flow in a micro-channel between two horizontal plates are performed. The channel is infinite in streamwise and spanwise directions and its height is taken as $3.1 \times 10^{-4} \mathrm{~m}$, which falls within the dimension ranges of microchannels. The Navier-Stokes equations with the addition of Brinkman number $(\mathrm{Br})$ to the energy equation are used as the governing equations and spectral methods based approach is applied to obtain the required accuracy to handle liquid flow in the microchannel. It is known for microchannels that $\mathrm{Br}$ combines the effects of conduction and viscous dissipation in liquids and is also a way of comparing the importance of latter relative to former. The present study aims to simulate the unusual behavior of decreasing of $\mathrm{Nu}$ with increasing $\mathrm{Re}$ in the laminar regime of microchannels and to show that $\mathrm{Br}$ can be introduced to explain this unexpected behavior. Consequently, it is seen at the end of the results that secondary effect of the $\mathrm{Br}$ is observed for the singlephase convective heat transfer. Therefore, a laminar flow of a liquid in a microchannel shows different characteristics compared to a similar flow in a macrochannel. To observe the differences, three different cases are run over each of a range of Reynolds numbers: one with no axial conduction assumption that corresponds to a case similar to macrochannel flow, another case with axial conduction included in the energy equation to simulate one of the main 
differences and lastly a case with the inclusion of $\mathrm{Br}$ number in the governing equations.

A similar study is made for natural convection with the same numerical set-up for the same three cases. Formation of Rayleigh-Benard cells are observed for the critical numbers widely accepted in the literature. The results are compared with each other to see the effects of axial conduction and $\mathrm{Br}$ inclusion, in addition to Ra for natural convection.

Keywords: Microchannel flow, DNS, forced convection, horizontal channel heated from below, Brinkman number, natural convection and Rayleigh-Benard cells. 


\title{
ÖZ
}

\section{SIVI AKIŞKANLARIN DÜZLEMSEL MİKRO KANALDA DOĞRUDAN SAYISAL SIMMÜLASYONU}

\author{
Kükrer, Cenk Evren \\ Yüksek Lisans, Makine Mühendisliği Bölümü \\ Tez Yöneticisi: Ast. Prof. Dr. İlker Tarı \\ Ağustos 2005, 115 Sayfa
}

İki düzlemsel plaka arasında oluşan mikro kanallarda bulunan sıvı akışkanların sayısal simülasyonu yapılmıştır. Kanal uzunluk ve genişlik olarak sonsuz boyutta olup yüksekliği mikro kanalların boyutsal aralığına da giren $3.1 \times 10^{-4}$ metredir. Brinkman sayısının eklenmesi ile birlikte Navier-Stokes denklemleri ana denklemler olarak kullanılmış ve mikro kanalda sıvı akışını gerekli doğrulukta modelleyebilmesi için spektral metodu temel alan bir yaklaşım kullanılmıştır. Brinkman sayısının mikro kanallarda iletim ve viskozite etkilerini birleştirdiği ve aynı zamanda birbirleri arasında önemsel karşılaştırma yapmaya yaradığı bilinmektedir. Bu çalışmada, mikrokanallarda, laminar bölgede, artan Reynolds sayısı ile beklenmeyen sekilde düşen Nusselt sayısının davranışı simüle edilmeye çalışılmış ve Brinkman sayısının ortaya konulması ile açıklanabilmesi hedeflenmiştir. Bu nedene bağlı olarak tek-fazlı iletim 1sı transferi için Brinkman sayısının ikincil etkisi gözlemlenmiştir. Farklı bir deyişle, mikro kanallardaki ile makro kanallardaki laminar akış farklılık göstermektedir. Farklılıkları gözlemlemek için, belirli aralıkta olan Reynolds sayılarında üç farklı durum incelenmiştir: makro kanallardakine benzer duruma denk gelen eksensel yönde 1s1 iletimi içermeyen durum, bir diğer durum olarak eksensel iletimin enerji denklemine en önemli farklılığı simule etmesi için eklenmiş hali ve son olarak Brinkman sayısının ana denklemler içine dahil edilmesi. 
Benzer çalışma aynı sayısal düzenek kullanılarak her üç durum için doğal konveksiyon için yapılmıştır. Literatürde de kabul görmüş olan kritik değerler için Rayleigh-Benard hücre oluşumu gözlenmiştir. Sonuçlar Brinkman sayısının ve eksensel iletimin etkilerini görmek için birbirleriyle karşılaştırılmıştır.

Anahtar Kelimeler: Mikro kanallarda akış, DNS, zorlu taşınım, alttan 1sıtmalı düzlemsel kanal, Brinkman sayısı, doğal konveksiyon, Rayleigh-Benard hücresi. 
To My Parents 


\section{ACKNOWLEDGEMENTS}

I want to express my deepest gratitude to my thesis supervisor, Dr. İlker Tar1, for his guidance, inspiration, invaluable help, and advice throughout my graduate study. I thank him for the courage he gave me whenever I need the most.

I would like to thank Dr. Zafer Dursunkaya for his inspiration, time, support and for his help whenever I face an obscure problem.

I want to thank my friends; Etkin Özen, Onur Sağ, Serkan Kasapoğlu, Enver Doruk Özdemir for their support and motivation they provided me throughout my graduate study.

Finally, I want to thank my family for their continuous encouragement and their invaluable support through my hard times. They are all my reasons. 


\section{TABLE OF CONTENTS}

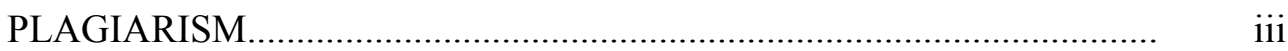

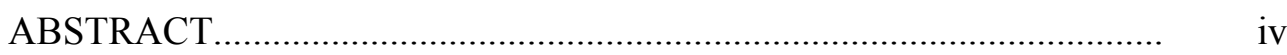

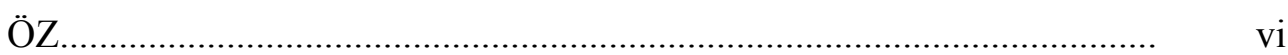

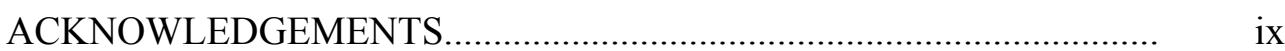

TABLE OF CONTENTS.....................................................................

LIST OF TABLES......................................................................... xii

LIST OF FIGURES....................................................................... xiii

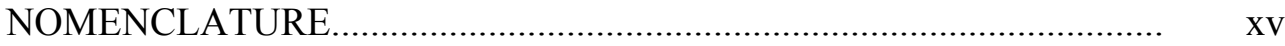

CHAPTERS

1. INTRODUCTION ..................................................................

1.1 Motivation................................................................ 2

1.2 Governing Equations...................................................... 3

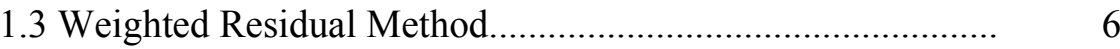

1.3.1 Spectral Methods................................................ 7

2. LITERATURE SURVEY ........................................................... 9

2.1 Forced Convection in Microchannels .............................. 9

2.1.1 Experimental and Numerical Studies................ 9

2.1.2 Brinkman Number and Axial Conduction in literature...................................................... 26

2.2 Natural Convection in Microchannels................................. 27

2.2.1 Rayleigh-Benard Cells.................................... 28

3. SPECTRAL METHOD SOLUTION OF N-S.............................. 29

3.1 Geometry, Domain and Assumptions ..................... 29

3.2 Formulation of N-S......................................... $\quad 30$

3.3 Solution of N-S Using Spectral Methods.......................... 32

3.3.1 Reformulation of the Problem............................. 32

3.3.2 Series Expansion................................................. 38

3.3.3 Temporal Discretization.................................... $\quad 39$ 
3.3.4 Spatial Discretization............................................ 40

3.4 Spectral Integration of N-S................................................ 43

3.5 Computational Procedure......................................................... 49

4. RESULTS AND DISCUSSION FOR FORCED CONVECTION

IN MICROCHANNELS................................................................

4.1 Fully Developed Velocity Distribution................................... 55

4.2 Heat Transfer Analysis......................................................... 55

4.2.1 Test Cases for Numerical Experimentation.......... $\quad 57$

4.2.2 Numerical Solution............................................... 59

4.3 Numerical Verifications........................................................... 59

4.3.1 2D vs. 3D ...................................................... 59

4.3.2 Grid Independency............................................... 61

4.4. Results and Discussion............................................................ 64

4.4.1 Comparison of the Results with Literature........... $\quad 73$

5. NATURAL CONVECTION IN MICROCHANNELS................... 89

5.1 Heat Transfer Analysis............................................................ 91

5.1.1 Numerical Formulation.......................................... 91

5.2 Numerical Verifications........................................................ 93

5.2.1 Grid Independence................................................. 93

5.2.2 Diffusion Time Scale Determination..................... 94

5.3 Results and Discussion for 2D.............................................. 94

5.3.1 Effect of Br for Natural Convection...................... 94

5.3.2 Effect of Ra on Natural Convection in

Microchannels...................................... 96

5.4 Results and Discussion for 3D.............................. 99

5.4.1 Effect of $\mathrm{Br}, \mathrm{Ra}$, and Axial Conduction for

Natural Convection....................................................... 99

6. CONCLUSION ................................................................ 105

REFERENCES......................................................................... 110 


\section{LIST OF TABLES}

Table 4.1 Selected cases for the simulation runs........................................ 59

Table 4.2 Numerical experimentation cases with corresponding nondimensional numbers........................................................... 75 


\section{LIST OF FIGURES}

Figure 1.1 The Geometry of the Problem..................................................... 4

Figure 4.1 Fully Developed Laminar Velocity Profile Inside a Microchannel Between Parallel Plates....................................... $\quad 55$

Figure $4.2 \quad \mathrm{Re}=80$ dimensionless velocities (3D)...................................... 60

Figure 4.3 $\mathrm{Re}=80$ dimensionless velocities (2D).................................... 61

Figure 4.4 $\mathrm{Re}=300$, dimensionless velocities for $64 \times 32$ mesh................... 62

Figure 4.5 $\mathrm{Re}=300$, dimensionless velocities for 128x64 mesh.................. 63

Figure 4.6 $\mathrm{Re}=300$, dimensionless velocities for 256x128 mesh............... 64

Figure $4.7 \quad \mathrm{Re}=300$ streamline contours at $\mathrm{t}^{*}=200$ with $128 \times 64$ resolution.. 65

Figure $4.8 R e=300$ temperature contours at $t^{*}=200$ with $128 \times 64$

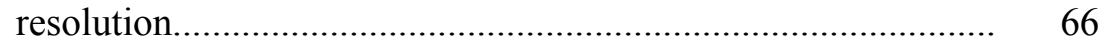

Figure 4.9 Comparison of $\mathrm{Re}=80$ no-axial conduction and $\mathrm{Br}$ (streamline) results at $t^{*}=200$ with $256 \times 128$ resolution............................... 67

Figure 4.10 Comparison of $\mathrm{Re}=80$ no-axial conduction and $\mathrm{Br}$ temperature contours at $\mathrm{t}^{*}=200$ with $256 \times 128$ resolution.............................. 68

Figure 4.11 Time change of $u$ and $v$ velocities at the middle of the channel for $\mathrm{Re}=80$ no-axial conduction case.

Figure 4.12 For $\mathrm{Re}=80$ no-axial conduction case, streamline contours at $\mathrm{t}^{*}=100,120$.

Figure 4.13 For $\mathrm{Re}=80$ no-axial conduction case, streamline contours at $\mathrm{t}^{*}=160,300$.

Figure 4.14 For $\mathrm{Re}=80$ no-axial conduction case, temperature contours at $\mathrm{t}^{*}=100,120$.

Figure 4.15 For $\mathrm{Re}=80$ no-axial conduction case, temperature contours at $\mathrm{t}^{*}=160$ and 300 ...

Figure 4.16 Plot of $\mathrm{Nu} /\left(\mathrm{Re}^{0.62} \operatorname{Pr}^{1 / 3}\right)$ vs. $\mathrm{Br}$.............................................. 76

Figure 4.17 Plot of $N u /\left(\operatorname{Re}^{0.62} \operatorname{Pr}^{1 / 3}\right)$ vs. Br, Tso and Mahulikar [10]........... 77

Figure 4.18 4.18 Plot of $\mathrm{Nu} /\left(\operatorname{Pr}^{1 / 3}\right)$ vs. Re................................................ 78

Figure 4.19 Plot of $\mathrm{Nu} /\left(\mathrm{Pr}^{1 / 3}\right)$ vs. Re, Tso and Mahulikar [10] ................... 79

Figure 4.20 Plot of $\mathrm{Nu}$ vs. Re.................................................................... 80

Figure 4.21 Plot of $\mathrm{Nu}$ vs. Re, Tso and Mahulikar [10] ............................... 81 


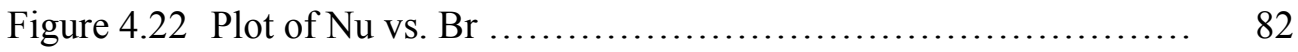

Figure 4.23 Plot of $\mathrm{Nu}$ vs. Br, Tso and Mahulikar [10].............................. 83

Figure 4.24 Plot of $\mathrm{Nu}$ vs. $\mathrm{Br}$ on a linear scale............................................ 84

Figure 4.25 Plot of $\mathrm{Nu}$ vs. Br on a linear scale, Tso and Mahulikar [10]..... 85

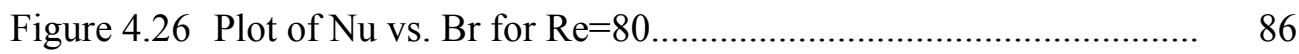

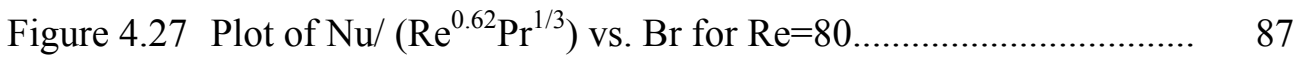

Figure 5.1 Streamline contours for $32 \times 64$ mesh and $64 \times 128$ mesh........ 93

Figure 5.2 Time change of velocity at node [2] [0] [1] for $10^{-3}$ and $10^{-4} \ldots 94$

Figure 5.3 Streamline contours for axial conduction included, $\mathrm{Br}$ and Noaxial conduction included case........................... 95

Figure 5.4 Temperature contours for axial cond. included and $\mathrm{Br}$ cases.... 96

Figure 5.5 Streamline contours for $\mathrm{Ra}=25.219$ and $\mathrm{Ra}=2000 \ldots \ldots \ldots \ldots . \quad 97$

Figure 5.6 Schematic diagram of convection cells a) Two dimensional rolls b) Hexagonal $l$ - and $g$-cells....................... 98

Figure 5.7 3D Streamline contours for axial conduction included case.... 99

Figure 5.8 3D Streamline contours for Br included in energy eqn. case... 100

Figure 5.9 3D Streamline contours for no-axial conduction case.......... 101

Figure 5.10 Dimensionless temperature sliced at $y=-0.25$ for axial conduction included case................................. 102

Figure 5.11 Dimensionless temperature sliced at $y=-1$ for no-axial conduction included case................................ 103

Figure 5.12 Dimensionless temperature sliced at $\mathrm{y}=-0.25$ for no-axial conduction included case 


\section{NOMENCLATURE}

\begin{tabular}{|c|c|}
\hline$a_{j}$ & coefficient in Eqn. 3.113 \\
\hline$b_{j}$ & coefficient in Eqn. 3.123 \\
\hline $\mathrm{Br}$ & Brinkman number, $\mu u_{m}^{2} / k \Delta T$ \\
\hline$c_{p}$ & constant pressure specific heat, $\mathrm{J} / \mathrm{kgK}$ \\
\hline$c_{j}, d_{j}$ & coefficients in Eqn. 3.123 \\
\hline$g$ & gravitational acceleration, $\mathrm{m} / \mathrm{s}^{2}$ \\
\hline G & vorticity in vertical direction \\
\hline$h$ & convective heat transfer coefficient, $\mathrm{W} / \mathrm{m}^{2} \mathrm{~K}$ \\
\hline$k$ & thermal conductivity, $\mathrm{W} / \mathrm{mK}$ \\
\hline$K n$ & Knudsen number, $\lambda / \mathrm{L}$ \\
\hline$L$ & channel height \\
\hline$L$ & length scale \\
\hline$N_{x}, N_{y}, N_{z}$ & number of intervals in spatial discretization \\
\hline $\mathrm{Nu}$ & Nusselt number, $h L / k$ \\
\hline$p$ & pressure, $\mathrm{kPa}$ \\
\hline $\operatorname{Pr}$ & Prandtl number, $v / \alpha$ \\
\hline$R a$ & Rayleigh number, $\left(\bar{L}^{3} \beta g \Delta T\right) / \alpha v$ \\
\hline$R e$ & Reynolds number, $L U / v$ \\
\hline$t$ & time \\
\hline$T$ & fluid temperature, $\mathrm{K}$ \\
\hline
\end{tabular}




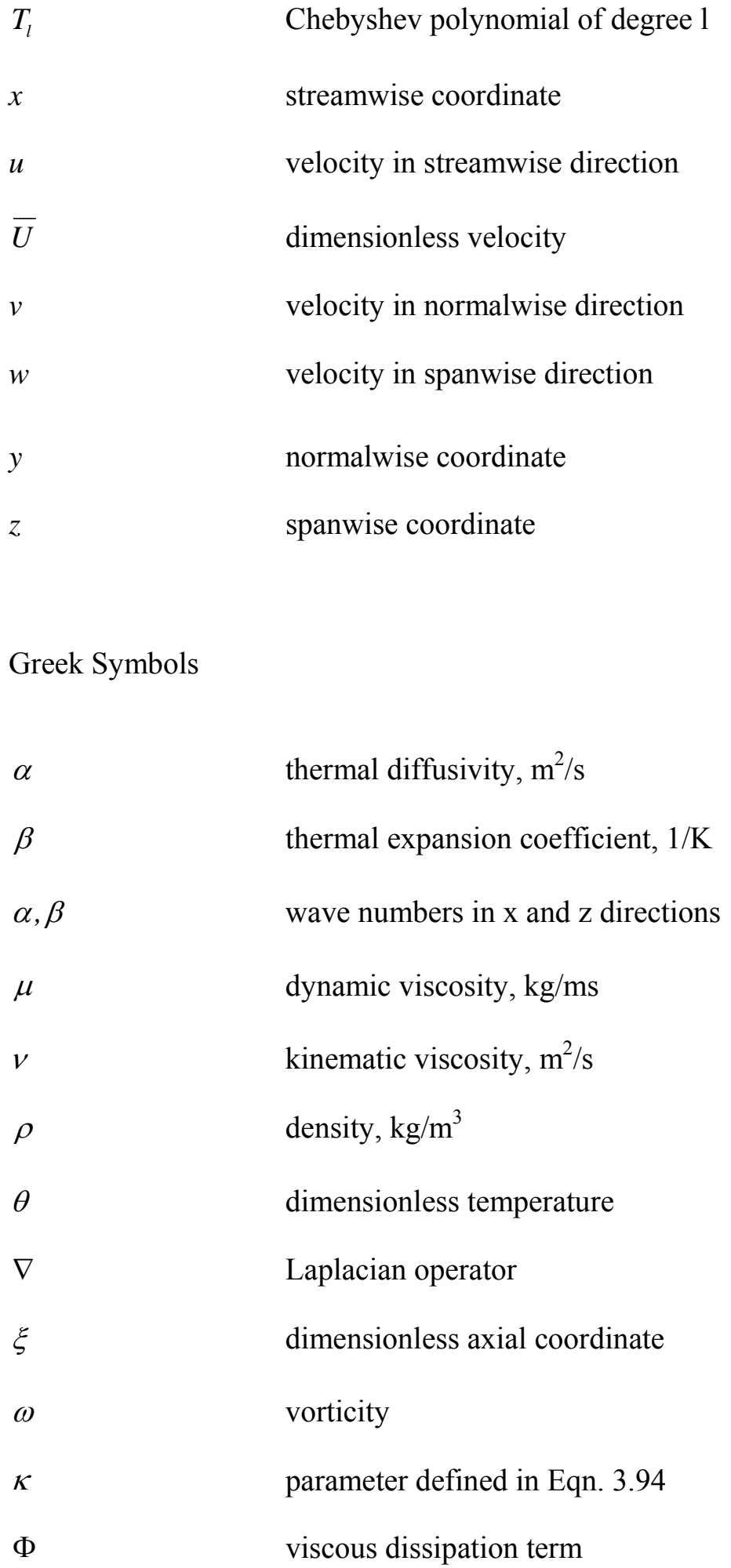




$\begin{array}{ll}\text { Subscripts } & \\ i, j, k & \text { number of collocation points } \\ i, j, k & \mathrm{x}, \mathrm{y} \text { and } \mathrm{z} \text { location } \\ m & \text { mean values } \\ s & \text { fluid properties at the surface } \\ w & \text { wall values } \\ x & \text { local values } \\ b & \text { bulk } \\ f & \text { fluid }\end{array}$

Superscript

*

dimensionless values
Abbreviations
CFD
computational fluid dynamics
DNS
direct numerical simulation
FEM
finite element method
$\mathrm{N}-\mathrm{S}$
Navier-Stokes
WRM
weighted residual method
FFT
fast fourier transform 


\title{
CHAPTER 1
}

\section{INTRODUCTION}

\begin{abstract}
Among the novel methods for thermal management of high heat fluxes, microchannels are the most effective when there is less space available for heat removal. Therefore, microchannels and microchannel heat exchangers are expected to become an integrated part of some Microelectromechanical systems (MEMS) as well as miniaturized mechanical systems. Owing to this trend, there is a newly generated interest in Computational Fluid Dynamics (CFD) simulations of microflows. As in macrochannel simulations, usually heat transfer accompanies the flow. In microchannel convective heat transfer, mainly due to small domain and low velocities, there seems to be significant differences with the macrochannel case.
\end{abstract}

Microstructure technology has many practical applications in many fields when compared with conventional channels; namely:

- Bioengineering,

- Bio-technology,

- Aerospace and

- Mini-heat exchangers.

This technology may provide new tools for examining physical phenomena more closely. Consequently, the thermal phenomena, often considered very difficult to investigate because of small length scales, might now be explored.

The classification of channels according to their hydraulic diameters $\left(D_{h}\right)$ can be summarized as: 


$$
\begin{array}{lll}
\text { Conventional Channels } & : & \mathrm{D}_{\mathrm{h}}>3 \mathrm{~mm} \\
\text { Minichannels } & : & 3 \mathrm{~mm}>\mathrm{D}_{\mathrm{h}}>200 \mu \mathrm{m} \\
\text { Microchannels } & : & 200 \mu \mathrm{m}>\mathrm{D}_{\mathrm{h}}>10 \mu \mathrm{m} \\
\text { Transitional Channels } & : & 10 \mu \mathrm{m}>\mathrm{D}_{\mathrm{h}}>0.1 \mu \mathrm{m} \\
\text { Nanochannels } & : & 0.1 \mu \mathrm{m}>\mathrm{D}_{\mathrm{h}}
\end{array}
$$

The characteristic length for the channel used in this present work is $3.1 \times 10^{-4} \mu \mathrm{m}$. The reason for using this dimension is because this is the only experimental data we have in the literature, which has the conditions same as ours.

A large number of recent investigations study the fundamentals of the microchannel flow. The researchers compare the flow and heat transfer characteristics of microchannels to conventional channels. However, many authors observe different behavior in different experimental as well as numerical studies. Therefore, there is still a need for a consensus in the heat transfer characteristics of microflows especially for the laminar region.

Because natural convection is an unexplored topic in microchannels, there is no application of it in the literature. However, for macrochannels, natural convection systems can be found in atmospheric physics, geophysics, astrophysics, or anytime there is a stratification of fluids. Interest in Rayleigh-Rayleigh-Benard system is inspired by its applications in modeling convection in the earth's mantle, and solidification of crystals surrounded by fluid.

\subsection{Motivation}

Microfluidic systems are becoming more and more important today. There are many applications of microfluidic devices that make our lives easier and healthier. Three main examples for the applications are as follows: 
1. Lab-on-chip systems: It will replace bigger machines at hospitals; therefore affects the human living standards directly.

2. MEMS: MEMS research is wide spread, especially for applied research on fluid flow and heat transfer at microscales. Some past analytical and experimental results for the flow and heat transfer characteristics in microchannels are remarkably different from those for conventional channels, like the variation of the friction factor, the heat transfer coefficient and the early transition from laminar to turbulent flow.

3. Microchannel heat exchangers: They can be used to achieve higher heat transfer coefficients, higher thermal efficiency and a lower required fluid mass flow rate. They are widely used in condensers for automobile airconditioning and will be used in evaporators and domestic airconditioning.

Therefore, microflow is a promising subject. There is a need for numerical investigations in the literature and this is the main motivation of this study.

\subsection{Governing Equations}

Navier-Stokes (N-S) Equations are used widely in the literature as the governing equations for microchannel flows. In this study, a spectral method based approach is applied to obtain the required accuracy in the solution of N-S equations for liquid flows in microchannels. For a numerical simulation to be successful, the physics of the problem should be very well understood and most of the attention should be given to the formulation. The governing equations for fluid motion, used throughout this study, are the N-S Equations, which are accepted as the most accurate way of modeling the fluid motion under certain flow conditions for conventional channels. They can also be applied for the investigation of the flow and heat transfer in microchannels, which is, however, not the case for nanochannels. 
The computer code designed, tested and used for this work is a 3-D solver for a fluid flow problem between two parallel plates, which are modeled as $\mathrm{L}_{\mathrm{x}}$ by $\mathrm{L}_{\mathrm{z}}$ plates with periodic boundary conditions in both directions. The channel spans from $y=-1$ to $y=1$ in the normal direction and is filled with liquid. The geometry of the problem is given in Figure 1.1.

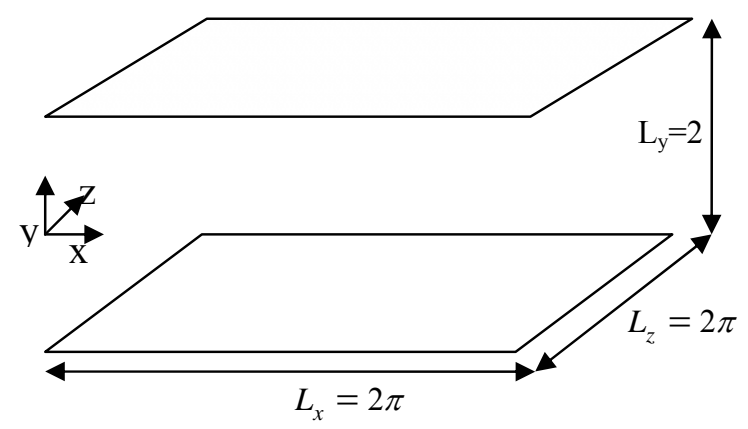

Figure 1.1. The Geometry of the Problem

The channel walls are maintained at specified temperatures. The rotational form of N-S Equations is solved using a spectral methods based approach. Three different cases are investigated:

- with axial-conduction

- without axial-conduction

- with the introduction of $\mathrm{Br}$ into energy equation are simulated.

Navier-Stokes Equations for incompressible flow without viscous dissipation: the continuity, momentum and energy equation are:

$$
\nabla \cdot U=0
$$




$$
\begin{gathered}
\frac{\partial U}{\partial t}+\omega \times U=-\nabla p+v \nabla^{2} U+\beta g T \\
\frac{\partial T}{\partial t}+U \cdot \nabla T=\alpha \nabla^{2} T
\end{gathered}
$$

With viscous dissipation energy equation becomes:

$$
\rho c \frac{\partial T}{\partial t}+\rho c(U \cdot \nabla T)=k \nabla^{2} T+\Phi
$$

where viscous dissipation term is:

$$
\Phi=2 \mu\left[\frac{1}{2}\left(\frac{\partial u}{\partial y}\right)^{2}\right]
$$

$U=(u, v, w, t)$ is the velocity field at location $X=(x, y, z), t$ is time, $p$ is the pressure, and $\alpha, v$ and $\beta$ are thermal diffusivity, kinematic viscosity, and the thermal expansion coefficient, respectively. $T$ is the fluid temperature, $g$ is the gravitational acceleration, and $\omega=\nabla \times U$ is the vorticity.

The velocity field boundary conditions are:

$$
\begin{gathered}
U(x, y, z, t)=0 \quad \text { at } \quad \mathrm{y}= \pm 1 \\
U\left(x+m L_{x}, y, z+n L_{z}, t\right)=U(x, y, z, t) \\
\frac{\partial u}{\partial x}=0 \quad \text { at } \quad \mathrm{y}= \pm 1 \\
\frac{\partial w}{\partial z}=0 \quad \text { at } \quad \mathrm{y}= \pm 1
\end{gathered}
$$

Therefore, from continuity equation: 


$$
\frac{\partial v}{\partial y}=0 \quad \text { at } \quad \mathrm{y}= \pm 1
$$

The boundary conditions for the temperature field are:

$$
\begin{gathered}
T\left(x+m L_{x}, y, z+n L_{z}, t\right)=T(x, y, z, t) \\
T(x, y, z, t)=T_{1}(x, z) \text { at } \mathrm{y}=1 \\
T(x, y, z, t)=T_{2}(x, z) \text { at } \mathrm{y}=-1
\end{gathered}
$$

The details of the solution of the governing equations are discussed in Chapter 3.

\subsection{Weighted Residual Method}

The numerical method used for the present study, a spectral method, belongs to a subgroup of weighted residual methods (WRM). WRM assumes that a solution can be approximated analytically or piecewise analytically. In general a solution to a Partial Differential Equation (PDE) can be expressed as a superposition of a set of basis functions:

$$
T(x, t)=\sum_{j=1}^{N} a_{j}(t) \phi_{j}(x)
$$

For the choice of the weight functions, $a_{j}$, three common techniques are used. Therefore, the method of calculation for $a_{j}$ determines the type of spectral method as:

- Galerkin,

- Galerkin-Tau, or

- Collocation. 
The type used in this work is the collocation method. It ensures the residual to be zero at the nodal point by using the Dirac delta functions based on a set of nodal points as the weight functions.

\subsubsection{Spectral Methods}

The numerical method used for the solution of the governing equations throughout the thesis is a spectral method. The characteristic feature of spectral methods and the intention for using them as the numerical method for this study could be stated as follows:

- It is a global approximation approach.

- The main point of using spectral methods is to try to find a solution to a differential equation by the help of a series of known, smooth functions, which covers the whole domain.

- The key point for the spectral methods is the trial functions, which are used in the truncated series expansion. They are generally selected as infinitely differentiable global functions. Trigonometric polynomials, Chebyshev polynomials and Legendre polynomials are commonly used as the trial functions.

- The "Fourier Spectral Method" is the name coming from the usage of trigonometric polynomials as the trial function for the solution of the problem.

Chebyshev polynomials used in this work for the solutions of one of the directions are also interpreted as a Fourier cosine expansion with a modification.

Spectral methods have many advantages compared to other WRMs such as:

- Spectral accuracy: The use of spectral methods is advantageous because of the fact that the addition of a few terms to sufficient number of 
coefficients of the expansion improves the accuracy and also the error decreases exponentially. An example may be the comparison between Finite Element Method (FEM) and spectral method. FEM is a low-order approximation method. It generally uses first or second order polynomial approximations. Fluid flow problems, like the one in this work, require high resolution and accuracy. This is where FEM loses its attractiveness.

- Pseudospectral methods employ spectral space only to determine spatial derivatives (much more efficient),

Some disadvantages of Spectral Methods are as follows:

- They are for global approximations and hence one has problems when dealing with geometries that have irregular domains. Problems with complex domains can be solved with the Finite Element Method, which has local trial functions. Since the problem domain can be divided into triangles or quadrilaterals, the complex domain geometry is not a problem anymore, when one can combine these shapes to form any domain.

- It does not have any variable grid resolution, which other local approximation methods (finite difference, finite volume and finite element) have.

The present study is intended to work on convective heat transfer in microchannels by direct numerical simulation. The thesis begins with a broad literature review of microchannels, Chapter 2, and continues with Chapter 3 by explaining a pseudospectral method in detail as the solution technique, spectral methods. Chapters 4 and 5 show the results for forced and natural convection respectively, for the liquid flow through a microchannel. The last chapter gives the conclusions drawn from the study. 


\section{CHAPTER 2}

\section{LITERATURE SURVEY}

The interest in flow and heat transfer in microchannels has increased rapidly during the last decade. In general, there also seems to be a shift in the focus of published articles, from descriptions of the manufacturing technology to discussions of the physical mechanisms of flow and heat transfer.

The previous studies on fluid flow in microchannels are summarized under two headings: forced convection in microchannels and natural convection in microchannels.

\subsection{Forced Convection in Microchannels}

\subsubsection{Experimental and Numerical Studies}

Due to the growing interest in micron size devices and systems, there has been a great amount of fluid flow and heat transfer studies to understand the governing principles and the physics of the flow in microchannels.

Peng and Peterson published several papers about single-phase flow in micro channels. In one of their articles [1], they investigate convective heat transfer and flow friction for water flow in microchannel structures. Single-phase forced convection heat transfer of water through small rectangular channels with the hydraulic diameter of 0.133-0.367 $\mathrm{mm}$ and different geometric configurations are investigated by experiments and their Re ranges over 50-4000. Experiments are done using water as the working fluid with an inlet temperatures between 20$45^{\circ} \mathrm{C}$ and velocities between $0.2-12 \mathrm{~m} / \mathrm{s}$. Boundary conditions are simulated as three surfaces with uniform heat flux except the top surface which is insulated. 
The authors reach the conclusion that because of the liquid temperature variations, $f$ and Re varied along the length of the plate and the transition Re number decreases as the hydraulic diameter $\left(D_{h}\right)$ decreased. Moreover, geometrical parameters like $\mathrm{D}_{\mathrm{h}}$, height to width ratio of the microchannel, the ratio of the hydraulic diameter to microchannel center to center distance, $\mathrm{D}_{\mathrm{h}} / \mathrm{W}_{\mathrm{c}}$, have important effects on heat transfer and flow characteristic. For laminar flow of water in twelve different microchannels, a correlation is found. They demonstrate that the laminar heat transfer can be improved by increasing the $D_{h}$ or decreasing $\mathrm{W}_{\mathrm{c}}$. In another paper by the same authors [2], they indicate a decrease in $\mathrm{Nu}$ with increasing $\mathrm{Re}$, where the flowing fluid used in the experiments are water and methanol which have velocities and temperatures ranging between $0.2-2.1 \mathrm{~m} / \mathrm{s}, 11-28{ }^{\circ} \mathrm{C}$ and $0.2-1.5 \mathrm{~m} / \mathrm{s}, 12-20{ }^{\circ} \mathrm{C}$ respectively. Their conclusion is for $\operatorname{Re}<400, \mathrm{Nu}$ depends on important parameters other than Re. The authors observe that the heat transfer coefficient becomes larger as the liquid temperature decreases or velocity of flow increases and microchannel size has an important role in determination of the flow mode and heat transfer dynamics. Their conclusion is that $h$ varies with $T_{w}$ because of the small size of the channel and for similar flow rates and velocities heat transfer regimes may be different for different microchannels with different sizes. Moreover they proposed that "The range of transition zone, and heat transfer characteristics of both the transition and laminar flow regimes are strongly affected by the liquid temperature, liquid velocity, microchannels size, and, hence are not only determined by Re."

Wang and Peng [3] experimentally investigate the liquid forced-convection heat transfer through microchannels. Experiments are done to investigate the singlephase forced convection of both deionized water and methanol flowing through rectangular microchannels. Free convection effects are neglected and only forced convection applies with a Re range of 70-4000 and 200-3000 for water and methanol, respectively. Their conclusion about transition and laminar regime is that the heat transfer characteristics in microchannels are quite obscure and highly complicated when compared to macrochannel cases. Liquid temperature, 
velocity and size of the microchannel affect directly the heat transfer characteristics in the laminar regime.

Tuckermann and Pease $[4,5]$ demonstrate that the heat transfer coefficient of laminar flow through microchannels may even be greater than that of turbulent flow of conventional size channels. Moreover, $\mathrm{Wu}$ and Little [6], Pfahler et al. [7] and Choi et al.[8] demonstrate that the heat transfer and flow characteristics in microchannels and microtubes differ from the experimental results of conventionally sized channels. Generally, heat transfer is enhanced as liquid temperature decreases and velocity increases and rise of heat transfer coefficient with surface temperature is bounded by the microchannel size reduction. For an extremely small channel, the heating creates a large change in liquid temperature. It is seen in the experiments that for water flow the Reynolds number is doubled with respect to inlet at the exit. Variation in heating rate or wall temperature causes Reynolds number deviations. $\mathrm{Wu}$ and Little [6] find that transition to turbulence is at lower Reynolds numbers in microchannels than conventionally sized channels. Results of their work indicate that while $\operatorname{Re}>1500$ and $700<\operatorname{Re}<1500$, data generally falls close to a straight line, for $\operatorname{Re}<700$ the relationship is quite obscure between $\mathrm{Nu}$ and $\mathrm{Re}$. They find a correlation with an empirical constant different than conventional case (Dittus-Boelter equation) by modifying the known equation $N u=0.023 \operatorname{Re}^{4 / 5} \operatorname{Pr}^{1 / 3}$ by changing the constant value $(0.023$ to 0.00805$)$. With the help of the experimental data they point out that fully developed turbulent flow is induced about $1000<\mathrm{Re}<1500$ for liquid flow in microchannels.

Peng and Wang [9] also experimentally investigate the heat transfer in flat plates with rectangular microchannels. In these experiments, methanol was used as a working fluid in rectangular shaped parallel microgrooves. Average liquid temperature and velocity varied between $14-19{ }^{\circ} \mathrm{C}$ and $0.2-1.5 \mathrm{~m} / \mathrm{s}$ respectively. They conclude that how liquid temperature or subcooling affects the occurred transition is not clear. In addition to this, for both large-scale and microchannel structures, the flow velocity, temperature and degree of subcooling are the most 
crucial parameters for heat transfer. The increase in flow velocity enhances the heat transfer. Also the inlet velocity alters the wall heat flux. The authors conclude that liquid velocity, liquid subcooling, liquid properties and geometry of microchannels have important influence on the heat transfer performance, cooling characteristics and liquid flow. Microchannel dimensions with the heat addition process causes a large liquid temperature change and the result of a large liquid temperature change is a significant alteration in thermophysical properties of the liquid. Moreover, variations of heating rate or wall temperature cause $\operatorname{Re}$ to change from $700-1000$ at the inlet to $1800-2000$ at the outlet.

Tso and Mahulikar [10] show the importance of the inclusion of Brinkman number in the heat transfer correlation for laminar flow in microchannels.

$$
B r=\frac{\mu u_{m}^{2}}{k \Delta T}
$$

The authors conclude that the relation between $\mathrm{Nu}$ and $\mathrm{Re}$ is very complicated in laminar and transition regimes and $\mathrm{Nu}$ may be function of other variables besides Re. They propose that $\mathrm{Br}$ can be a solution for the unexpected decrease of $\mathrm{Nu}$ with increase of Re in the laminar regime and no change in the transition regime. They think that viscous dissipation decides the fundamental limit to the reduction of the microchannel dimension and therefore $\mathrm{Br}$ is the measure of that limit. They indicate an important conclusion that $\mathrm{Br}$ is very important in the laminar regime because of the fact that the steep velocity and temperature gradients are maintained in microchannels, and the relative effect of viscous dissipation should be important. When comparing this concept with macrochannels, it is obvious that $\mathrm{Br}$ has no relevance in conventionally sized channels because for obtaining the same gradients large velocities are required which leads to turbulent flow, hence flattened gradients. One of the most important conclusions of their work is that when $\mathrm{Br}$ has values close to 0.25 , the effect of cooling the fluid is offset by viscous dissipation and wall heat transfer has no effect after this specific point. 
Finally, they propose that the significance of $\mathrm{Br}$ is expected to diminish for low values of $\mathrm{Br}$.

The same authors also demonstrate experimental verification of the role of Brinkman number in microchannels by using the local parameters in another paper [11]. There are two test specimens and heat flux and mass flow rate are the parameters varied. Constant wall heat flux condition exists during each performed experiment. Attention is paid to the distinction between conventionally sized channels and microchannels due to the fact that $\mathrm{Nu}$ should increase as $\mathrm{Re}$ increases in large channels but not in microchannels. In the laminar regime unusual behavior can be explained by correlating with Brinkman number in addition to $\operatorname{Pr}, \operatorname{Re}$ and dimensionless geometrical parameters. For this unusual behavior, the reported correlations do not fit with the experimental data. During former experiments in the literature, $\mathrm{T}_{\mathrm{w}}$ is not measured axially with the flow to calculate the local $\mathrm{Nu}$ variation for correlating it with local $\mathrm{Re}$, which changes with local $\mathrm{T}_{\mathrm{f}}$. Therefore the motivation is to correlate in laminar flow regime with $\mathrm{Br}$ for parameters taken locally and along the flow. The authors propose that a universal correlation with $\mathrm{Br}$ can be made for different velocities, if the data from the experiments are taken locally along the flow with constant heat flux boundary condition. Low values of $\mathrm{Br}$ are insufficient to affect fluid temperature directly. $\mathrm{Br}$ is changed along the flow. The cause for this is mainly the reduction in viscosity, and the increase in fluid temperature. So the result is drawn that the change in $\mathrm{Br}$ along the flow affects the temperature gradient along the flow. However, the variation of $\mathrm{Br}$ does not affect the flow due to changes in viscosity in conventionally sized channel because the gradients are not steep. The ratio of the fluid temperature gradient at the wall and wall-fluid temperature difference determines heat transfer coefficient. The authors claim that the temperature profile changes for a fully-developed flow and these variations in the temperature profile along the flow are captured by the variations in $\mathrm{Br}$. In contrast to conventionally sized channels, the velocity profile is changing in microchannels along the steady, incompressible flow even though the flow is fully-developed, though the mean velocity is constant still. Another important conclusion drawn 
by the author is that the temperature and velocity gradients in microchannels are steep in the laminar regime and this steep temperature gradient over the crosssection causes the viscosity to vary substantially.

Peng and co-workers $[1,2,3,9,12,13]$ obtain heat transfer coefficients increasing with Reynolds number for a laminar single-phase flow with water in microchannels, while a constant heat transfer coefficient is expected for macrochannels. Nevertheless, the heat transfer coefficient in the turbulent flow region is found to be predicted by a modified Dittus-Boelter equation. Moreover, their work demonstrates that the transition Reynolds number ranged from 200 to 700.

Yang and Webb [14] show that with a turbulent horizontal liquid flow of R-12 in rectangular mini-channels the Petukhov correlation given by Incropera and DeWitt [15] is in good agreement with their heat transfer results.

Garimella et al. [16] study developing flows in mini-channels and found a transition Reynolds number between 800 and 2000 for the heat transfer coefficient, which is relatively high when compared to microchannel work in the literature. Transition to turbulence is visible at $\mathrm{Re}=2000$ approximately. According to the authors the results are in good agreement with the conventional tubes both in laminar and turbulent region. A local, global and an averaged heat transfer coefficients are calculated. In the turbulent region global $\mathrm{Nu}$ is in agreement with Gnielinski [17] correlation, which is modified for thermal entry length effect. For $500<\operatorname{Re}<2000$, Shah and London [18] correlation for thermally developing flow is in agreement with the results. Nu slightly increases with Re. It is investigated and explained as the flow possibly does not reach a steady state profile. For $\operatorname{Re}<500$ the results are smaller than the Shah and London correlation and the interpretation is that longitudinal conduction happens with heat flux constant heating. 
Tunç and Bayazıtoğlu [19] solve the transient heat convection problem in their study. In a circular microchannel for hydrodynamically fully developed and thermally developing flow conditions with constant wall temperature by the integral transform and Laplace transform techniques, they solve the problem analytically. The authors investigate the effects of slip velocity, temperature jump and viscous heating. For their specified conditions, the results show that the viscous heating effects increase the Nusselt number. They reach the conclusion that at early stages the viscous heating is not significant because the temperature difference between the fluid and the wall at the beginning is the main driving force for heat transfer. At later stages of the flow the authors state that the values become closer and viscous dissipation becomes significant.

In another paper of Tunç and Bayazıtoğlu [20], the authors study the convective heat transfer for gaseous flow for steady state, hydrodynamically developed laminar flow in microtubes with uniform temperature and uniform heat flux boundary conditions by integral transform technique. Two important conditions are included in their study, which are namely, temperature jump at the wall and viscous heating within the medium. With a fixed $\mathrm{Br}$ number and for uniform temperature case, at specified axial lengths the effect of viscous heating is studied for developing region, reaching the fully developed Nusselt number. For both of the cases with fluid being heated or cooled, the effect of viscous heating is investigated. They show the combined effects of Brinkman number, Knudsen number, which is one of the most important parameters for gaseous flow and Prandtl number on the heat transfer. For the effect of temperature jump on the Nusselt number, the authors reach the conclusion that when only the velocity slip condition is included and temperature jump condition on the wall are not considered, the Nusselt number increases with increasing Knudsen number, which states that the velocity slip has a positive effect on the Nusselt number.

Xu et al [21] state that the mechanisms of flow and heat transfer in microchannels are still not understood clearly and there is little experimental data and theoretical analysis available in the literature to fully understand the mechanisms. They 
assume that, as the dimensions of flow channels approach the micro-level, viscous dissipation could be too significant to be neglected due to a high velocity gradient in the channel. However, the authors admit that no evidence and analysis is presented to verify such an explanation. After analyzing the effects of the viscous dissipation on the characteristics of the liquid flow in microchannels, the authors conclude that the effects become significant and influence the temperature, pressure and velocity distributions in the flow. It is strongly pointed out that the relationships between the average friction factor and the Reynolds number change when the hydraulic diameter of the microchannel is very small. The viscous dissipation effects are brought about by rises in the velocity gradient as hydraulic diameter reduces for constant Reynolds number.

For gaseous flow, Guo and Li [22] point out the larger surface to volume ratios for microchannels and microdevices. Consequently, they suggest that the factors related to surface effects have more impact to microscale flow and heat transfer. The authors conclude their work as: (1) The physical mechanisms for the size effect on the microchannel flow and heat transfer can be divided into two classifications: The gas rarefaction effect and the variations of the predominant factors influence the relative importance of various phenomena on the flow and heat transfer as the characteristic length decrease, even if the continuum assumption is still valid. (2) The size effect on the flow and heat transfer in MEMS should be largely attributed to the variation of the predominant factors in the flow and heat transfer processes. Due to a large surface to volume ratio, factors related to surface effects have more impact to the flow and heat transfer at small scales. Among these are: (a) surface friction induced flow compressibility, which makes the fluid velocity profiles; (b) surface roughness, which is likely responsible for the early transition from laminar to turbulent flow and the increased friction factor and Nusselt number; (c) the importance of viscous force in natural convection, which modifies the correlation between $\mathrm{Nu}$ and $\mathrm{Ra}$ for natural convection in a microenclosure; and (d) other effects, which include channel surface geometry, surface electrostatic charges, and axial heat conduction 
in the channel wall. All these factors may cause the flow and heat transfer behaviors in microchannels differ from that at conventional scales.

Mala and Li [23] study experimentally the water flow through microtubes with diameters ranging from 50 to $254 \mu \mathrm{m}$. For the material of the microtubes, fused silica and stainless steel are used. To analyze the flow, pressure drop and flow rates are measured. The experimental results indicate significant departure of flow characteristics from the predictions of the conventional theory for microtubes with smaller diameters. For microchannels the authors indicate that the experimental results are in rough agreement with the conventional theory and for lower Re, the required pressure drop is approximately the same as predicted by the Poiseuille flow theory. Nevertheless, as Re increases, there is a significant increase in pressure gradient compared to that predicted by the Poiseuille flow theory which means the friction factor is higher than for the conventional approach. Their results indicate material dependence of the flow behavior due to different surface roughnesses. The authors propose the roughness-viscosity model to interpret their experimental data. They define two viscosity parameters namely, dynamic and roughness viscosity and suggest that the additional momentum transfer caused by the increase due to roughness can be accounted for by introducing this roughness-viscosity parameter.

Li et al. [24] perform a number of experiments to investigate the heat transfer characteristics of water flowing through trapezoidal silicon microchannels. The channels have a hydraulic diameter ranging from 62 to $169 \mu \mathrm{m}$. The authors solve a conjugate heat transfer problem involving simultaneous determination of the temperature field in both the solid and the fluid regions. The experimental results are compared with the numerical predictions and a significant difference is seen. The comparison results indicated that the experimentally determined Nusselt number is much lower than that given by the numerical analysis. This difference may be due to the effects of surface roughness of the microchannel walls. Based on a roughness-viscosity model established in their previous work, a 
modified relation, which accounts for the roughness-viscosity effects is proposed to interpret the experimental results. By using the roughness-viscosity model, a good agreement between the experimental data and the predictions from the modified relationship is found by the authors.

Sobhan and Garimella [25] make a good review of the literature. A compilation and analysis of the results from investigations on fluid flow and heat transfer in microchannels and minichannels and microtubes in the literature is presented in their review, with experimental results and theoretical predictions. The authors demonstrate and discuss the anomalies and deviations from the behavior expected for conventional channels, both in terms of the frictional and heat transfer characteristics. Their comparative study points to differences between the flow and heat transfer in microchannels and conventional size channels. Moreover, the authors show that the information in the literature does not point to reasons for unusual trends seen in microchannel flows. They agree that there is no evidence that continuum assumptions are violated for the microchannels, most of which have hydraulic diameters of $50 \mu \mathrm{m}$ or more and analysis based on Navier-Stokes and energy equations would be expected to model the phenomena observed as long as the experimental conditions and simulations are made correctly. The authors think that the difference may be due to entrance and exit effects, differences in surface roughness in the different microchannels investigated, nonuniformity of channel dimensions, nature of the thermal and flow boundary conditions, and errors in instrumentation, measurement, and measurement locations. Given the diversity in the results in the literature, a reliable prediction of the heat transfer rates and pressure drops in microchannels is not currently possible for design applications such as microchannel heat sinks.

Palm [26] makes a broad review about heat transfer in microchannels. For singlephase flow he concludes that there seem to be no general agreement for the diameters that classical theory can be applied for determining friction factors and heat transfer coefficients in microchannels. The author points out that several authors have shown that transition from laminar to transition and turbulent flow 
starts at lower Reynolds numbers than expected for larger hydraulic diameters and that the critical Reynolds number decreases with decreasing hydraulic diameter and the reported friction factors are both above and below predictions of classical laminar theory. Moreover, it is seen that the deviations are in most cases smaller than thirty percent, although deviations of more than a hundred percent can be seen in the literature. One of the main points seen in the work is that for the heat transfer there are several reports indicating slightly higher Nusselt numbers in turbulent flow than expected from the conventional theory correlations. However, in laminar flow both higher and lower Nusselt numbers have been reported. The author suggest that there can be many reasons for these deviations: surface roughness effects, entrance effects, changing fluid properties (such as viscosity), electric double-layer effects, transport effects and slip flow. The deviations in experimental results can be caused by the difficulties in accurately determining hydraulic diameters and fluid and surface temperatures in microchannels.

For evaporation the author concludes that flow boiling is governed mainly by nucleate boiling mechanisms in the diameter range below $4 \mathrm{~mm}$. Below a specified hydraulic diameter the data of Peng and co-workers show a new phenomenon, fictitious boiling. The characteristic for this phenomenon is that no bubbles are detected, but the heat exchange and temperature differences are as in nucleate boiling.

And for condensation in microchannel flows he states that very little data are available in the literature concerning condensation inside microchannels and thought that surface tension effects should be expected to be more dominant, while the influence of gravity is expected to be smaller than for larger tubes.

Koo and Kleinstreuer [27] study the effects of viscous dissipation on the temperature field and ultimately on the friction factor using dimensional analysis and experimentally validated computer simulations. Water, methanol and isopropanol are used as the working fluids in different conduit geometries. The 
authors demonstrate that for microconduits, viscous dissipation is a strong function of the channel aspect ratio, Reynolds number, Eckert number, Prandtl number and conduit hydraulic diameter. Consequently they think ignoring viscous dissipation could affect accurate flow simulations and measurements in microconduits. The authors show that by comparing the magnitude of each term in the governing heat transfer equation, the viscous dissipation effect on the friction factor is found to increase as the system size decreases. For the viscous dissipation term to be important for water flow in a tube the hydraulic diameter should be less than $50 \mu \mathrm{m}$ and it should definitely be taken into consideration for all experimental and computational analyses. The important non-dimensional parameters for seeing the obscure characteristic of viscous dissipation are the Reynolds number and the Brinkman number (from another point of view, the Eckert number and the Prandtl number). Viscous dissipation effects may be very important for fluids with low specific heat capacities and high viscosities, even in relatively low Reynolds number flows. The authors think that because of the fluid temperature variations, the effect of viscosity change on viscous dissipation is found to be measurable for flows with a small hydraulic diameter. Moreover, especially for liquids as the fluid temperature increases the viscous dissipation effect decreases. The aspect ratio of a channel, namely height vs. width, plays an important role in viscous dissipation. The authors observe that as the aspect ratio deviates from unity, the viscous dissipation effect increases. One of the most important conclusions of the authors is that viscous dissipation increases rapidly with a decrease in channel size and hence should be considered along with imposed boundary heat sources and ignoring the viscous dissipation effect could ultimately affect friction factor measurements for flows in microchannels.

Morini et al. [28] performed a theoretical study calculating the temperature distribution in the cross-section of a rectangular duct, under the conditions of Newtonian and incompressible fluid, fully developed steady-state laminar flow. They solve the governing equations resorting to the finite Fourier transform. The temperature distributions are demonstrated and the results compared with the 
literature. The viscous dissipation is responsible for a power generation that for a particular Brinkman number allows the wall heat flux to vanish. The authors study the effects of viscous dissipation and wall heat flux. They show that as the Brinkman number increases, the bulk temperature increases, the wall temperature gradient decreases and the temperature gradient goes to zero on the duct perimeter; therefore the authors conclude that as $\mathrm{Br}_{\mathrm{w}}$ tends to infinity, the Nusselt number tends to zero. It is observed in the work that the temperature gradient at the walls decrease when the Brinkman number increase. Even though the authors thought that for a constant value of $\mathrm{Br}$, the viscous dissipation power increases as the duct thickness decreases, one can not clearly see the effect of the viscous dissipation from the results.

Larrode et al. [29] study the influence of rarefaction on heat transfer in circular tubes. They try to identify the similarities with the classical Graetz problem with a spatial rescaling factor $\rho_{s}$, which is a measure of rarefaction through its dependence on the Knudsen number. They find that heat transfer depends both on the degree of rarefaction and on the surface accommodation coefficients and the temperature jump at the wall, ignored in recent investigations, is found to be of essential importance in the heat transfer analysis. The authors work with a novel uniform asymptotic approximation to high-order eigenfunctions. The authors show that heat transfer depends on two parameters: the product $\beta_{v} K n$, which is a measure of the degree of rarefaction, and $\beta$ which is a function of the surface accommodation coefficients. Determining the effect of the temperature jump at the wall is essential for them in the heat transfer analysis. Their results agree with previous results to some extent on increased heat transfer when the temperature jump at the wall is neglected, however, with a limitation. One of the most important conclusions drawn from their work is that the heat transfer was found to increase or decrease with increasing rarefaction depending on whether $\beta<1$ or $\beta>1$, respectively and for a given $\mathrm{Kn}$ heat transfer decreases with increasing $\beta$. Their results are interpreted by noting that under slip-flow conditions gradients at the wall are smaller than in continuum flow due to the velocity slip and the 
temperature jump. In addition to that the authors develop a new uniform asymptotic approximation to the eigenfunctions of the Graetz problem, a weighted asymptotic approximation, which gives improved results. The asymptotic expressions are used to determine the heat transfer close to the entrance, a region where rarefaction effects are found to be more obscure and important.

Tselj et al. [30] work experimentally and perform a numerical analysis to evaluate heat transfer characteristics of water flowing through triangular silicon micro-channels with hydraulic diameter of $160 \mu \mathrm{m}$ in the range of Reynolds number from 3.2 to 64 . They demonstrate that the bulk water temperature does not change linearly along the channel. Both water and heated surface temperatures do not change monotonously and there are significant changes in the temperature gradient in the flow direction. The authors think that the nonmonotonous behavior of fluid and heated wall temperatures is due to high values of axial heat fluxes in the silicon wafer. Their experimental results of temperature distribution on the heated wall agree with the numerical predictions. The authors have an interesting but important proposal that the behavior of the Nusselt number along the channel has a singular point and at this specific point, the difference between the temperatures on the wall and the bulk water becomes negative and the flux changes the sign and are directed from the fluid to the wall. Consequently, the singular point shifts closer to the channel outlet with an increase of the Reynolds number. The authors use the conventional NavierStokes and energy equations as their governing equations and they thought that under conditions of their study the dissipation effects can be neglected.

Koo and Kleinstreuer [31] observe liquid microchannel flows experimentally and results of numerical experiments concerning channel entrance, wall slip, nonNewtonian fluid, surface roughness, viscous dissipation and turbulence effects on the friction factor. They classify the numerical findings in three groups. Flow instabilities, viscosity changes as the causes of deviations from the conventional 
flow theory for macrochannels and the third group devoted to studies that have no measurable differences between micro and macro fluid flow behaviors. They point out that based on numerical friction factor analyses, the entrance effect should be taken into account for any microfluidic system and it is definitely a function of channel length, aspect ratio and the Reynolds number. The authors agreed on a general fact that the wall slip effect is negligible for liquid flows in microconduits. Significant surface roughness effects are a function of the Darcy number, the Reynolds number and cross-sectional configurations. They think that channel-size effect on viscous dissipation turns out to be important for conduits with hydraulic diameter less than a hundred $\mu \mathrm{m}$. They propose that conventional theory, selectively augmented with auxiliary models, can readily predict benchmark experimental results for liquid flows in microconduits.

A modified Graetz methodology is studied by Kuznetsov et al. [32] to investigate the thermal development of forced convection in a circular duct filled by a saturated porous medium. They hold walls at constant temperature, and include the effects of longitudinal conduction and viscous dissipation. The so-called Brinkman model is employed. The analysis leads to expressions for the local Nusselt number, as a function of the dimensionless longitudinal coordinate and other parameters such as: Darcy number, Péclet number (Pe), Brinkman number. They investigate the effect of adding an axial conduction term and a viscous dissipation term to the thermal energy equation for the problem of forced convection in a duct of circular cross- section. The authors observe that in the absence of viscous dissipation, the developing Nusselt number varies little with the Darcy number but increases as Pe decreases. Even a small amount of viscous dissipation causes a substantial increase in the value of the Nusselt number for the case of constant-temperature boundary conditions. And they point out strongly that it is not so for the case of constant-flux boundary condition.

In another paper of Kuznetsov et al. [33], they work on fully developed forced convection in a parallel plate channel filled by a saturated porous medium, with boundary conditions of uniform temperature or at uniform heat flux, with the 
effects of viscous dissipation and flow work included and solving analytically. The Brinkman model is employed. The analysis is connected to expressions for the Nusselt number, as a function of the Darcy number and Brinkman number. After investigating the effect of various models for the effect of viscous dissipation the authors see that for small Darcy numbers, the difference between these models is negligible, but for larger Darcy number the difference is substantial.

An analytical study made by Morini [34] deals with a rigorous solution for the temperature field and the Nusselt numbers in the fully developed thermal region of rectangular ducts, wherein a laminar fully developed velocity profile occurs. The boundary condition of their work is a constant wall heat flux with a constant peripheral wall temperature. The author presents the results in terms of temperature profiles and Nusselt numbers, considering all the possible combinations of heated and adiabatic walls of the rectangular cross section and a comparison with the numerically evaluated Nusselt numbers found in literature is made.

Dhir et al. [35] study both single-phase forced convection and subcooled and saturated nucleate boiling experiments in small rectangular channels using FC-84 as test fluid. Their set up has a hydraulic diameter $D_{h}=0.75 \mathrm{~mm}$ and the experiments are performed with the channels oriented horizontally and uniform heat fluxes applied at the top and bottom surfaces. The parameters that are varied during the experiments included the mass flow rate, inlet liquid subcooling, and heat flux. The authors calculate the heat transfer coefficients for both singlephase forced convection and flow boiling based on the measured temperatures, pressure drops, and the overall energy balance across the test section in addition to the single- and two-phase pressure drop across the channels. They end up with correlations developed for two-phase flow pressure drop under subcooled and saturated nucleate boiling conditions, for subcooled flow boiling heat transfer and for saturated flow boiling heat transfer. 
One of the benchmark papers summarizing the previous works is the study of Ameel et al. [36]. The authors make a broad review of the past literature and summarized their work. They point out that slip flow gas data indicate for microchannel flow an approximate $60 \%$ reduction in $f$ at the same Re compared to conventional channel flows and recent available data for other types of flows are inconclusive because of the fact they appear both above and below the theoretical works. The authors agree on the fact that there is a need for additional experimental investigations over a wider Re spectrum but with much attention paid to well-characterized dimensions, surface roughness and thought that conventional theory correlations can made good estimations for microflows to some extent.

Weisber et al. [37] focus on the heat exchangers mainly used for cooling of electronic chips. They use water as their working fluid and tried to eliminate the approximations used in previous studies. The important part of their work is to solve the heat transport problem both in solid and liquid by neglecting the axial conduction in solid and the liquid. Their Reynolds number is relatively low (around 100) which falls within the limit of the laminar region that is widely accepted by many researchers of this subject. Their investigation concludes that their results are in rough agreement with experimental observations except for some special cases.

Beşkök et al. [38] investigate mixed electroosmotic pressure driven flows of Tjunction geometry as well as straight channels by comparing their numerical work with analytical results. Their work includes solving the Navier-Stokes and Poisson-Boltzman equations with the help of $h / p$ type spectral element method. The author dealt mainly with electrokinetic forces. They investigate materials with very strong and negligible electroosmotic effects in straight channels and show that $\mathrm{T}$-junction acts as an electroosmotically-actuated microvalve. 


\subsubsection{Importance of Brinkman Number and Axial Conduction in the Literature}

The importance and definitions of Brinkman number in microchannels can be found widely in the literature. In this section, the definitions and relative importance of $\mathrm{Br}$, hence viscous dissipation term, compared to other terms in Navier-Stokes equations is presented. Tso and Mahulikar have several studies concerning this crucial non-dimensional number of microflows.

Brinkman number represents the relative importance of viscous heating to fluid conduction. This dimensionless number is not widely seen in conventionally sized channels because the variation of viscosity is generally negligible as a result of smaller length-to-diameter ratios. The definition of $\Delta T$ also changes from one author to another because of the difference in formulations. The one used in our study is the difference between the lower wall and the upper wall which is arising from our formulation.

The effects of the Brinkman number can be grouped into two based on its order of magnitude. Relatively low values of $\mathrm{Br}$ (less than unity) are far from affecting fluid temperature due to viscous dissipation which is called as secondary effect of $\mathrm{Br}$ in the study of Tso and Mahulikar [11]. However, when $\mathrm{Br}$ is higher (more than unity) it affects the fluid temperature directly as a result of the higher values of the viscous dissipation term in the energy equation. This effect of the higher values of the $\mathrm{Br}$ is called primary effect of $\mathrm{Br}[11]$.

In the laminar regime $\mathrm{Br}$ is an important parameter. There exists steep velocity and temperature gradients which cause viscous dissipation term to be crucial even if it is not the case for turbulent and transition regimes.

One of the reasons of deviation of microflows from macroflows is mentioned in the literature as axial conduction. It is neglected in the energy equation of the flow through conventionally sized channels because of its low order of magnitude 
[39]. For laminar flow in microchannels the Reynolds number is kept within the range 80-300 in the present study. Therefore the flow is low Reynolds number flow which indicates that axial conduction is very crucial. As it can easily be recognized in the literature [40], as Reynolds number goes to infinity, which means high Eckert number, the importance of axial conduction becomes weaker and weaker which is generally the case in conventionally sized channels because of high transition Reynolds numbers.

\subsection{Natural Convection in Microchannels}

Unfortunately, there seems to be no literature about heat transfer with free convection in microchannels. Consequently, the present work is the very first work investigating this phenomenon and would help to understand the studies, which will be done in the future.

The only literature related to natural convection in microchannels is the work of Guo and Li [45]. The authors review the size effects on microscale single-phase fluid flow and heat transfer. Their work is actually for gaseous flow. They think that due to the larger surface to volume ratio for microchannels, factors related to surface area have more impact to the microscale flow and heat transfer. Additionally, they believe the importance of viscous force in natural convection modifies the correlation between $\mathrm{Nu}$ and $\mathrm{Ra}$ for natural convection in a microenclosure and, other effects, such as the axial heat conduction in the channel wall, the channel surface geometry, and measurement errors as well, could lead to different flow and heat transfer behaviors from that at conventional scales.

In our bottom heated channel, it is expected to observe Rayleigh-Benard cells after a certain critical Rayleigh Number $(\mathrm{Ra})$ is exceeded. 


\subsubsection{Rayleigh-Benard Cells}

For Rayleigh-Benard cells to form, one should consider a thin layer of liquid between two large parallel plates. The properties of the system are homogeneous if the system is in equilibrium, with the liquid and the two plates at the same temperature and the liquid motionless. The bottom plate is heated slowly. The heat will pass from the bottom plate to the liquid and will be transferred through the liquid to its upper layer by conduction. In thermal conduction there is no bulk motion of the liquid but rather a greater thermal motion of the molecules that causes the transfer of heat from the warmer layers to adjacent cooler layers. However, if temperature of the bottom layer is increased, a stage is reached where the liquid bouyancy overcomes its viscosity, which means the internal friction that opposes movement, and begins to undergo bulk motion. The critical non-dimensional number, $\mathrm{Ra}$, has a value of 1708 [15], which results in a transport of heat by convection currents. What is known is that the currents are not random but rather they lead to the formation of patterns. Often one first sees small convection cells (Rayleigh-Benard cells). In this example, the driving force is temperature; that is, temperature differences, or the transport of heat by a medium, can cause non-equilibrium structure formation on macroscopic scales. When viewed from above the cells form striped patterns, thus breaking the symmetry of the uniform state. As the temperature is raised, rolls can also appear in a perpendicular direction so that viewed from above one has square shaped structures.

One of the most important conclusions is that the formation of Rayleigh-Benard cells in laboratory experiments depend not just on the convection, but also mainly on surface tension. It is not just the difference in Rayleigh numbers that counts. 


\section{CHAPTER 3}

\section{SPECTRAL METHOD SOLUTION OF N-S}

As is mentioned in the previous chapters, the governing equations for the fluid motion are the Navier-Stokes equations (N-S). In this chapter, the work for formulating the problem will be shown. The numerical work presented here for the solution of Navier-Stokes equations was first formulated by Kim, Moin and Moser [41]. Breuer originated the spectral integration method for the Helmholtz system and developed the algorithm for the solution. Zhang and Tangborn [42] had developed and tested the N-S solver used in this work. And one can reach the details of the formulation of the problem by investigating the Zhang's work [42]. However, most of equations used in this work are taken from Tarı's study [43].

\subsection{Geometry, Domain and Assumptions}

The physical geometry of the problem presented in this work (see Figure 1.1) is a parallel plate channel where the fluid, which is water, fills the entire channel. The infinite walls are modeled as $\mathrm{L}_{\mathrm{x}}$ by $\mathrm{L}_{\mathrm{z}}$ plates with periodic boundary conditions at the boundaries in both streamwise $(\mathrm{x})$ and spanwise $(\mathrm{z})$ directions. The vertical distance between two parallel plates is determined as $\mathrm{L}_{\mathrm{y}}$. For the work presented, $\mathrm{L}_{\mathrm{y}}$ ranges from $\mathrm{y}=-1$ to $\mathrm{y}=1$ so that $\mathrm{L}_{\mathrm{y}}=2$. Buoyancy forces are involved for the problem at hand because of the temperature difference between the two parallel plates. The flow of the water is assumed to be incompressible and pressure gradients only exists in the x-direction. Boussinesq approximation is applied which results in ignoring the variations of all fluid properties other than the density, and the density variations only change the gravitational force. 


\subsection{Formulation of N-S}

The governing equations for the incompressible flow without viscous dissipation are given in Eqns. 1.1-1.3.

However, with viscous dissipation the energy equation becomes Eqn. 1.4 with Eqn. 1.5, which is the viscous dissipation term.

The velocity field boundary conditions are given in Eqns. 1.6a-d and 1.7a-c. The boundary conditions for the temperature field are Eqns. 1.8.

For obtaining the non-dimensional form of the Equations 1.1-3 some definitions

are made. Namely, $\bar{U}$ and $\bar{L}$ are used, respectively for the characteristic velocity and length scales of the problem at hand. We used the channel height $H$ as a length scale. The non-dimensional temperatures are obtained using $\Delta T=T_{\max }-T_{\min }$, which represents the maximum temperature difference, and $\theta=T-T_{m}$, where $T_{m}$ is the mean temperature of the lower wall. The nondimensional representation can be written as:

$$
\begin{aligned}
U^{*} & =\frac{U}{\bar{U}} \\
X^{*} & =\frac{X}{\bar{X}} \\
t^{*} & =\frac{U}{\bar{X}} t \\
\theta^{*} & =\frac{1}{\Delta T} \theta \\
p^{*} & =\frac{p}{\rho \bar{U}^{2}}
\end{aligned}
$$

Hence, the non-dimensional N-S without viscous dissipation becomes:

$$
\nabla \cdot U^{*}=0
$$




$$
\begin{gathered}
\frac{\partial U^{*}}{\partial t^{*}}+\omega^{*} \times U^{*}=-\nabla p^{*}+\frac{1}{\operatorname{Re}} \nabla^{2} U^{*}+\frac{R a}{\operatorname{Re}^{2} \operatorname{Pr}} \theta^{*} \frac{\rho}{g} \\
\frac{\partial \theta^{*}}{\partial t^{*}}+U^{*} \cdot \nabla \theta^{*}=\frac{1}{\operatorname{RePr}} \nabla^{2} \theta^{*}
\end{gathered}
$$

With the viscous dissipation term, only the energy equation changes:

$$
\frac{\partial \theta^{*}}{\partial t^{*}} \frac{\overline{\bar{X}}}{\bar{X}}+\bar{U}\left(U^{*} . \nabla \theta^{*}\right)=\alpha \nabla^{2} \theta^{*}+\frac{\mu}{\Delta T \rho c}\left(\frac{d u^{*}}{d y^{*}}\right)^{2} \frac{\bar{U}^{2}}{\bar{X}^{2}}
$$

Dividing all the terms by $\bar{U}$ we get:

$$
\frac{\partial \theta^{*}}{\partial t^{*}} \frac{1}{\bar{X}}+\bar{U}\left(U^{*} . \nabla \theta^{*}\right)=\frac{\alpha}{\bar{U}} \nabla^{2} \theta^{*}+\frac{\mu}{\Delta T \rho c \bar{U}} \frac{\bar{U}^{2}}{\bar{X}^{2}}\left(\frac{d u^{*}}{d y^{*}}\right)^{2}
$$

Inserting the non-dimensional length coming from the Laplacian operator:

$$
\frac{\partial \theta^{*}}{\partial t^{*}} \frac{1}{\bar{X}}+U^{*} \cdot \nabla \theta^{*} \frac{1}{\bar{X}}=\frac{\alpha}{\bar{U}^{2}} \nabla^{2} \theta^{*}+\frac{\mu}{\Delta T \rho c \bar{U}} \frac{\bar{U}^{2}}{\bar{X}^{2}}\left(\frac{d u^{*}}{d y^{*}}\right)^{2}
$$

which results in:

$$
\begin{gathered}
\frac{\partial \theta^{*}}{\partial t^{*}}+U^{*} . \nabla \theta^{*}=\frac{1}{\operatorname{Re} \operatorname{Pr}} \nabla^{2} \theta^{*}+\frac{\mu \bar{U}^{2}}{\Delta T k} \frac{1}{\frac{\rho c}{k} \bar{U} \bar{X}}\left(\frac{d u^{*}}{d y^{*}}\right)^{2} \\
\frac{\partial \theta^{*}}{\partial t^{*}}+U^{*} \cdot \nabla \theta^{*}=\frac{1}{\operatorname{Re} \operatorname{Pr}} \nabla^{2} \theta^{*}+\frac{B r}{\operatorname{Re} \operatorname{Pr}}\left(\frac{d u^{*}}{d y^{*}}\right)^{2}
\end{gathered}
$$

where

$$
R a=\frac{\bar{L}^{3} \beta g \Delta T}{\alpha v}
$$




$$
\begin{gathered}
\operatorname{Re}=\frac{\bar{L} \bar{U}}{v} \\
\operatorname{Pr}=\frac{v}{\alpha}
\end{gathered}
$$

And the fourth dimensionless number is the Brinkman number, which has been defined by Eqn. 2.1.

All variables after this point of the thesis will be denoted without an asterisk because everything will be non-dimensional.

\subsection{Solution of N-S by Using Spectral Methods}

\subsubsection{Reformulation of the Problem}

Writing the non-dimensional form of the $\mathrm{N}-\mathrm{S}$ in a reformulated way, we obtain:

$$
\begin{gathered}
\frac{\partial u}{\partial t}=-\frac{\partial p}{\partial x}+\mathrm{h}_{1}+\frac{1}{\operatorname{Re}} \nabla^{2} \mathrm{u} \\
\frac{\partial v}{\partial t}=-\frac{\partial p}{\partial y}+\mathrm{h}_{2}+\frac{1}{\operatorname{Re}} \nabla^{2} v+\frac{\operatorname{Ra}}{\operatorname{Re}^{2} \operatorname{Pr}} \theta \\
\frac{\partial w}{\partial t}=-\frac{\partial p}{\partial z}+\mathrm{h}_{3}+\frac{1}{\operatorname{Re}} \nabla^{2} \mathrm{w} \\
\frac{\partial u}{\partial t}+\frac{\partial v}{\partial t}+\frac{\partial w}{\partial t}=0 \\
\frac{\partial \theta}{\partial t}=-\left(u \frac{\partial \theta}{\partial x}+v \frac{\partial \theta}{\partial y}+w \frac{\partial \theta}{\partial z}\right)+\frac{1}{\operatorname{Re} \operatorname{Pr}} \nabla^{2} \theta+\frac{B r}{\operatorname{Re} \operatorname{Pr}}\left(\frac{d u}{d y}\right)^{2}
\end{gathered}
$$

where $h_{i}$ includes the convective terms, defined as 


$$
h_{i}=\varepsilon_{i j k} u_{j} w_{k}-\frac{1}{2} \frac{\partial}{\partial x_{j}}\left(u_{j} u_{j}\right)
$$

Applying the differential operator to $h_{i}$, the symmetric term will drop and we can rewrite $h_{i}$ as:

$$
\begin{gathered}
h_{1}=v \omega_{z}-\left(w \omega_{y} / 2\right) \\
h_{2}=w \omega_{x}-u \omega_{z} \\
h_{3}=u \omega_{y}-v \omega_{x}
\end{gathered}
$$

where $\omega_{x}, \omega_{y}, \omega_{z}$ are the vorticity components in $x, y$ and $z$ directions. We can define them as:

$$
\begin{aligned}
& \omega_{x}=\frac{\partial w}{\partial y}-\frac{\partial v}{\partial z} \\
& \omega_{y}=\frac{\partial u}{\partial z}-\frac{\partial w}{\partial x} \\
& \omega_{z}=\frac{\partial v}{\partial x}-\frac{\partial u}{\partial y}
\end{aligned}
$$

Since the buoyancy force acts in the y-direction and the pressure gradient only exists in the $\mathrm{x}$-direction, we can represent the non-linear terms in a different manner:

$$
\begin{gathered}
H_{1}=h_{1}-\frac{\partial p}{\partial x} \\
H_{2}=h_{2}-\frac{R a}{\operatorname{Re}^{2} \mathrm{Pr}}
\end{gathered}
$$




$$
\begin{gathered}
H_{3}=h_{3} \\
\mathrm{H}=\left(H_{1}, H_{2}, H_{3}\right)
\end{gathered}
$$

The fluid velocity governing equation can be written as:

$$
\frac{\partial \mathrm{U}}{\partial t}=\mathrm{H}+\frac{1}{\mathrm{Re}} \nabla^{2} \mathrm{U}
$$

taking the curl of the equation:

$$
\begin{gathered}
\frac{\partial}{\partial t}(\nabla \times \mathrm{U})=\nabla \times \mathrm{H}+\frac{1}{\operatorname{Re}} \nabla^{2}(\nabla \times \mathrm{U}) \\
\frac{\partial}{\partial t}\left(\omega_{x} \mathrm{i}+\omega_{y} \mathrm{j}+\omega_{z} \mathrm{k}\right)=\nabla \times \mathrm{H}+\frac{1}{\operatorname{Re}} \nabla^{2}\left(\omega_{x} \mathrm{i}+\omega_{y} \mathrm{j}+\omega_{z} \mathrm{k}\right)
\end{gathered}
$$

where

$$
\nabla \times \mathrm{H}=\left(\frac{\partial H_{3}}{\partial y}-\frac{\partial H_{2}}{\partial z}\right) \mathrm{i}+\left(\frac{\partial H_{1}}{\partial z}-\frac{\partial H_{3}}{\partial x}\right) \mathrm{j}+\left(\frac{\partial H_{2}}{\partial x}-\frac{\partial H_{1}}{\partial y}\right) \mathrm{k}
$$

We can create a second order differential equation for the normal component of the vorticity as:

$$
\frac{\partial G}{\partial t}=h_{g}+\frac{1}{\operatorname{Re}} \nabla^{2} G
$$

where

$$
\begin{gathered}
h_{g}=\frac{\partial H_{1}}{\partial z}-\frac{\partial H_{3}}{\partial x} \\
G=\omega_{y}
\end{gathered}
$$


Taking the second derivative of the normal component of velocity in the governing equation:

$$
\begin{gathered}
\frac{\partial v}{\partial t}=H_{2}+\frac{1}{\operatorname{Re}} \nabla^{2} v \\
h_{v}=\nabla^{2} H_{2}
\end{gathered}
$$

Combination of continuity and momentum equations:

$$
\frac{\partial H_{1}}{\partial x}+\frac{\partial H_{3}}{\partial z}=-\frac{\partial H_{2}}{\partial y}
$$

so $h_{v}$ can be rewritten as:

$$
h_{v}=\left(\frac{\partial^{2}}{\partial x^{2}}+\frac{\partial^{2}}{\partial z^{2}}\right) H_{2}-\frac{\partial}{\partial y}\left(\frac{\partial H_{1}}{\partial x}+\frac{\partial H_{3}}{\partial z}\right)
$$

The fourth order differential equation can be solved by dividing into two second order equations. First, a few definitions should be made:

$$
\nabla^{2} v=\Phi
$$

therefore the set of equations become:

$$
\begin{gathered}
\frac{\partial \Phi}{\partial t}=h_{v}+\frac{1}{\operatorname{Re}} \nabla^{2} \Phi \\
\nabla^{2} v=\Phi \\
v \quad=0 \text { at } \mathrm{y}= \pm 1 \\
\frac{\partial v}{\partial y}=0 \text { at } \mathrm{y}= \pm 1
\end{gathered}
$$


In this system, four boundary conditions are satisfied as follows:

$$
v=v_{p}+c_{1} v_{1}+c_{2} v_{2}
$$

where the particular solution $v_{p}$ and the two homogenous solutions $v_{1}$ and $v_{2}$ satisfy

$$
\begin{aligned}
& \frac{\partial \Phi_{p}}{\partial t}-\frac{1}{\operatorname{Re}} \nabla^{2} \Phi_{p}=h_{v} \\
& \Phi_{p}=0 \text { at } \mathrm{y}= \pm 1 \\
& \nabla^{2} v_{p}=\Phi_{p} \\
& v_{p}=0 \text { at } \mathrm{y}= \pm 1 \\
& \frac{\partial \Phi_{1}}{\partial t}-\frac{1}{\operatorname{Re}} \nabla^{2} \Phi_{1}=0 \\
& \Phi_{1}=0 \text { at } \mathrm{y}=1 \\
& \Phi_{1}=1 \text { at } \mathrm{y}=-1 \\
& \nabla^{2} v_{1}=\Phi_{1} \\
& v_{1}=0 \text { at } \mathrm{y}= \pm 1 \\
& \frac{\partial \Phi_{2}}{\partial t}-\frac{1}{\operatorname{Re}} \nabla^{2} \Phi_{2}=0 \\
& \Phi_{2}=1 \text { at } \mathrm{y}=1 \\
& \Phi_{2}=0 \text { at } \mathrm{y}=-1
\end{aligned}
$$




$$
v_{2}=0 \text { at } \mathrm{y}= \pm 1
$$

Solving the above equations, $c_{1}$ and $c_{2}$ are chosen to satisfy the above boundary conditions:

$$
\begin{gathered}
\frac{\partial v}{\partial y}=\frac{\partial v_{p}}{\partial y}+c_{1} \frac{\partial v_{1}}{\partial y}+c_{2} \frac{\partial v_{2}}{\partial y}=0 \text { at } \mathrm{y}= \pm 1 \\
\frac{\partial \Phi}{\partial t}=h_{v}+\frac{1}{\operatorname{Re}} \nabla^{2} \Phi \\
\nabla^{2} v=\Phi \\
\frac{\partial G}{\partial t}=h_{g}+\frac{1}{\operatorname{Re}} \nabla^{2} G \\
f+\frac{\partial v}{\partial y}=0 \\
\left.\frac{\partial \theta}{\partial t}=h_{T}+\frac{\partial^{2}}{\operatorname{Re} \operatorname{Pr}} \nabla^{2} \theta+\frac{\partial^{2}}{\partial x^{2}}\right) H_{2}-\frac{\partial}{\partial y}\left(\frac{\partial H_{1}}{\partial x}+\frac{\partial H_{3}}{\partial z}\right)^{2} \\
f=\frac{\partial u}{\partial x}+\frac{\partial w}{\partial z} \\
G=\frac{\partial u}{\partial z}-\frac{\partial w}{\partial x} \\
h_{g}
\end{gathered}
$$




$$
h_{T}=-\left(u \frac{\partial \theta}{\partial x}+v \frac{\partial \theta}{\partial y}+w \frac{\partial \theta}{\partial z}\right)
$$

and the boundary conditions can be written:

$$
\begin{gathered}
v \quad=0 \text { at } \mathrm{y}= \pm 1 \\
\frac{\partial v}{\partial y}=0 \text { at } \mathrm{y}= \pm 1 \\
G=0 \text { at } \mathrm{y}= \pm 1 \\
\theta=\theta_{1} \text { at } \mathrm{y}=1 \\
\theta=\theta_{2} \text { at } \mathrm{y}=-1
\end{gathered}
$$

and, lastly, the periodic boundary conditions in $x$ and $z$ directions.

\subsubsection{Series Expansion}

The readily driven equations can be solved with the help of the spectral method. The series expansion will be a Fourier series in the streamwise (x) and spanwise (z) directions, and Chebyshev polynomial expansion in the normal (y) direction. So the solution becomes:

$$
\begin{aligned}
& \mathrm{U}=\sum_{|j|<N_{x}|k|<N_{z}} \sum_{l=0}^{N_{y}} \hat{\tilde{u}}(j, k, l, t) \exp \left[2 \pi i\left(\frac{j x}{N_{x}}+\frac{k z}{N_{z}}\right)\right] T_{l}(y) \\
& \theta=\sum_{|j|<N_{x}} \sum_{|k|<N_{z}} \sum_{l=0}^{N_{y}} \hat{\tilde{\theta}}(j, k, l, t) \exp \left[2 \pi i\left(\frac{j x}{N_{x}}+\frac{k z}{N_{z}}\right)\right] T_{l}(y)
\end{aligned}
$$

where $T_{l}(y)=\cos (l \arccos y)$ is the Chebyshev polynomial of degree $l$ and $N_{x}, N_{y}$ and $N_{z}$ are the number of intervals in spatial discretization. $\sim$ and

symbols above a variable indicate that the variable is in Fourier space and Chebyshev space, respectively. 


\subsubsection{Temporal Discretization}

Crank-Nicholson and Adams-Bashforth are the methods used for temporal discretization. With discretization, the $\theta$ equation without viscous dissipation becomes:

$$
\frac{\theta^{n+1}-\theta^{n}}{\Delta t}=\frac{3}{2} h_{T}^{n}-\frac{1}{2} h_{T}^{n-1}+\frac{1}{\operatorname{Re} \operatorname{Pr}}\left(\frac{\nabla^{2} \theta^{n+1}-\nabla^{2} \theta^{n}}{2}\right)
$$

and with viscous dissipation:

$$
\frac{\theta^{n+1}-\theta^{n}}{\Delta t}=\frac{3}{2} h_{T}^{n}-\frac{1}{2} h_{T}^{n-1}+\frac{1}{\operatorname{Re} \operatorname{Pr}}\left(\frac{\nabla^{2} \theta^{n+1}-\nabla^{2} \theta^{n}}{2}\right)+\frac{B r}{\operatorname{Re} \operatorname{Pr}}\left(\frac{d u}{d y}\right)^{2}
$$

these can be written as:

$$
\begin{gathered}
\nabla^{2} \theta^{n+1}-\frac{2 \operatorname{Re} \operatorname{Pr}}{\Delta t} \theta^{n+1}=-3 \operatorname{Re} \operatorname{Pr} h_{T}^{n}+\operatorname{Re} \operatorname{Pr} h_{T}^{n-1}-\nabla^{2} \theta^{n}-\frac{2 \operatorname{Re} \operatorname{Pr}}{\Delta t} \theta^{n} \\
\nabla^{2} \theta^{n+1}-\frac{2 \operatorname{Re} \operatorname{Pr}}{\Delta t} \theta^{n+1}=-3 \operatorname{Re} \operatorname{Pr} h_{T}^{n}+\operatorname{Re} \operatorname{Pr} h_{T}^{n-1}-\nabla^{2} \theta^{n}-\frac{2 \operatorname{Re} \operatorname{Pr}}{\Delta t} \theta^{n}-2 \\
\theta=\theta_{1} \text { at } \mathrm{y}=1 \\
\theta=\theta_{2} \text { at } \mathrm{y}=-1
\end{gathered}
$$

So $\mathrm{G}$ equations become:

$$
\begin{gathered}
\frac{G^{n+1}-G^{n}}{\Delta t}=\frac{3}{2} h_{g}^{n}-\frac{1}{2} h_{g}^{n-1}+\frac{1}{\operatorname{Re}}\left(\frac{\nabla^{2} G^{n+1}-\nabla^{2} G^{n}}{2}\right) \\
\nabla^{2} G^{n+1}-\frac{2 \operatorname{Re}}{\Delta t} G^{n+1}=-3 \operatorname{Re} h_{g}^{n}+\operatorname{Re} h_{g}^{n-1}-\nabla^{2} G^{n}-\frac{2 \operatorname{Re}}{\Delta t} G^{n} \\
G=0 \text { at } \mathrm{y}= \pm 1
\end{gathered}
$$


and similarly equation for $v$ becomes:

$$
\begin{gathered}
\nabla^{2} \Phi^{n+1}-\frac{2 \operatorname{Re}}{\Delta t} \Phi^{n+1}=-3 \operatorname{Re} h_{v}^{n}+\operatorname{Re} h_{v}^{n-1}-\nabla^{2} \Phi^{n}-\frac{2 \operatorname{Re}}{\Delta t} \Phi^{n} \\
\Phi_{p}=0 \text { at } \mathrm{y}= \pm 1 \\
\nabla^{2} v_{p}{ }^{n+1}=\Phi_{p}{ }^{n+1} \\
v_{p}=0 \text { at } \mathrm{y}= \pm 1 \\
\nabla^{2} \Phi_{1}{ }^{n+1}-\frac{2 \operatorname{Re}}{\Delta t} \Phi_{1}{ }^{n+1}=0 \\
\Phi_{1}=0 \text { at } \mathrm{y}=1 \\
\Phi_{1}=1 \text { at } \mathrm{y}=-1 \\
\nabla^{2} v_{1}^{n+1}=\Phi_{1}{ }^{n+1} \\
v_{1}=0 \text { at } \mathrm{y}= \pm 1 \\
\nabla^{2} \Phi_{2}{ }^{n+1}-\frac{2 \mathrm{Re}}{\Delta t} \Phi_{2}{ }^{n+1}=0 \\
\Phi_{2}=1 \text { at } \mathrm{y}=1 \\
\Phi_{2}=0 \text { at } \mathrm{y}=-1 \\
\nabla^{2} v_{2}{ }^{n+1}=\Phi_{2}{ }^{n+1} \\
v_{2}=0 \text { at } \mathrm{y}= \pm 1
\end{gathered}
$$

Second order accuracy is obtained by these temporal discretizations.

\subsubsection{Spatial Discretization}

The grid points in the $\mathrm{x}$ and $\mathrm{z}$ directions are defined as:

$$
x_{i}=\frac{i L_{x}}{N_{x}}, \quad i=0,1, \ldots, N_{x}-1
$$




$$
z_{k}=\frac{k L_{z}}{N_{z}}, \quad k=0,1, \ldots, N_{z}-1
$$

The grid points in y direction are defined as Chebyshev-Gauss-Lobatto points:

$$
y_{j}=\cos \left(\frac{\pi j}{N_{y}}\right), \quad j=0,1, \ldots, N_{y}
$$

The whole set is solved in Fourier-Chebyshev space. We denote the Fourier coefficients of $\theta$ as $\hat{\theta}$ and the Chebyshev coefficients as $\tilde{\theta}$. Second derivative with respect to y is denoted as $D^{2}$. The equations of $\theta$ become:

$$
\begin{gathered}
D^{2} \widehat{\widetilde{\theta}}^{n+1}-\left(\alpha^{2}+\beta^{2}\right) \widehat{\widetilde{\theta}}^{n+1}-\frac{2 \operatorname{Re} \operatorname{Pr}}{\Delta t} \widehat{\widetilde{\theta}}^{n+1}=R H S_{T} \\
R H S_{T}=-3 \operatorname{Re} \operatorname{Pr} \widehat{\widetilde{h}}_{T}^{n}+\operatorname{Re} \operatorname{Pr} \widehat{\widetilde{h}}_{T}^{n-1}-D^{2} \widehat{\widetilde{\theta}}^{n}+\left(\alpha^{2}+\beta^{2}\right) \widehat{\widetilde{\theta}}^{n}-\frac{2 \operatorname{Re} \operatorname{Pr}}{\Delta t} \widehat{\widetilde{\theta}}^{n} \\
\nabla^{2} \widehat{\widetilde{\theta}}^{n+1}-\frac{2 \operatorname{Re} \operatorname{Pr}}{\Delta t} \widehat{\widetilde{\theta}}^{n+1}=R H S_{T} \\
R H S_{T}=-3 \operatorname{Re} \operatorname{Pr} \hat{\widetilde{h}}_{T}^{n}+\operatorname{Re} \operatorname{Pr} \widehat{\widetilde{h}}_{T}^{n-1}-D^{2} \widehat{\widetilde{\theta}}^{n}+\left(\alpha^{2}+\beta^{2}\right) \widehat{\widetilde{\theta}}^{n}-\frac{2 \operatorname{Re} \operatorname{Pr}}{\Delta t} \widehat{\widetilde{\theta}}^{n}-2 B r\left(\frac{\widehat{d u}}{d y}\right)^{2}
\end{gathered}
$$

and the boundary conditions are:

$$
\begin{gathered}
\hat{\tilde{\theta}}=\hat{\tilde{\theta}}_{1} \text { at } \mathrm{y}=1 \\
\hat{\tilde{\theta}}=\hat{\tilde{\theta}}_{2} \text { at } \mathrm{y}=-1
\end{gathered}
$$

$\alpha$ and $\beta$ are the wave numbers in $\mathrm{x}$ and $\mathrm{z}$ directions, respectively. Also another parameter $\kappa$ is defined as: 


$$
\kappa=\alpha^{2}+\beta^{2}
$$

and both of the energy equations become:

$$
D^{2} \hat{\tilde{\theta}}^{n+1}-\left(\kappa^{2}+\frac{2 \operatorname{Re} \operatorname{Pr}}{\Delta t}\right) \hat{\tilde{\theta}}^{n+1}=R H S_{T}^{n, n-1}
$$

The equation above is a second order equation in Fourier-Chebyshev space that has to be solved with respect to each $\alpha$ and $\beta$.

For the other equations, exactly the same procedure we followed for $\theta$ should be followed. Therefore, they become:

$$
\begin{gathered}
D^{2} \widehat{\widetilde{G}}^{n+1}-\left(\kappa^{2}+\frac{2 \mathrm{Re}}{\Delta t}\right) \widehat{\widetilde{G}}^{n+1}=R H S_{g}^{n, n-1} \\
\hat{\widetilde{G}}=0 \text { at } \mathrm{y}= \pm 1 \\
R H S_{g}=-3 \operatorname{Re} \hat{\tilde{h}}_{g}^{n}+\operatorname{Re} \hat{\tilde{h}}_{g}^{n-1}-D^{2} \widehat{\widetilde{G}}^{n}+\left(\alpha^{2}+\beta^{2}\right) \widehat{\widetilde{G}}^{n}-\frac{2 \operatorname{Re} \widehat{\widetilde{G}}^{n}}{\Delta t} \\
D^{2} \widehat{\widetilde{\Phi}}_{p}^{n+1}-\left(\kappa^{2}+\frac{2 \operatorname{Re}}{\Delta t}\right) \widehat{\widetilde{\Phi}}_{p}^{n+1}=R H S_{v}^{n, n-1} \\
\widehat{\widetilde{\Phi}}_{p}=0 \text { at } \mathrm{y}= \pm 1 \\
R H S_{g}=-3 \operatorname{Re} \hat{\tilde{h}}_{g}^{n}+\operatorname{Re} \hat{\tilde{h}}_{g}^{n-1}-D^{2} \widehat{\widetilde{\Phi}}_{p}^{n}+\left(\alpha^{2}+\beta^{2}\right) \widehat{\widetilde{\Phi}}_{p}^{n}-\frac{2 \operatorname{Re}}{\Delta t} \widehat{\widetilde{\Phi}}_{p}^{n} \\
D^{2} \hat{\tilde{v}}_{p}^{n+1}-\kappa^{2} \hat{\tilde{v}}_{p}^{n+1}=\widehat{\widetilde{\Phi}}_{p}^{n+1} \\
\hat{\tilde{v}}_{p}^{n+1}=0 \text { at } \mathrm{y}= \pm 1
\end{gathered}
$$




$$
\begin{gathered}
D^{2} \widehat{\widetilde{\Phi}}_{1}^{n+1}-\left(\kappa^{2}+\frac{2 \mathrm{Re}}{\Delta t}\right) \widehat{\widetilde{\Phi}}_{1}^{n+1}=0 \\
\widehat{\widetilde{\Phi}}_{1}=0 \text { at } \mathrm{y}=1 \\
\widehat{\widetilde{\Phi}}_{1}=1 \text { at } \mathrm{y}=-1 \\
D^{2} \hat{\tilde{v}}_{1}^{n+1}-\kappa^{2} \hat{\tilde{v}}_{1}^{n+1}=\widehat{\widetilde{\Phi}}_{1}^{n+1} \\
\hat{\tilde{v}}_{1}^{n+1}=0 \text { at } \mathrm{y}= \pm 1 \\
D^{2} \widehat{\widetilde{\Phi}}_{2}^{n+1}-\left(\kappa^{2}+\frac{2 \mathrm{Re}}{\Delta t}\right) \widehat{\widetilde{\Phi}}_{2}^{n+1}=0 \\
\widehat{\widetilde{\Phi}}_{2}=0 \text { at } \mathrm{y}=1 \\
\widehat{\widetilde{\Phi}}_{2}=1 \text { at } \mathrm{y}=-1 \\
D^{2} \hat{\tilde{v}}_{2}^{n+1}-\kappa^{2} \hat{\tilde{v}}_{2}^{n+1}=\widehat{\widetilde{\Phi}}_{2}^{n+1} \\
\hat{\tilde{v}}_{2}^{n+1}=0 \text { at } \mathrm{y}= \pm 1
\end{gathered}
$$

The transformations between physical space and Fourier-Chebyshev space is done by the help of a real Fast Fourier Transform (FFT). Using FFT causes no problem in Chebyshev space because Gauss-Lobatto points are chosen in ydirection, which leads the discrete Chebyshev expansion become a simple cosine series.

FFT used in this work is a real transform. The complex coefficients of a function in Fourier space are split into two real sets of data and solved separately.

\subsection{Spectral Integration of N-S}

The equations derived above are second order differential equations in FourierChebyshev space, which can also be written in generic form that is second order equation with two boundary conditions:

$$
\theta^{\prime \prime}-\delta \theta=f(y) \text { where } y \in[-1,1]
$$




$$
\begin{gathered}
\theta=\theta_{1} \text { at } y=-1 \\
\theta=\theta_{2} \text { at } y=1
\end{gathered}
$$

Using a Chebyshev-Tau method, we want the solution of:

$$
\theta_{N_{y}}(y)=\sum_{j=0}^{N_{y}} a_{j} T_{j}(y)
$$

with boundary conditions

$$
\begin{gathered}
\sum_{j=0}^{N_{y}}(-1)^{j} a_{j}=\theta_{1} \\
\sum_{j=0}^{N_{y}} a_{j}=\theta_{2}
\end{gathered}
$$

Generally, the system is solved directly for $\theta$ by constructing a spectral differentiation matrix, using the recurrence relations of Chebyshev differentiation. Since, the matrix is ill-conditioned, it has to be reformed. An integral equation had been constructed by solving $\theta^{\prime \prime}(y)$ rather than $\theta$ itself. This approach had been investigated by Greengard [44] and proven to be more accurate than the spectral differentiation method as the number of points increases.

Define:

$$
\theta^{\prime \prime}=\sigma(y)
$$

$\sigma(y)$ and $f(y)$ represented by truncated Chebyshev polynomial expansions it is obtained that: 


$$
\begin{gathered}
\sigma(y)=\sum_{j=0}^{N_{y}} b_{j} T_{j}(y) \\
f(y)=\sum_{j=0}^{N_{y}} f_{j} T_{j}(y)
\end{gathered}
$$

Our original equation becomes:

$$
\begin{gathered}
\sigma(y)-\left[\delta \int_{-1-1}^{y} \int_{-1}^{t} \sigma(\tau) d \tau d t+\delta C_{1} y+\delta C_{0}\right]=f(y) \\
\sum_{j=2}^{N_{y}} a_{j} T_{j}(y)=\int_{-1}^{y} \int_{-1}^{t} \sigma(\tau) d \tau d t \\
a_{0} T_{0}(y)=C_{0} \\
a_{1} T_{1}(y)=C_{1} y
\end{gathered}
$$

Coefficients $\left\{a_{j}\right\},\left\{b_{j}\right\}$ and $\left\{d_{j}\right\}$ represent $\theta, \theta^{\prime \prime}$ and $\theta^{\prime}$ respectively. Finally, $\left\{c_{j}\right\}$ represents constants, namely, $c_{0}=2, c_{1}=1$ for $j>0, c_{j}=0$ for $j>N_{y}$.

The recurrence relation for Chebyshev first integration is:

$$
d_{j}=\frac{1}{2 j}\left(c_{j-1} b_{j-1}-c_{j+1} b_{j+1}\right)
$$

and second integration:

$$
a_{j}=\frac{1}{2 j}\left(c_{j-1} d_{j-1}-c_{j+1} d_{j+1}\right)
$$

The help of Eq. (3.123) and (3.124) gives us the following relation: 


$$
a_{j}=\frac{1}{2 j}\left[\frac{c_{j-1}}{2(j-1)}\left(c_{j-2} b_{j-2}-c_{j} b_{j}\right)-\frac{c_{j+1}}{2(j+1)}\left(c_{j} b_{j}-c_{j+2} b_{j+2}\right)\right]
$$

Using equation (3.119) we obtain:

$$
\begin{gathered}
b_{j}-\frac{\delta}{2 j}\left[\frac{c_{j-1} c_{j-2}}{2(j-1)} b_{j-2}-\frac{c_{j}}{2}\left(\frac{C_{j-1}}{j-1}+\frac{C_{j+1}}{j+1}\right) b_{j}+\frac{C_{j+1} C_{j+2}}{2(j+1)} b_{j+2}\right]=f_{i} \\
-\delta \frac{1}{4 j} \frac{c_{j-1} C_{j-2}}{(j-1)} b_{j-2}+\left[1+\frac{c_{j} \delta}{4 j}\left(\frac{c_{j-1}}{j-1}+\frac{c_{j+1}}{j+1}\right)\right] b_{j}-\delta \frac{C_{j+1} C_{j+2}}{4 j(j+1)} b_{j+2}=f_{i} \\
j=2,3, \ldots, N_{y} \\
p_{j}=-\delta \frac{C_{j-1} C_{j-2}}{4 j(j-1)} \\
q_{j}=1+\frac{\delta c_{j}}{4 j}\left(\frac{c_{j-1}}{j-1}+\frac{c_{j+1}}{j+1}\right) \\
r_{j}=-\delta \frac{c_{j+1} C_{j+2}}{4 j(j+1)}
\end{gathered}
$$

the general form of the equations becomes:

$$
p_{j} b_{j-2}+q_{j} b_{j}+r_{j} b_{j+2}=f_{j}, \quad j=2,3, \ldots, N_{y}
$$

by considering the boundary conditions defined by Eqn. 3.114 and Eqn. 3.115 they can be rewritten

$$
\begin{gathered}
\sum_{j=2}^{N_{\mathrm{y}}}(-1)^{j} a_{j}-C_{1}+C_{0}=\theta_{1} \text { at } \mathrm{y}=-1 \\
\sum_{j=2}^{N_{y}} a_{j}+C_{1}+C_{0}=\theta_{2} \text { at } \mathrm{y}=1
\end{gathered}
$$


also, can be written as

$$
\begin{gathered}
C_{0}+\sum_{j=2}^{N_{y}} a_{j}=\frac{\theta_{1}+\theta_{2}}{2} \text { (even) } \\
C_{1}+\sum_{j=2}^{N_{y}-1} a_{j}=\frac{\theta_{2}-\theta_{1}}{2} \text { (odd) }
\end{gathered}
$$

by using the relation (3.125) for replacing $a_{j}$ in the boundary conditions above with $b_{j}$ we have

$$
\begin{aligned}
& C_{0}+\sum_{j=2}^{N_{y}} \frac{1}{2 j}\left[\frac{c_{j-1}}{2(j-1)}\left(c_{j-2} b_{j-2}-c_{j} b_{j}\right)-\frac{c_{j+1}}{2(j+1)}\left(c_{j} b_{j}-c_{j+2} b_{j+2}\right)\right] \\
& =\frac{\theta_{1}+\theta_{2}}{2} \text { (even) } \\
& C_{1}+\sum_{j=2}^{N_{y}} \frac{1}{2 j}\left[\frac{c_{j-1}}{2(j-1)}\left(c_{j-2} b_{j-2}-c_{j} b_{j}\right)-\frac{c_{j+1}}{2(j+1)}\left(c_{j} b_{j}-c_{j+2} b_{j+2}\right)\right] \\
& =\frac{\theta_{2}-\theta_{1}}{2} \text { (odd) }
\end{aligned}
$$

With these two more boundary conditions which form the top row of the matrices we get the even system

$$
\left[\begin{array}{ccccccc}
1 & t_{2} & t_{4} & t_{6} & \ldots & \ldots & t_{N_{y}} \\
p_{0} & q_{0} & r_{0} & 0 & \ldots & \ldots & 0 \\
0 & p_{2} & q_{2} & r_{2} & 0 & \ldots & 0 \\
\vdots & 0 & \ddots & \ddots & \ddots & 0 & \vdots \\
\vdots & \vdots & 0 & \ddots & \ddots & \ddots & \vdots \\
\vdots & \vdots & \vdots & 0 & p_{N_{y}-2} & q_{N_{y}-2} & r_{N_{y}-2} \\
0 & \ldots & \ldots & \ldots & 0 & p_{N_{y}} & q_{N_{y}}
\end{array}\right]\left[\begin{array}{c}
c_{o} \\
b_{o} \\
b_{2} \\
\vdots \\
b_{j} \\
\vdots \\
b_{N_{y}}
\end{array}\right]=\left[\begin{array}{c}
\frac{\theta_{1}+\theta_{2}}{2} \\
f_{o} \\
f_{2} \\
\vdots \\
f_{j} \\
\vdots \\
f_{N_{y}}
\end{array}\right]
$$


and the odd system

$$
\left[\begin{array}{ccccccc}
1 & t_{1} & t_{3} & t_{5} & \ldots & \ldots & t_{N_{y}-1} \\
p_{1} & q_{1} & r_{1} & 0 & \ldots & \ldots & 0 \\
0 & p_{3} & q_{3} & r_{3} & 0 & \ldots & 0 \\
\vdots & 0 & \ddots & \ddots & \ddots & 0 & \vdots \\
\vdots & \vdots & 0 & \ddots & \ddots & \ddots & \vdots \\
\vdots & \vdots & \vdots & 0 & p_{N_{y}-3} & q_{N_{y}-3} & r_{N_{y}-3} \\
0 & \ldots & \ldots & \ldots & 0 & p_{N_{y}-1} & q_{N_{y}-1}
\end{array}\right]\left[\begin{array}{c}
c_{1} \\
b_{1} \\
b_{3} \\
\vdots \\
b_{j} \\
\vdots \\
b_{N_{y}-1}
\end{array}\right]=\left[\begin{array}{c}
\frac{\theta_{2}-\theta_{1}}{2} \\
f_{1} \\
f_{3} \\
\vdots \\
f_{j} \\
\vdots \\
f_{N_{y}-1}
\end{array}\right]
$$

An important point here is that the cases of $j=0$ and $j=1$ are not included in definitions for $p_{j}, q_{j}$ and $r_{j}$. This problem can be solved by comparing equation (3.121) and the matrices above. In addition to that, $t_{j}$ can be found by Eqns. (3.138) and (3.139). $p_{j}, q_{j}$ and $r_{j}$ are defined in Eqns. (3.128) to (3.130). For $j=0$ and $j=1$ cases:

$$
\begin{aligned}
& b_{o}-\delta C_{o}=f_{o} \\
& b_{1}-\delta C_{1}=f_{1}
\end{aligned}
$$

So

$$
\begin{array}{rll}
p_{o}=-\delta, & \mathrm{q}_{o}=1, & \mathrm{r}_{o}=0 \\
p_{1}=-\delta, & \mathrm{q}_{1}=1, & \mathrm{r}_{1}=0
\end{array}
$$

Using spectral integration method the system formed has $N_{y}+3$ equations and $N_{y}+3$ unknowns which contains $b_{j}$ and two constants $C_{o}$ and $C_{1}$. As seen from above the whole system is split into odd and even matrices where we know the right-hand side function. The matrices are tridiagonal except the top row which is derived from the boundary conditions shown above. First the system is solved 
for $b_{j}$. These are actually the coefficients of $\theta^{\prime \prime}$. Then the Chebyshev integration is carried out twice over $b_{j}$ to get $a_{j}$, which are actually the coefficient of $\theta$. $C_{o}$ and $C_{1}$, which are the integration constants, had been determined in the matrix solver. They are the zeroth and first coefficients of $\theta$ meaning actually $a_{o}$ and $a_{1}$.

\subsection{Computational Procedure}

Here we will give brief information about N-S solver. Before the beginning of each and every time step, we have the data from previous time step, namely

$$
\begin{array}{ccc}
p_{o}=-\delta, & \mathrm{q}_{o}=1, & \mathrm{r}_{o}=0 \\
p_{1}=-\delta, & \mathrm{q}_{1}=1, & \mathrm{r}_{1}=0
\end{array}
$$

All the data above are in Fourier space. All mathematical expressions are done in physical space so with the help of inverse FFT we convert all the initial values to physical space and calculate the non-linear terms as follows

$$
\begin{gathered}
H_{1}=v \omega_{z}-w \omega_{y}-\frac{\partial p}{\partial x} \\
H_{2}=w \omega_{x}-u \omega_{z}+\frac{R a}{\operatorname{Re}^{2} \operatorname{Pr}} \theta \\
H_{3}=u \omega_{y}-v \omega_{x} \\
H_{T}=-\left(u \frac{\partial \theta}{\partial x}+v \frac{\partial \theta}{\partial y}+w \frac{\partial \theta}{\partial z}\right)
\end{gathered}
$$

Transforming back to Fourier space and for each value of $\kappa^{2}=\alpha^{2}+\beta^{2}$ and $\kappa \neq 0$,

$$
\tilde{h}_{g}=i \beta \widetilde{H}_{1}-i \alpha \widetilde{H}_{3}
$$


since

$$
\tilde{h}_{v}=-\frac{\partial}{\partial y}\left(\frac{\partial \widetilde{H}_{1}}{\partial x}+\frac{\partial \widetilde{H}_{3}}{\partial z}\right)+\left(\frac{\partial^{2}}{\partial x^{2}}+\frac{\partial^{2}}{\partial z^{2}}\right) \widetilde{H}_{2}
$$

We transform the results above back to Fourier-Chebyshev space to consolidate the RHS for each equation.

For G-equation:

$$
R H S_{g}=-3 \operatorname{Re} \hat{\tilde{h}}_{g}^{n}+\operatorname{Re} \hat{\tilde{h}}_{g}^{n-1}-D^{2} \hat{\widetilde{G}}^{n}+\left(\kappa^{2}+\frac{2 \operatorname{Re}}{\Delta T}\right) \hat{\widetilde{G}}^{n}
$$

where $\hat{\widetilde{h}}_{g}^{n-1}, D^{2} \widehat{\widetilde{G}}^{n}$ and $\widehat{\widetilde{G}}^{n}$ are obtained from the previous time step.

v-equation: for this special case, the Chebyshev derivative of the first part of $h_{v}$ with respect to y should be taken, and after that obtain $h_{v}$ by adding the second part to it.

$$
R H S_{v}=-3 \operatorname{Re} \hat{\tilde{h}}_{v}^{n}+\operatorname{Re} \hat{\tilde{h}}_{v}^{n-1}-D^{2} \widehat{\widetilde{\Phi}}_{p}^{n}+\left(\kappa^{2}+\frac{2 \operatorname{Re}}{\Delta T}\right) \widehat{\widetilde{\Phi}}_{p}^{n}
$$

where $\hat{\tilde{h}}_{v}^{n-1}, D^{2} \widehat{\widetilde{\Phi}}_{p}^{n}$ and $\widehat{\widetilde{\Phi}}_{p}^{n}$ are obtained from the previous time step.

$\theta$-equation:

$$
R H S_{T}=-3 \operatorname{Re} \operatorname{Pr} \hat{\tilde{h}}_{T}^{n}+\operatorname{Re} \operatorname{Pr} \hat{\tilde{h}}_{T}^{n-1}-D^{2} \hat{\tilde{\theta}}^{n}+\left(\kappa^{2}+\frac{2 \operatorname{Re} \operatorname{Pr}}{\Delta T}\right) \hat{\tilde{\theta}}^{n}
$$

where $\hat{\tilde{h}}_{T}^{n-1}, D^{2} \hat{\tilde{\theta}}^{n}$ and $\hat{\tilde{\theta}}^{n}$ are obtained from the previous time step. 
And at this point, the system can be solved by the vertical solver in FourierChebyshev space. The technique used for this is exactly the method we mentioned in the previous section, namely, spectral integration. Vertical solver gives us the solutions of $\hat{\tilde{u}}, \widehat{\widetilde{G}}, \widehat{\widetilde{G}}^{\prime}, \widehat{\widetilde{f}}, \widehat{\widetilde{\Phi}}, \hat{\tilde{\theta}}, \hat{\tilde{\theta}}^{\prime}$. By the help of these data obtained, we can find the other variables needed for the solution.

We know from continuity

$$
i \alpha \tilde{u}+i \tilde{\beta w}=\tilde{v}^{\prime}
$$

and from the definition of $\mathrm{G}$

$$
i \beta \tilde{u}-i \alpha \tilde{w}=\widetilde{G}
$$

which gives us

$$
\begin{aligned}
& \tilde{u}=\frac{i}{\kappa^{2}}\left(\alpha \tilde{v}^{\prime}-\beta \widetilde{G}\right) \\
& \tilde{w}=\frac{i}{\kappa^{2}}\left(\beta \tilde{v}^{\prime}+\alpha \widetilde{G}\right)
\end{aligned}
$$

also

$$
\begin{aligned}
& \widetilde{\omega}_{x}=\frac{i}{\kappa^{2}}\left(\alpha \widetilde{G}^{\prime}+\beta \widetilde{\Phi}\right) \\
& \widetilde{\omega}_{z}=\frac{i}{\kappa^{2}}\left(\beta \widetilde{G}^{\prime}-\alpha \widetilde{\Phi}\right)
\end{aligned}
$$

from the temperature equation 


$$
\begin{aligned}
& \frac{\partial \tilde{\theta}}{\partial x}=\alpha \tilde{\theta} \\
& \frac{\partial \tilde{\theta}}{\partial z}=\beta \tilde{\theta}
\end{aligned}
$$

Separation of real and imaginary parts of the coefficients in Fourier space is an important point to be taken care of. All the data in the form of Fourier coefficients should be divided into two sets of real data and the calculations should be based on these two sets of data. An example for this can be:

$$
h_{g}=i \beta H_{1}-i \alpha H_{3}
$$

but when we are doing this operation what we really want to calculate is

$$
h_{g}=i \beta\left(H_{1 r}+i H_{1 i m}\right)-i \alpha\left(H_{3 r}+i H_{3 i m}\right)
$$

which is for the negative wave number. For positive wave number the combination is just the conjugate $H_{1 r}-i H_{1 i m}$. Separating the real and imaginary parts we get

$$
\begin{aligned}
& h_{g r}=-\beta H_{1 i m}+\alpha H_{3 i m} \\
& h_{g i m}=i\left(\beta H_{1 r}-\alpha H_{3 r}\right)
\end{aligned}
$$

treating these two data sets separately and calling the same function, vertical solver, in the computer program twice to handle the solution of real and imaginary parts in Fourier-Chebyshev space.

For $\kappa=0$, we can solve the equations directly because for this case everything is real even in Fourier space. One can easily see for this case 


$$
i \beta \tilde{u}-i \alpha \tilde{w}=\widetilde{G}=0
$$

and by the help of continuity equation and the boundary conditions

$$
\tilde{v}=0
$$

Solving the momentum equations in $\mathrm{x}$-direction and $\mathrm{z}$-direction directly

$$
\begin{aligned}
& \frac{\partial \tilde{u}}{\partial t}=\widetilde{H}_{1}+\frac{1}{\operatorname{Re}} D^{2} \tilde{u} \\
& \frac{\partial \tilde{w}}{\partial t}=\widetilde{H}_{3}+\frac{1}{\operatorname{Re}} D^{2} \widetilde{w}
\end{aligned}
$$

Therefore passing through the same temporal discretization procedure, the equations to be solved in Fourier-Chebyshev space is:

$$
\begin{gathered}
D^{2} \hat{\tilde{u}}^{n+1}-\frac{2 \operatorname{Re}}{\Delta T} \hat{\tilde{u}}^{n+1}=-3 \operatorname{Re} \widehat{\widetilde{H}}_{1}^{n}+\operatorname{Re} \widehat{\widetilde{H}}_{1}^{n-1}-D^{2} \hat{\tilde{u}}^{n}-\frac{2 \operatorname{Re}}{\Delta T} \hat{\tilde{u}}^{n} \\
D^{2} \hat{\widetilde{w}}^{n+1}-\frac{2 \operatorname{Re}}{\Delta T} \stackrel{\vec{w}}{w+1}^{n}=-3 \operatorname{Re} \widehat{\widetilde{H}}_{3}^{n}+\operatorname{Re} \widehat{\widetilde{H}}_{3}^{n-1}-D^{2} \hat{\tilde{w}}^{n}-\frac{2 \operatorname{Re}}{\Delta T} \hat{\widetilde{w}}^{n}
\end{gathered}
$$

these equations are for the case $\alpha=0$ and $\beta=0$ which are solved exactly the way described above for $\kappa^{2} \neq 0$ case.

Here at the end of each loop in the code we have ended up having the velocity and temperature fields, which are in Fourier space and dumped into the specified files with their physical values. This is done exactly for each loop so for the next loop we have any information needed for the calculation of RHS of each equation mentioned above. The loop continues until the specific time reached namely $t_{\text {end }}$. 


\section{CHAPTER 4}

\section{RESULTS AND DISCUSSION FOR FORCED CONVECTION IN MICROCHANNELS}

Brinkman number $(\mathrm{Br})$ is introduced in $\mathrm{N}-\mathrm{S}$ solver through the addition of viscous dissipation term widely neglected in conventional channels. Brinkman number is generally neglected for conventional channels in low viscosity and low speed flows for short lengths. However, Brinkman number is significant for long macrochannels. This is due to the fact that; length over diameter ratio is large in microchannels. By making a simple analogy, it is easily seen that Brinkman number may become important for flows in microchannels and may be responsible for the observed unusual behavior between $\mathrm{Nu}$ and $\mathrm{Re}$.

Brinkman number represents the relative importance of viscous heating to fluid conduction and in this study the effect of Brinkman number on single phase heat transfer analysis of hydrodynamically and thermally developed laminar flow of a viscous fluid through horizontal parallel plates is analyzed. The generally neglected viscous dissipation term is inserted in the energy equation. The momentum and energy equation is solved by spectral methods. The top and bottom plates are at prescribed dimensionless temperatures given by Ra. In both $x$ and $z$ directions, velocity components and temperature have periodic boundary conditions.

A horizontal channel that is infinite in streamwise and spanwise directions is considered. The channel height is taken as $3.1 \times 10^{-4} \mathrm{~m}$ which falls within the dimension ranges of microchannels. The selected computational domain represents a section in the middle of a very long and wide microchannel. The 
geometry of the problem considered in this section is shown in Figure 1.1. The coordinate system is located at the center of the parallel plates.

\subsection{Fully Developed Velocity Distribution}

The velocity profile at the upstream boundary is prescribed as a parabolic one and it stays parabolic throughout the length of the channel. Figure 4.1 indicates the velocity profile throughout the channel.

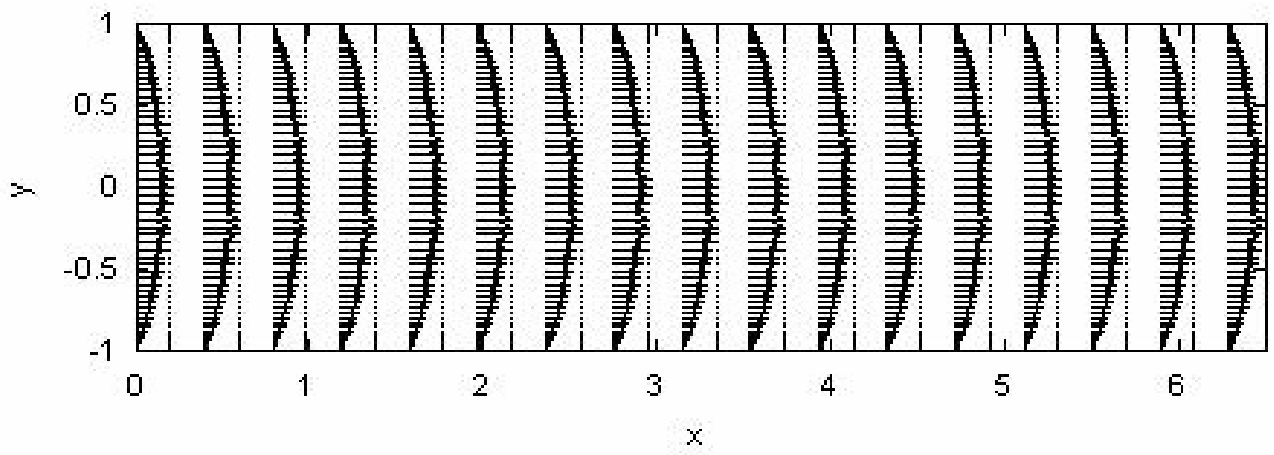

Figure 4.1. Fully developed laminar velocity profile inside a microchannel between parallel plates

In microchannel flows, transition to turbulence occurs much earlier than $\operatorname{Re}=2300$. The selected range of $\operatorname{Re}(80-300)$ corresponds to laminar flow. The plates bounding the channel are also considered as smooth.

\subsection{Heat Transfer Analysis}

For heat transfer analysis the following steps are followed: As a first step to solve the energy equation, Eqns. 1.4 is used for the energy equation with viscous dissipation. Eqn. 1.5 is the simplified version of the viscous dissipation term as it is the form widely accepted in the literature for conventional channels [39]. Eqn. 
1.8 are used as the boundary conditions for the temperature field mentioned in Chapter 1.

By making the energy equation non-dimensional with the help of the equations written in Chapter 3, we ended up with Eqn. 3.9:

$$
\frac{\partial \theta^{*}}{\partial t^{*}}+U^{*} \cdot \nabla \theta^{*}=\frac{1}{\operatorname{Re} \operatorname{Pr}} \nabla^{2} \theta^{*}+\frac{B r}{\operatorname{Re} \operatorname{Pr}}\left(\frac{d u^{*}}{d y^{*}}\right)^{2}
$$

Where Brinkman number is defined as:

$$
B r=\frac{\mu \bar{U}^{2}}{k \Delta T}
$$

After obtaining the temperature profile, the local heat transfer coefficient and $\mathrm{Nu}$ number are found by,

$$
T=\theta^{*}\left(T_{\max }-T_{\min }\right)+T_{m}
$$

where

$$
\theta^{*}=\theta^{*}(y)
$$

and

$$
\begin{gathered}
\Delta T=T_{\max }-T_{\min } \\
\frac{\partial T}{\partial y}=\Delta T \frac{\partial \theta^{*}}{\partial y} \\
\left(\left.\frac{\partial T}{\partial y}\right|_{y=-1}=\Delta T\left(\left.\frac{\partial \theta^{*}}{\partial y}\right|_{y=-1}\right.\right.
\end{gathered}
$$




$$
\left(\frac{\partial T}{\partial y}\right)_{y=-1}=\frac{\Delta T}{L}\left(\left.\frac{\partial \theta^{*}}{\partial y^{*}}\right|_{y=-1}\right.
$$

and $\mathrm{Nu}$ number can be written by utilizing the equations above:

$$
N u=\frac{h L}{k}
$$

where local heat transfer coefficient is found by using:

$$
h_{x}=\frac{k}{\left(T_{m}-T_{w}\right)}\left(\left.\frac{\partial T}{\partial y}\right|_{y=-1}\right.
$$

where $U_{m}$ is given in Table 4.1 and $T_{m}$ is:

$$
T_{m}=\frac{\int_{-1}^{1} T \cdot u \cdot d y}{U_{m} \cdot L}
$$

Introducing dimensionless quantities into the Eqn. 4.7, the Nusselt number is determined for constant wall temperature as:

$$
N u=\left(\frac{\partial \theta^{*}}{\partial y}\right)_{y=-1} \frac{\Delta T}{T_{w}-T_{m}}
$$

\subsubsection{Test cases for numerical experimentation}

The test cases by selecting working fluid as liquid water are shown in Table 4.1. The independent parameters are Re, mean flow velocity, $\mathrm{u}_{\mathrm{m}}$ and channel height, $\mathrm{L}$, which is constant. From the definition of $\operatorname{Re}=\left(u_{m} L\right) / v$, the kinematic viscosity is obtained and and by using a table lookup, bulk temperature is estimated. The 
rest of the columns in Table 4.1 are obtained using this temperature value. As a result $\mathrm{Br}$ values come out as very small but in a similar range with the ranges considered by some other studies in the literature. Since velocity and Re values are very low, natural convection may also be important, thus Ra is also calculated and used.

Therefore, we followed exactly the same procedure with Tso and Mahulikar [10] to obtain the other necessary numbers. They found $v$ and $T_{f}$ is found accordingly. After these critical points all of the other properties of water flowing inside the channel are found by interpolation. However to determine the Ra number, we do need to fix an additional non-dimensional number which is our crucial Brinkman number. By using this non-dimensional number $\Delta T$ is found followed by finding the Ra number.

In this work, three different cases (no-axial conduction, with axial conduction and $\mathrm{Br}$ included in the energy equation) with 12 different sets of data (see Table 4.1), making a total of 36 , are inspected. The reason for choosing these data is due to the lack of experimental data for our parallel plate microchannel numerical experimentation case. We use a parallel logic with Tso and Mahulikar [10] for obtaining the parameters but follow a different way with our geometry and putting the natural convection effect into our work, resulting in mixed convection in parallel plate microchannel.

Our Re ranges over 80-300, which is widely accepted as laminar region in the literature. One of the important parameters, which has smaller values compared quantitatively with macrochannel case numbers, is the value of Ra. The reason for this is the small characteristic length due to the nature of the problem. Increasing $\mathrm{Ra}$ to its macro case values brings our temperature between two walls to impossible to reach values. 
Table 4.1 Selected cases for the simulation runs

\begin{tabular}{|c|c|c|c|c|c|c|c|}
\hline $\boldsymbol{R} \boldsymbol{V}$ & $\boldsymbol{v} \times 10^{7}$ & $\boldsymbol{T}_{\boldsymbol{f}}$ & $\boldsymbol{k}$ & $\boldsymbol{P r}$ & $\Delta \boldsymbol{T}$ & $\boldsymbol{B r} \times \mathbf{1 0}^{5}$ & $\boldsymbol{R a}$ \\
\hline$(--)$ & $\left(\mathrm{m}^{2} \mathrm{~s}^{-1}\right)$ & $(\mathrm{K})$ & $\left(\mathrm{W} \mathrm{m}^{-1} \mathrm{~K}^{-1}\right)$ & $(--)$ & $(\mathrm{K})$ & $(--)$ & $(--)$ \\
\hline Case 1 & \multicolumn{7}{|c|}{$U_{m}=0.25 \mathrm{~m} \mathrm{~s}^{-1} L=3.1 \times 10^{-4} m$} \\
\hline 80 & 9.688 & 294.736 & 0.601 & 6.736 & 3.548 & 2.8333 & 25.219 \\
\hline 87 & 8.908 & 298.576 & 0.607 & 6.129 & 5.048 & 1.8109 & 38.007 \\
\hline 97 & 7.99 & 303.142 & 0.614 & 5.415 & 10.74 & 0.7532 & 87.448 \\
\hline 107 & 7.243 & 308.364 & 0.622 & 4.851 & 15.876 & 0.4559 & 138.72 \\
\hline Case 2 & \multicolumn{7}{|c|}{$U_{m}=0.29 \mathrm{~m} \mathrm{~s}^{-1} L=3.1 \times 10^{-4} m$} \\
\hline 93 & 9.667 & 294.839 & 0.601 & 6.719 & 3.059 & 4.4115 & 21.773 \\
\hline 100 & 8.99 & 298.172 & 0.606 & 6.193 & 3.457 & 3.5956 & 25.859 \\
\hline 116 & 7.75 & 304.818 & 0.617 & 5.234 & 4.363 & 2.4100 & 36.297 \\
\hline 124 & 7.25 & 308.315 & 0.621 & 4.856 & 6.705 & 1.4541 & 58.544 \\
\hline 136 & 6.61 & 312.788 & 0.628 & 4.373 & 7.27 & 1.2091 & 68.008 \\
\hline Case 3 & \multicolumn{7}{|c|}{$U_{m}=0.76 \mathrm{~m} \mathrm{~s}^{-1} L=3.1 \times 10^{-4} \mathrm{~m}$} \\
\hline 250 & 9.424 & 296.034 & 0.603 & 6.531 & 2.303 & 39.1 & 16.676 \\
\hline 273 & 8.63 & 299.946 & 0.609 & 5.913 & 4.049 & 20.133 & 31.176 \\
\hline 300 & 7.853 & 304.096 & 0.616 & 5.312 & 4.936 & 14.853 & 40.678 \\
\hline
\end{tabular}

\subsubsection{Numerical Solution}

The rotational form of Navier-Stokes equations $(\mathrm{N}-\mathrm{S})$ is solved using a spectral methods based approach. Three different cases are considered: i) with axialconduction; ii) without axial-conduction; and iii) with the introduction of $\mathrm{Br}$.

\subsection{Numerical Verifications}

\subsubsection{D vs. 3D}

The Navier-Stokes solver used in this study is originally designed for 3D simulations. However, when the flow is laminar and the bottom plate is heated uniformly, there is no spanwise flow thus the layers of 3-D simulation results were identical leading to absolute 2-D behavior. After observing this behavior, all presented results were obtained from 2-D runs. 

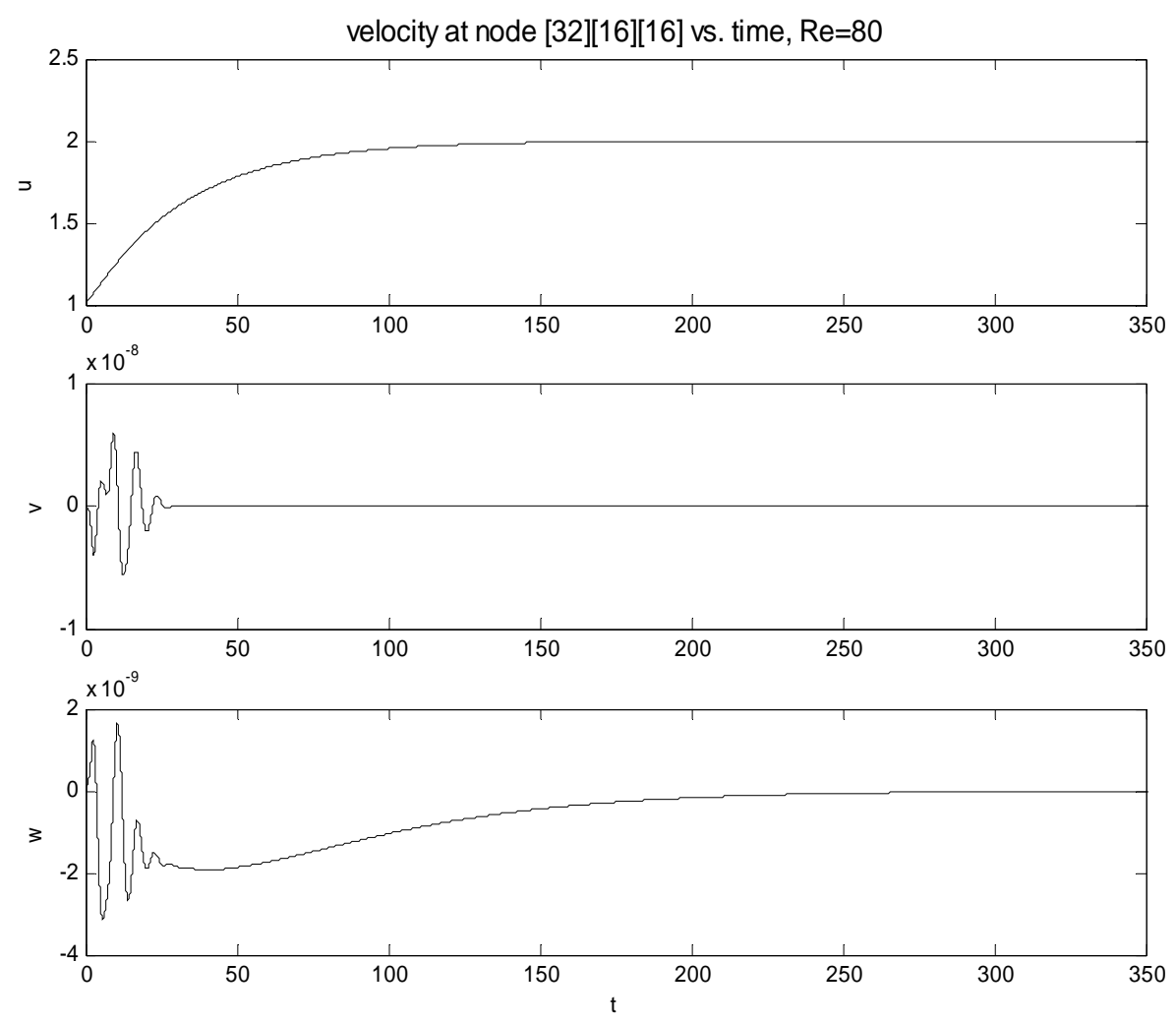

Figure 4.2 $\mathrm{Re}=80$ dimensionless velocities (3D)

For verification of this phenomenon two different runs are made to see that the velocity component in spanwise direction is zero in addition to the same results of $u$ and $v$ for 2D and 3D runs shown in Figures 4.2 and 4.3. The data are taken in the middle of the channel both for $2 \mathrm{D}$ and $3 \mathrm{D}$ runs. It is observed that the results are repeating themselves in all slices in $\mathrm{z}$ direction. 

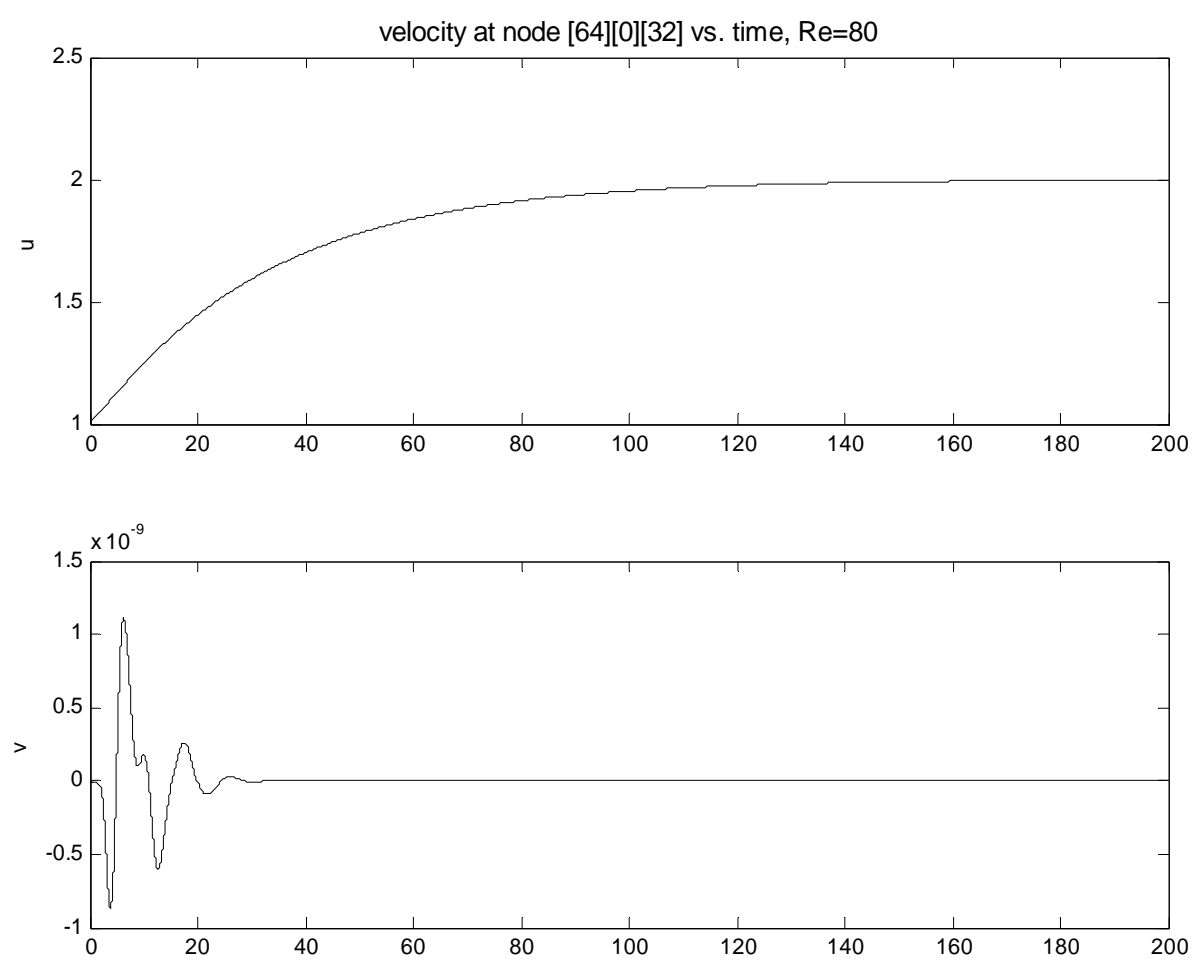

Figure $4.3 \mathrm{Re}=80$ dimensionless velocities (2D)

\subsubsection{Grid Independency}

The only way to establish grid independent solutions is to setup a model with a finer mesh (a larger number of collocation points) and analyze it to see if there are major differences in velocity and temperature fields. In 2-D case 128 and 64 points in $y$ and $x$ directions, respectively, is selected (128x64 grid).

To show the grid independency for $\mathrm{Re}=300$ case, a run with $256 \times 128$ grid was tried and no difference is observed with the coarser grid results. However, for $64 \times 32$ grid, as it is shown in Figure 4.4, it is obvious that the mesh is not suitable for simulating the flow in correct manner. Similar results are also obtained for the temperature fields. Consequently, it is concluded that $128 \times 64$ is the necessary and 
sufficient mesh for the work at hand. Therefore, the Figures 4.5 and 4.6 support the choice made.
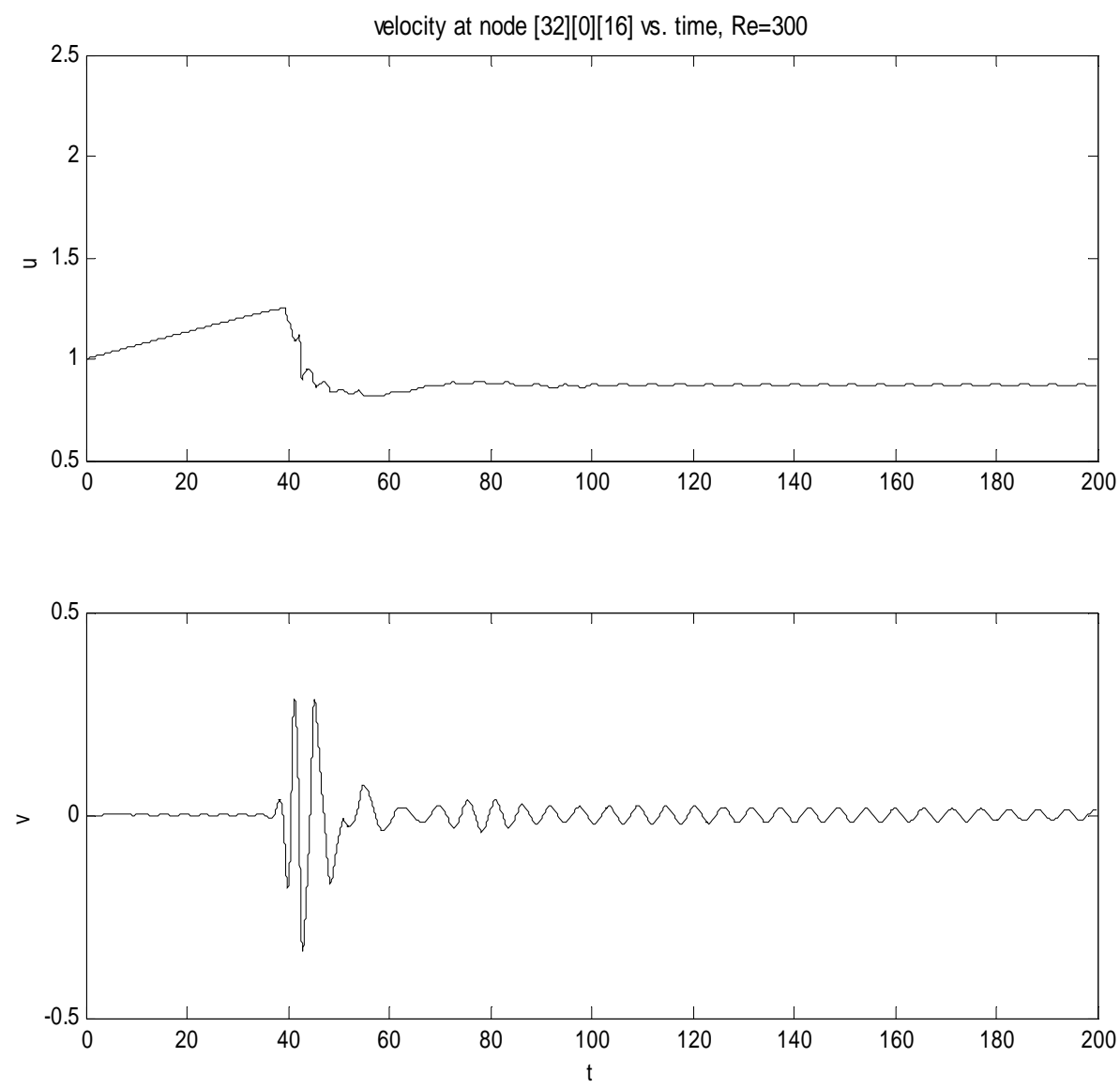

Figure 4.4 $\mathrm{Re}=300$, dimensionless velocities for $64 \times 32$ mesh 

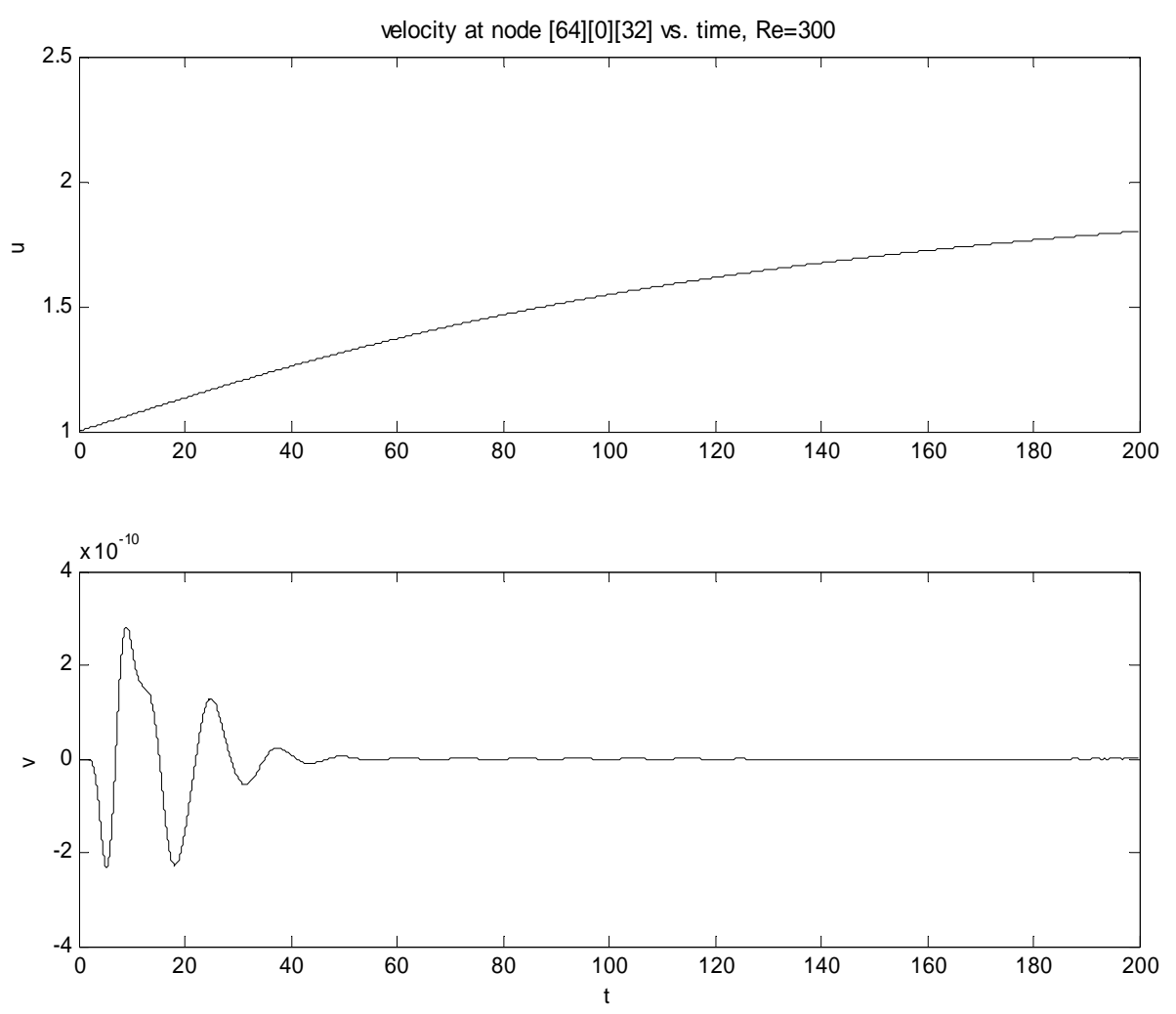

Figure 4.5 $\mathrm{Re}=300$, dimensionless velocities for $128 \times 64$ mesh

The next step followed after determining the mesh is finding the appropriate time scale $d t$. Obtaining the diffusion time scale goes parallel with finding the adequate mesh. We began with $\mathrm{dt}=0.01$ and $64 \times 32$ mesh. After applying same $\mathrm{dt}$ to $128 \times 64$, it is observed that after dimensionless time $\mathrm{t}=101.6$, the results appear as "not a number" at all of the velocity files, which means that this dt cannot catch up a finer mesh, namely $128 \times 64 . \mathrm{dt}=0.005$ is applied for $128 \times 64$ and $\mathrm{dt}=0.0025$ for $256 \times 128$ mesh. Consequently, selecting $128 \times 64$ as our mesh, we automatically determine $\mathrm{dt}=0.005$ as a diffusion time scale. 

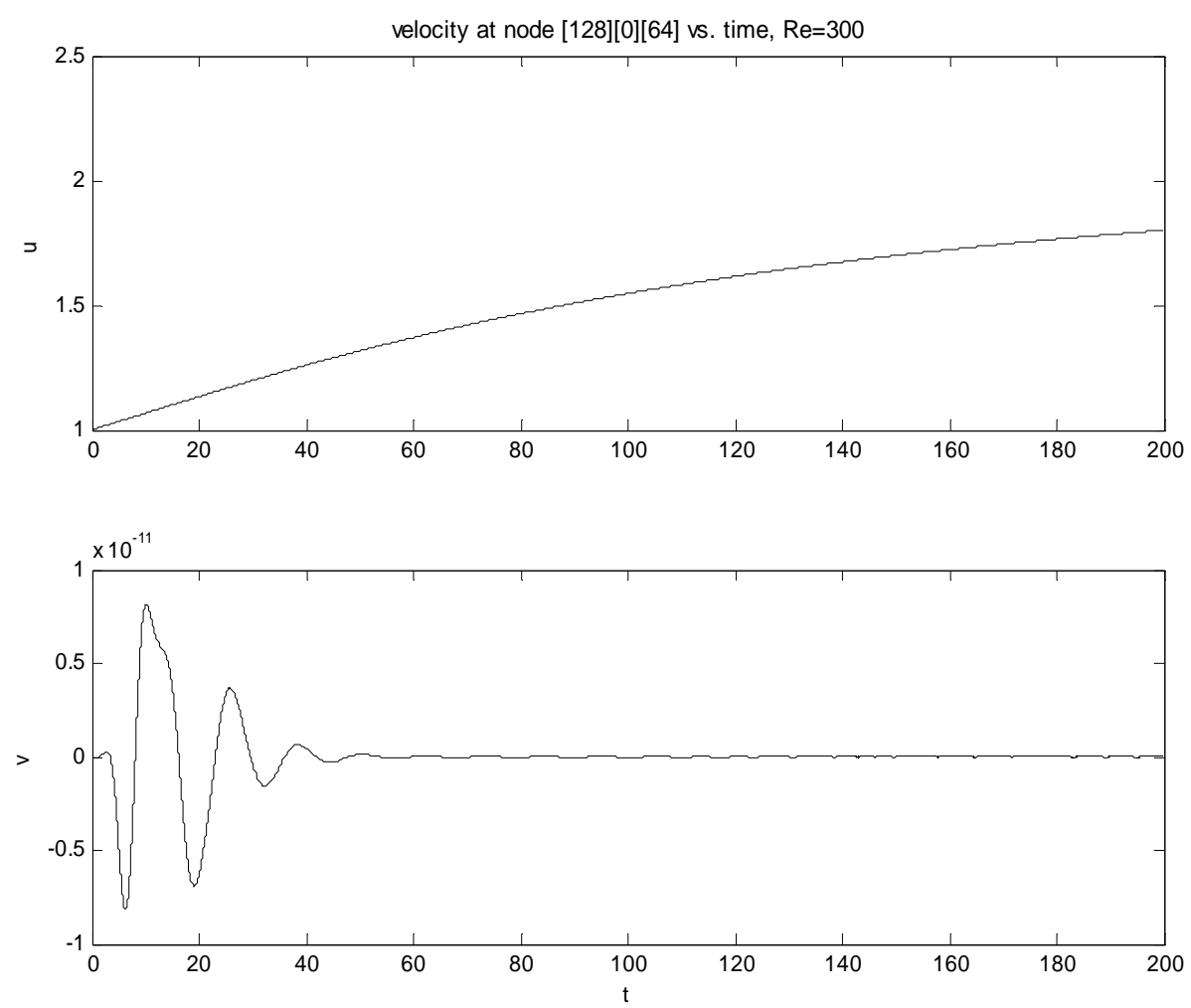

Figure 4.6 $\mathrm{Re}=300$, dimensionless velocities for $256 \times 128$ mesh

\subsection{Results and Discussion}

To show the effects of Brinkman number and axial conduction, the energy equation is solved numerically by spectral methods including the axial conduction and viscous dissipation terms. The dimensionless temperature distribution, streamlines and the $\mathrm{Nu}$ number are determined as a function of dimensionless axial coordinate for different Re, Ra and Brinkman number values. As it could be seen from Table 4.1, the values of Re for the test cases are ranging in 80-300 with corresponding $\mathrm{Br}$ values for the cases with viscous dissipation. The selected Reynolds numbers are adapted from [10] and within the range of laminar regime for microchannels. 
$\mathrm{Br}=0$ is the case without viscous dissipation.

For each Re case in Table 4.1, three different runs: 1) with axial conduction; 2) with no-axial conduction; and 3) with the introduction of $\mathrm{Br}$, are performed. There was no observable difference among the results of these three runs when the results are drawn using contour plots except $\mathrm{Re}=80,87$ and 97 cases.
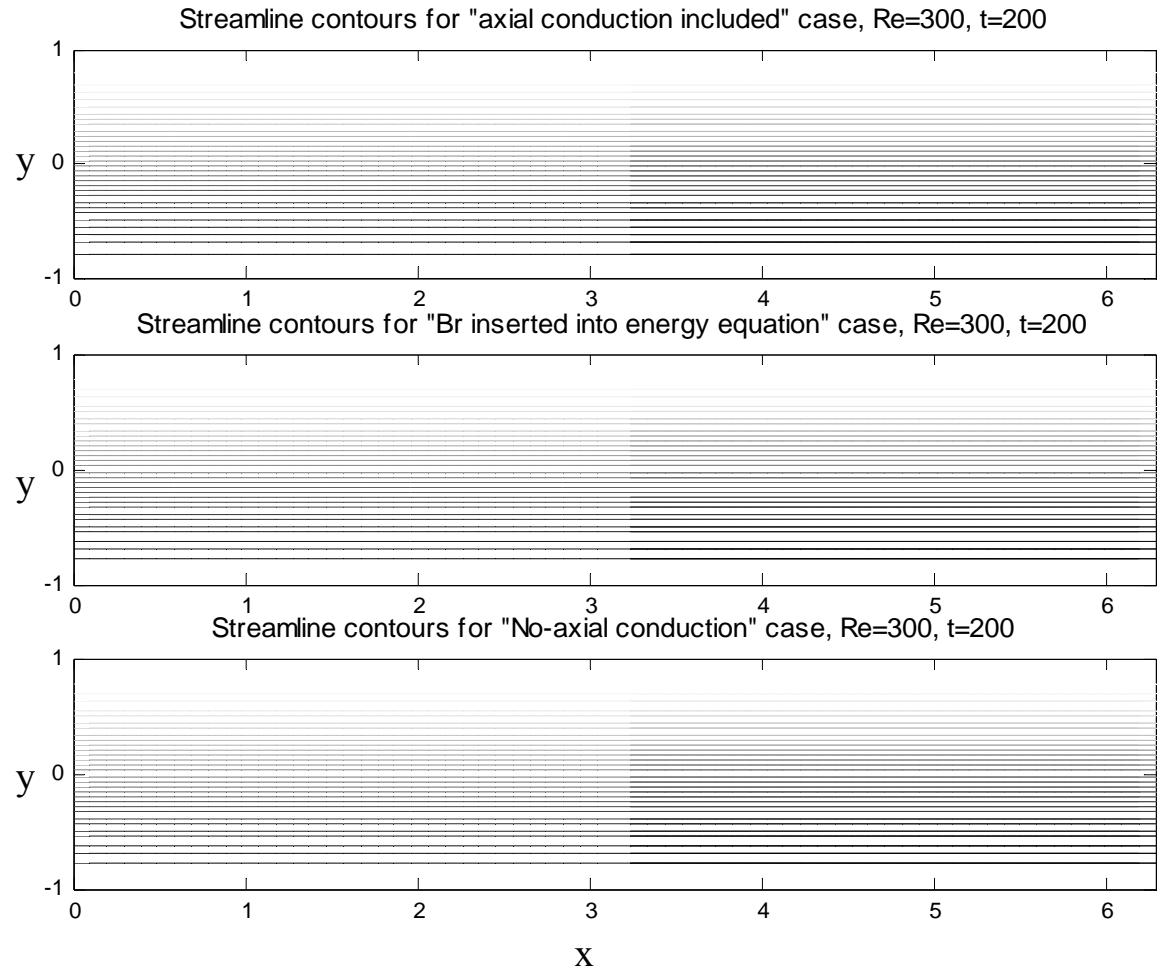

Figure $4.7 \mathrm{Re}=300$ streamline contours at $\mathrm{t}^{*}=200$ with $128 \times 64$ resolution.

As a representative result, Figure 4.7 shows $\mathrm{Re}=300$ streamline results for all three different cases at $t^{*}=200$. It is seen here that the streamlines are straight lines as expected from conventional channels. And the effect of axial conduction and viscous dissipation is not observed from contour plots. Figure 4.8 shows temperature field contours for the same cases. 

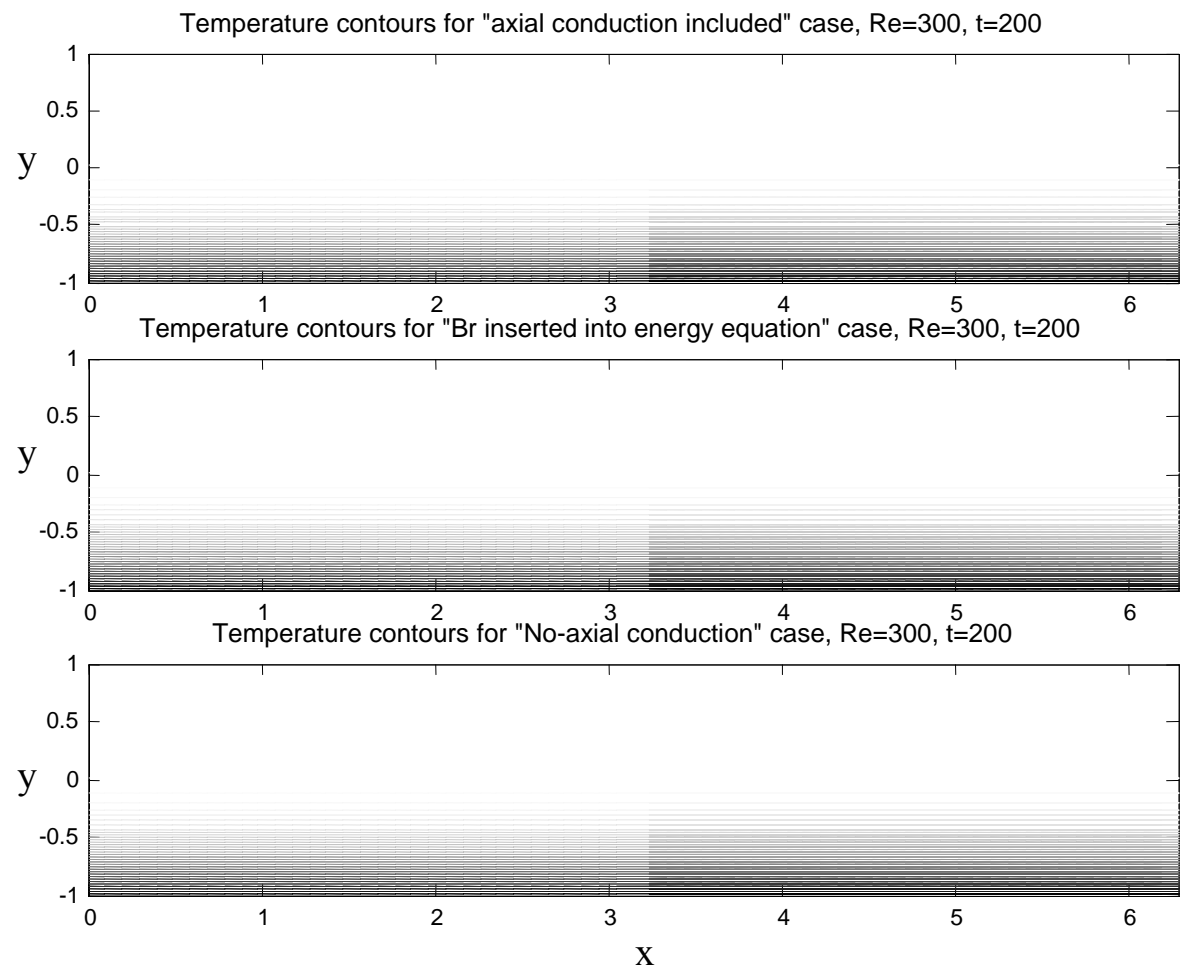

Figure 4.8 $\mathrm{Re}=300$ temperature contours at $\mathrm{t}^{*}=200$ with $128 \times 64$ resolution.

$\mathrm{Re}=80$ case with no-axial conduction gave interesting results. To improve the resolution at that $\mathrm{Re}$, the runs are repeated with $256 \times 128$ collocation points. Figure 4.9 shows comparison of no-axial and $\mathrm{Br}$ streamline results at $\mathrm{t}^{*}=200$. Figure 4.10 is for the same cases, to show temperature fields. Although not as strong, similar behavior is also observed for $\mathrm{Re}=87$ and 97 cases. 

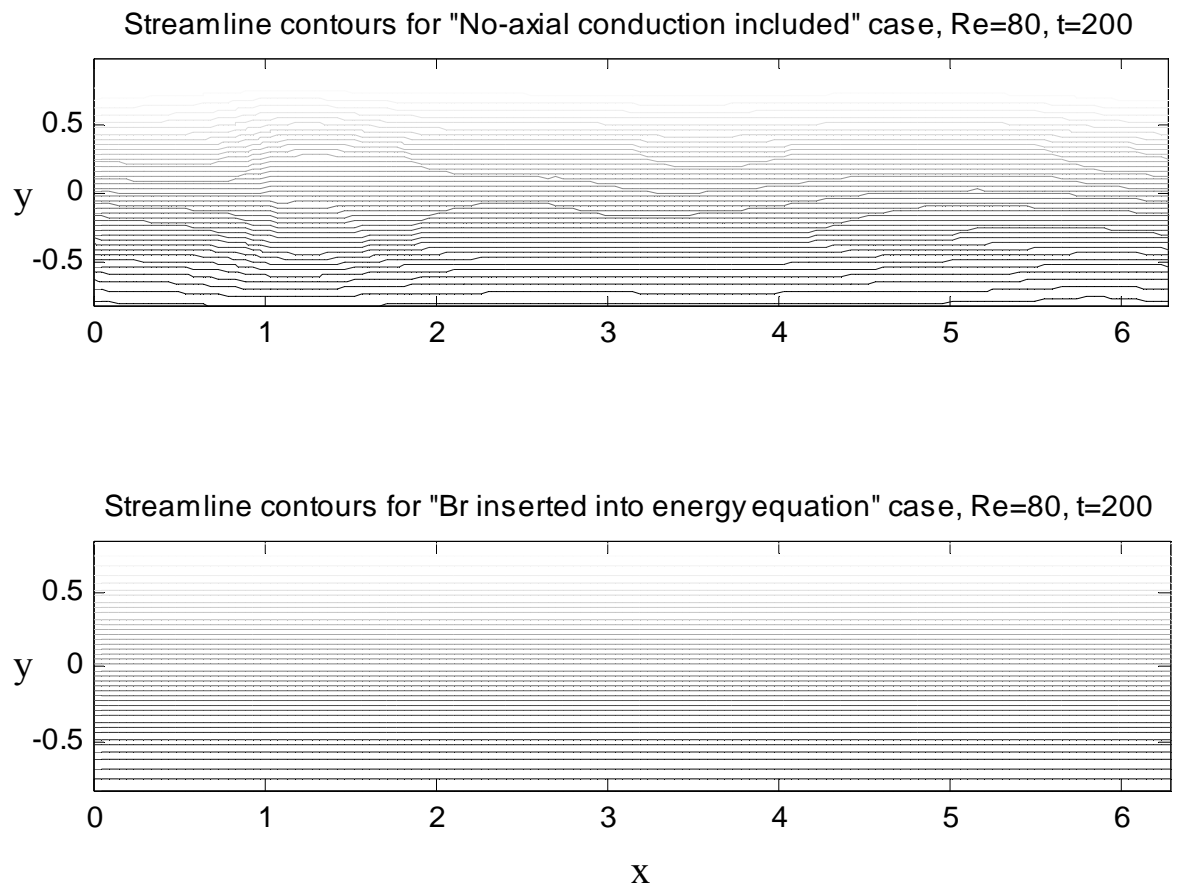

Figure 4.9. Comparison of $\mathrm{Re}=80$ no-axial conduction and $\mathrm{Br}$ (streamline) results at $\mathrm{t}^{*}=200$ with $256 \times 128$ resolution. 

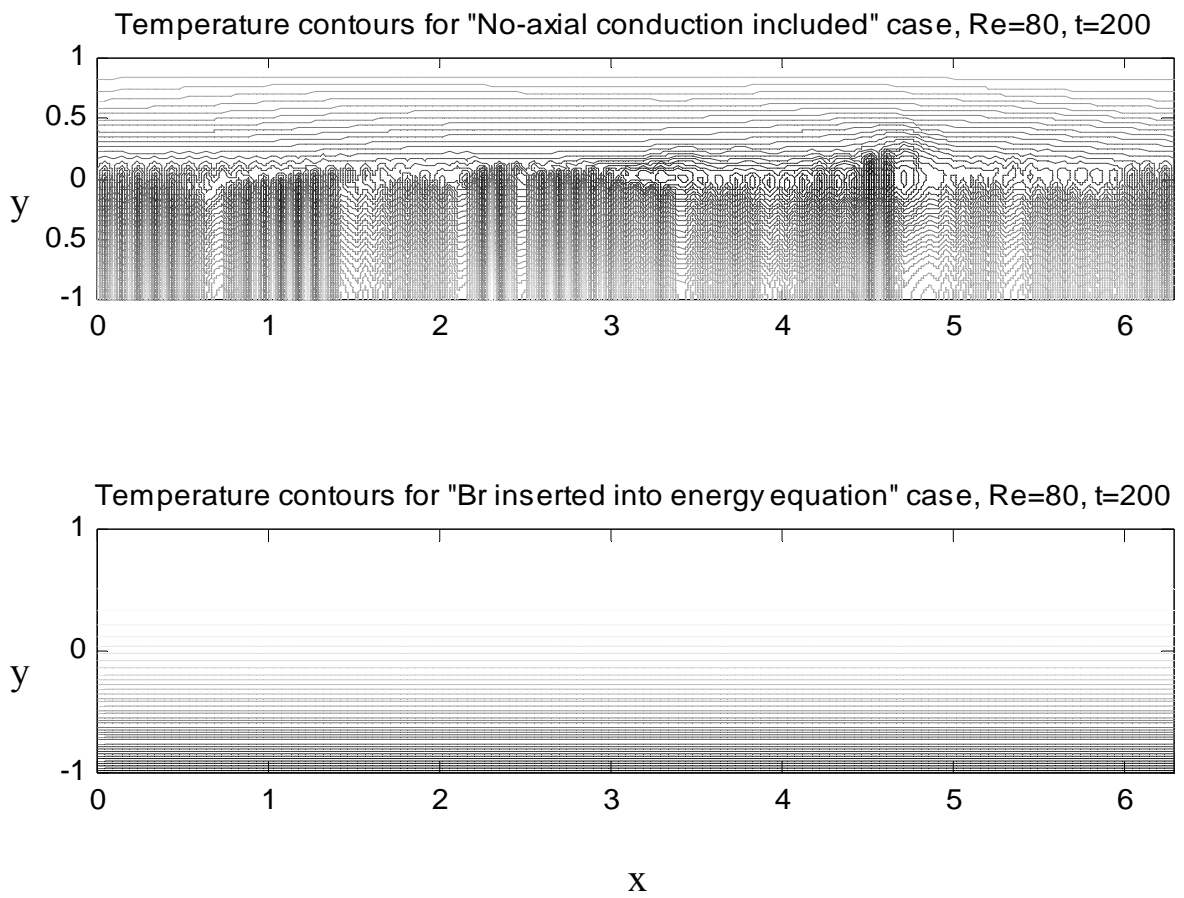

Figure 4.10. Comparison of $\mathrm{Re}=80$ no-axial conduction and $\mathrm{Br}$ temperature contours at $\mathrm{t}^{*}=200$ with $256 \times 128$ resolution.

The streamline contours close to the plates in Figure 4.9 indicates that no-axial conduction case shows a pattern similar to Rayleigh-Benard convection cells appearing due to thermal instability resulting from bottom heated arrangement. The time role of change of $\mathrm{u}$ and $\mathrm{v}$ velocities at the middle of the channel is shown in Figure 4.11. As can be seen, the cell formation starts around $t^{*}=115$. This behavior is most probably due to the increased importance of natural convection after removal of the conduction term from the energy equation. 

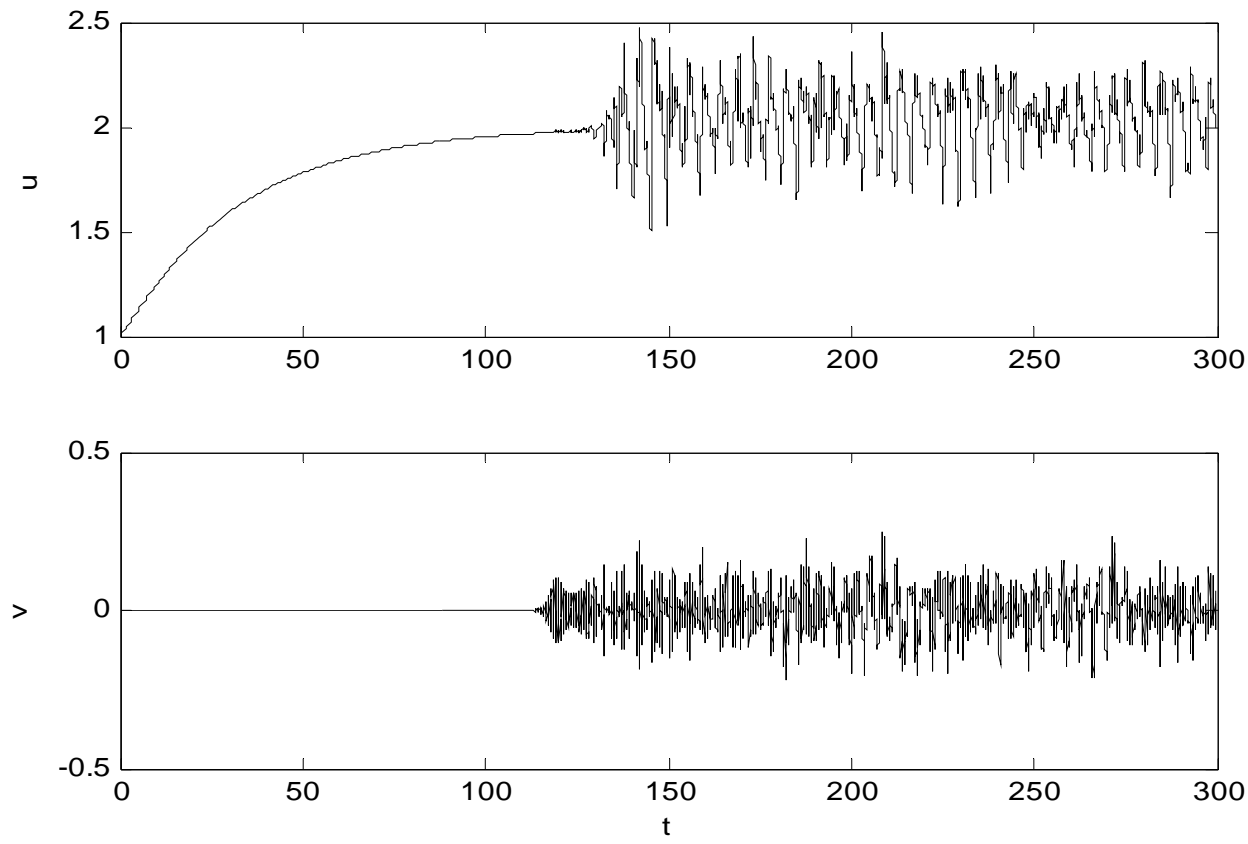

Figure 4.11. Time change of $u$ and $v$ velocities at the middle of the channel for $\mathrm{Re}=80$ no-axial conduction case

For this instability case, the progression of streamline contours over time is shown in Figure 4.12 . As can be seen, at $t^{*}=100$, a very similar result to all other Re case results is obtained. 

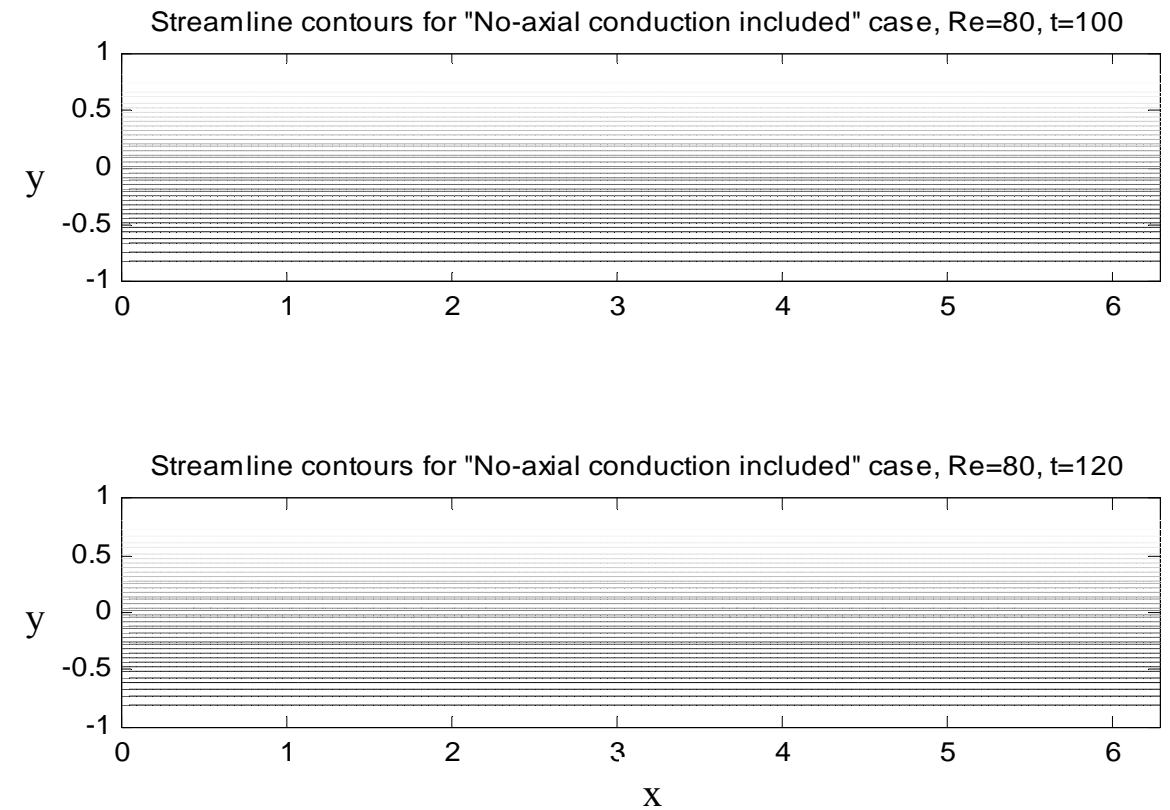

Figure 4.12. For $\mathrm{Re}=80$ no-axial conduction case, streamline contours at

$$
\mathrm{t}^{*}=100,120
$$

At $t^{*}=160$ and 300 , the traces of partially formed convection cells can be seen, Figure 4.13.

Consequently, we can draw an important conclusion for the case of "no-axial conduction included in the energy equation" from Figures 4.12-14. In $\mathrm{Re}=80,87$ and 97 no-axial conduction runs, partially formed convection cells are observed. These structures are not present in the runs with axial conduction and in the runs with $\mathrm{Br}$ for those Reynolds numbers. This unusual behavior shows that axial conduction plays an important role in low Re liquid flows and it should be included in formulations for low Re microchannel simulations. This observation matches the similar conclusions drawn for conventional channels in the literature [32]. 

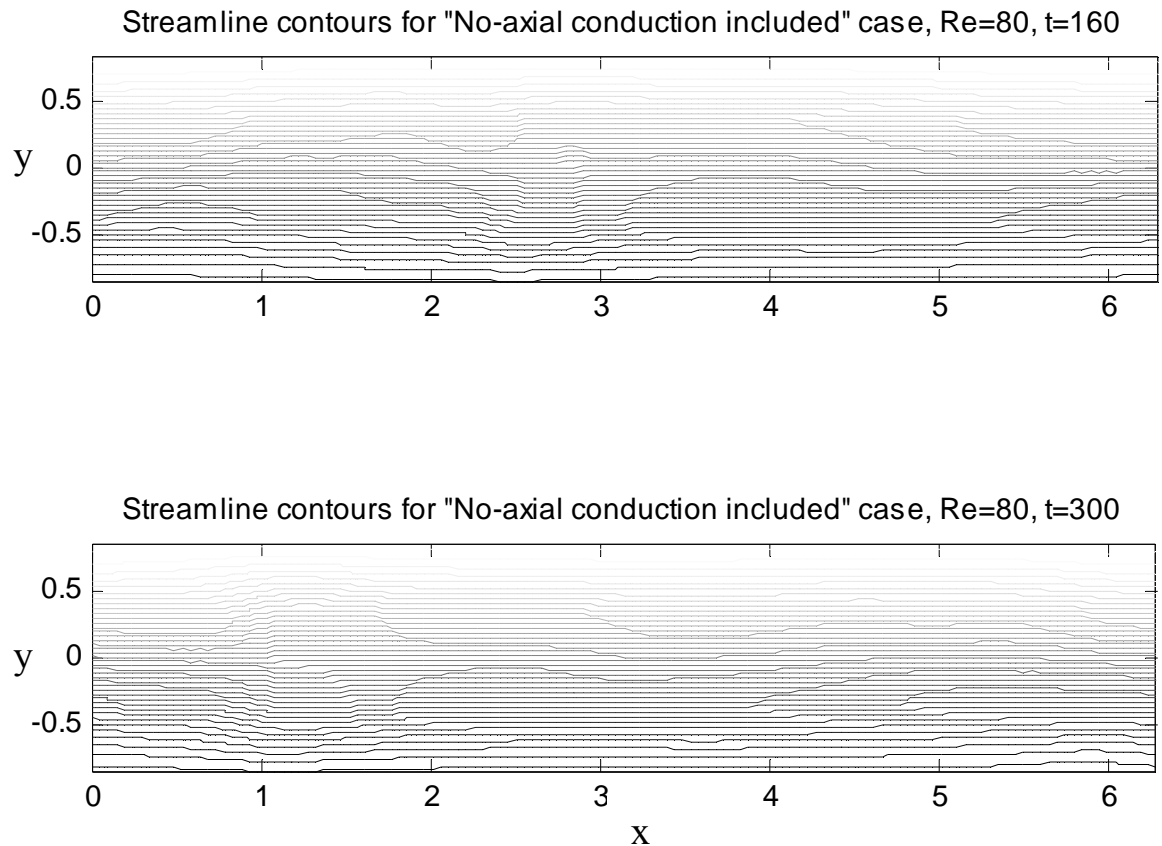

Figure 4.13. For $\mathrm{Re}=80$ no-axial conduction case, streamline contours at

$$
\mathrm{t}^{*}=160,300
$$

The axial conduction term becomes negligible at high Reynolds numbers due to the fact that the Peclet number is sufficiently large so that longitudinal (axial) conduction could be neglected. However, our numerical experiments are performed for relatively small Re (therefore Pe). This is why the we did observe the effect of axial conduction term clearly for lower values (as it is seen from Figure 4.12, $\mathrm{t}^{*}=160$ and 300), but did not see any effect for higher values of Re.

Similar behavior is even better observed in Figures 4.14-15, showing temperature contours. 


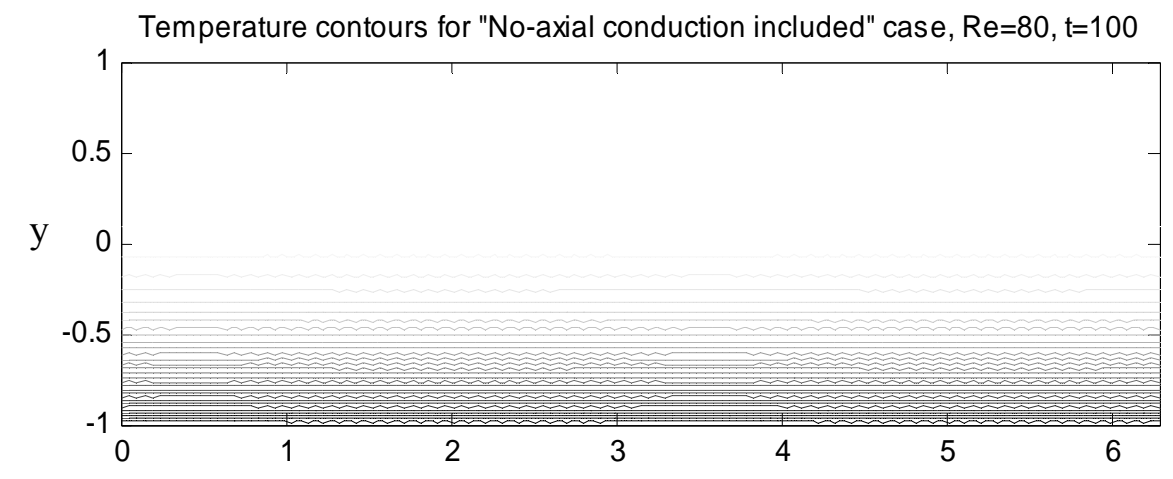

Temperature contours for "No-axial conduction included" case, $\mathrm{Re}=80, \mathrm{t}=120$

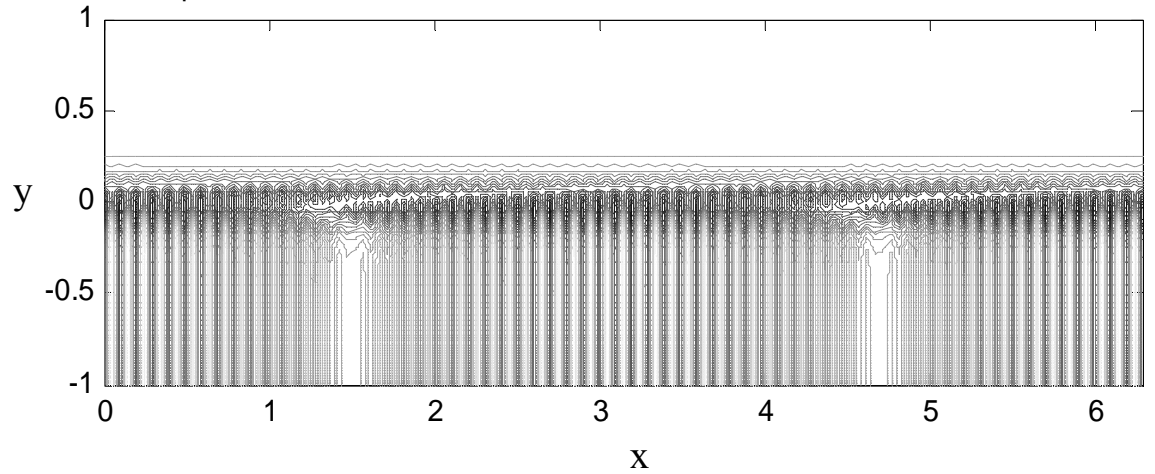

Figure 4.14. For $\mathrm{Re}=80$ no-axial conduction case, temperature contours at $\mathrm{t}^{*}=100,120$. 

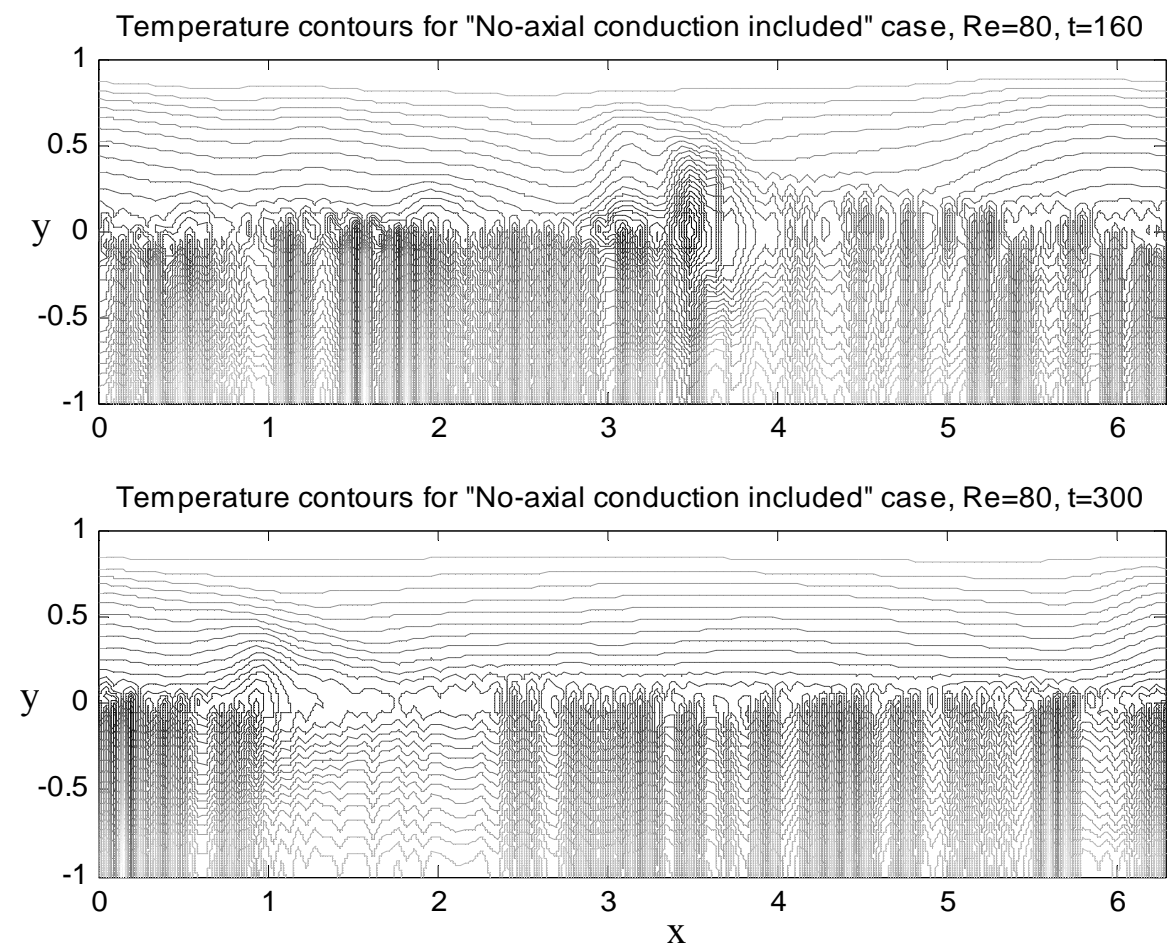

Figure 4.15. For $\mathrm{Re}=80$ no-axial conduction case, temperature contours at

$$
\mathrm{t}^{*}=160 \text { and } 300 \text {. }
$$

\subsubsection{Comparison of the results with literature}

The best way to compare the results of the present work with the literature is to observe the variation of the results in terms of known non-dimensional terms, namely $\mathrm{Nu}, \mathrm{Br}$ and $\mathrm{Re}$. There is very limited data in the literature for us to compare the results of this study. The present work is for parallel plate microchannel and includes natural convection for a completely fully developed flow. Most of the experimental results contain entrance effects, like the one we compare our results with which can easily be a potential cause of the deviation of temperature field from the pure numerical results when entrance and fully developed regions are considered together. Moreover, another cause for the deviation of numerical studies from experimental works can be the impossibility of introducing surface roughness to simulated geometries that seems to be the 
main obstacle in front of having quantitative comparisons. In microchannels, relative roughness becomes very high due to the small hydraulic diameter.

At this point, the present study tries to show the pure effect of the small values of Brinkman number on the microchannel flow. The selection of very low Brinkman numbers reduces the importance of viscous dissipation, thus temperature variations become very small. The $\mathrm{Br}$ values used for the present study are lower when compared with the works in the literature [20]. The lower values affect the single-phase convective heat transfer at the temperature gradient level, which is identified here as the secondary effect of $\mathrm{Br}$. The results obtained in the selected range qualitatively matches with the experimental results [10].

Even for these lower values, it is seen in the work of Tso and Mahulikar [10] that the unusual behavior of $\mathrm{Nu}$ in the laminar regime through the flow of liquids in microchannels could be explained by correlating $\mathrm{Nu}$ with a Brinkman number besides Re and Pr such as:

$$
N u=A \cdot \operatorname{Re}^{0.62} \operatorname{Pr}^{1 / 3} B r^{d}
$$

where A and d are constants.

However, for the present work, the power of Re should have a different value due to different boundary conditions and different cross-section of the channel. The origin of Eqn. 4.11 is the work of Peng and Peterson [1]. For the value of the power of $\mathrm{Br}$, the authors purposed that additional data is needed for a universal correlation

The cases and corresponding non-dimensional numbers are summarized in Table 4.2. The values for Nusselt are within the ranges for $\mathrm{Nu}$ given in the literature. Original values for the selected case presented in Table 4.2 can be seen in the 
work of Peng and Wang [3]. They investigated the phenomena for a broader range of Re.

Table 4.2 Numerical experimentation cases with corresponding non-dimensional numbers

\begin{tabular}{|c|c|c|c|c|c|c|}
\hline & $\mathrm{Br}$ & $\mathbf{R e}$ & Nu & Pr & $\mathrm{Nu} / \mathbf{P r}^{1 / 3}$ & $\mathrm{Nu} /\left(\operatorname{Re}^{0,62} * \operatorname{Pr}^{1 / 3}\right)$ \\
\hline Case 1 & \multicolumn{6}{|c|}{$U_{m}=0.25 \mathrm{~m} \mathrm{~s}^{-1} \mathrm{~L}=3.1 * 10^{-4} \mathrm{~m}$} \\
\hline 1 & $2.8333 \times 10^{-5}$ & 80 & 1.85271 & 6.736 & 0.981 & 0.0648 \\
\hline 2 & $1.8109 \times 10^{-5}$ & 87 & 1.8195 & 6.129 & 0.994 & 0.0624 \\
\hline 3 & $7.5320 \times 10^{-6}$ & 97 & 1.80622 & 5.415 & 1.029 & 0.0603 \\
\hline 4 & $4.5590 \times 10^{-6}$ & 107 & 1.79958 & 4.851 & 1.063 & 0.0587 \\
\hline Case 2 & \multicolumn{6}{|c|}{$U_{m}=0.29 \mathrm{~m} \mathrm{~s}^{-1} \mathrm{~L}=3.1 * 10^{-4} \mathrm{~m}$} \\
\hline 5 & $4.4115 \times 10^{-5}$ & 93 & 1.99216 & 6.719 & 1.056 & 0.0635 \\
\hline 6 & $3.5956 \times 10^{-5}$ & 100 & 1.9656 & 6.193 & 1.07 & 0.0616 \\
\hline 7 & $2.4100 \times 10^{-5}$ & 116 & 1.94567 & 5.234 & 1.121 & 0.0588 \\
\hline 8 & $1.4541 \times 10^{-5}$ & 124 & 1.93903 & 4.856 & 1.145 & 0.0577 \\
\hline 9 & $1.2091 \times 10^{-5}$ & 136 & 1.92575 & 4.373 & 1.178 & 0.056 \\
\hline Case 3 & \multicolumn{6}{|c|}{$U_{m}=0.76 \mathrm{~m} \mathrm{~s}^{-1} L=3.1 * 10^{-4} \mathrm{~m}$} \\
\hline 10 & $3.9100 \times 10^{-4}$ & 250 & 3.22730 & 6.531 & 1.727 & 0.0563 \\
\hline 11 & $2.0133 \times 10^{-4}$ & 273 & 3.18745 & 5.913 & 1.763 & 0.0544 \\
\hline 12 & $1.4853 \times 10^{-4}$ & 300 & 3.16753 & 5.312 & 1.815 & 0.0529 \\
\hline
\end{tabular}

The first step to be followed is to investigate whether the same observation can be made with the other researchers: If the correlation is of the form of Eqn. 4.11, the graph of $\mathrm{Nu} /\left(\mathrm{Re}^{0.62} \operatorname{Pr}^{1 / 3}\right)$ vs. Br should definitely show a linear form on a log-log scale.

We observed that the correlation is of the form of Eqn. 4.11 and the plot of $\left[N u /\left(\operatorname{Re}^{0.62} \operatorname{Pr}^{1 / 3}\right)\right]$ vs. Br shows a linear form on a log scale. The plots for the three cases are shown in Fig. 4.16. The numerical results for the individual cases show the same behavior as the experimental data shown in [10] and follow a linear relation and are positively sloped, justifying the positive exponent of $\mathrm{Br}$ for the case of fluid heated being considered. From the graphs the data for the 
separate cases appear to have a better linear trend than if all the individual points combined. An important conclusion that can be drawn here is; the trend is a straight line for each individual data set which supports the correlation of the laminar forced flow convection with $\mathrm{Br}$, in addition to $\mathrm{Re}$ and $\mathrm{Pr}$.

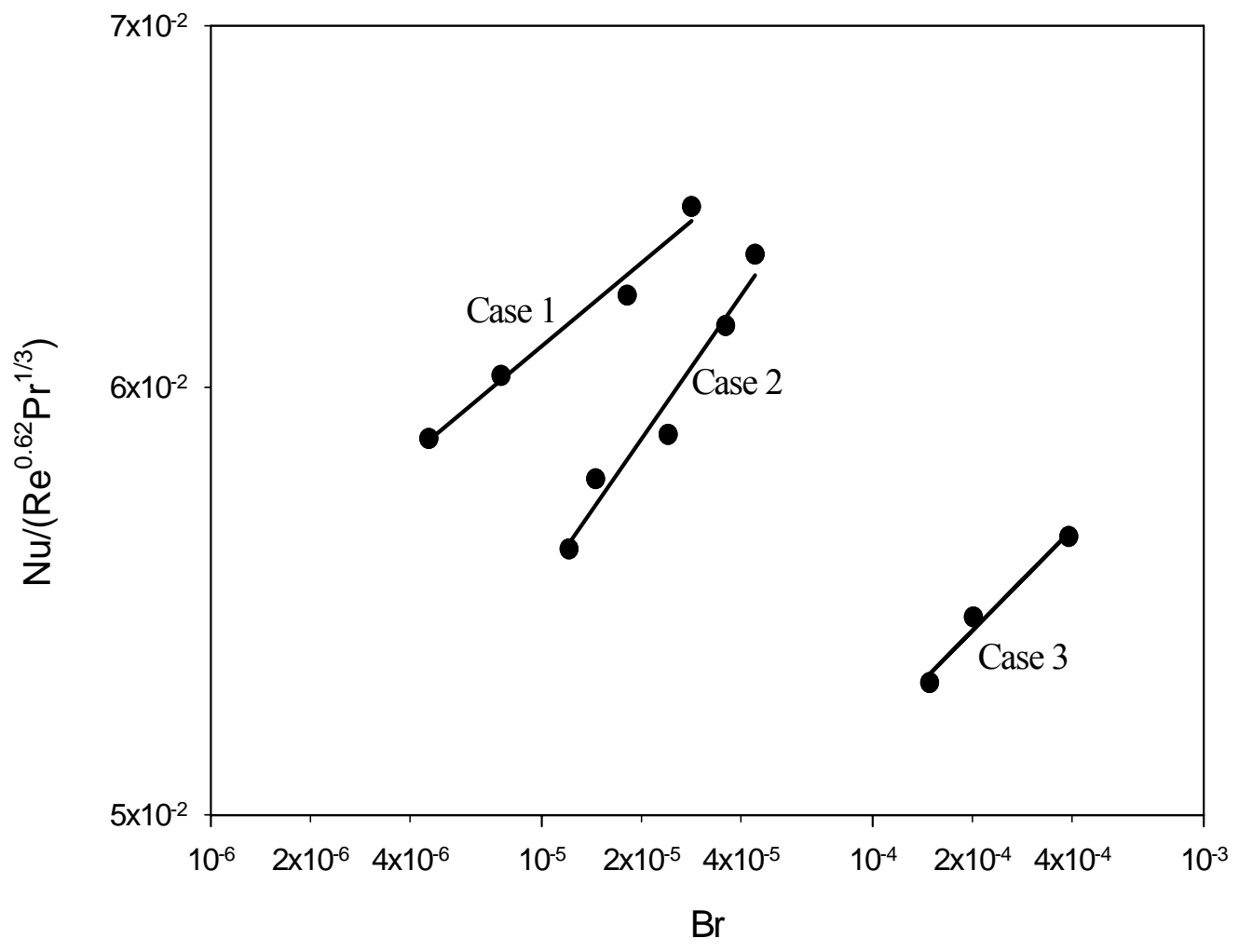

Figure 4.16 Plot of $N u /\left(\operatorname{Re}^{0.62} \operatorname{Pr}^{1 / 3}\right)$ vs. $\mathrm{Br}$ 


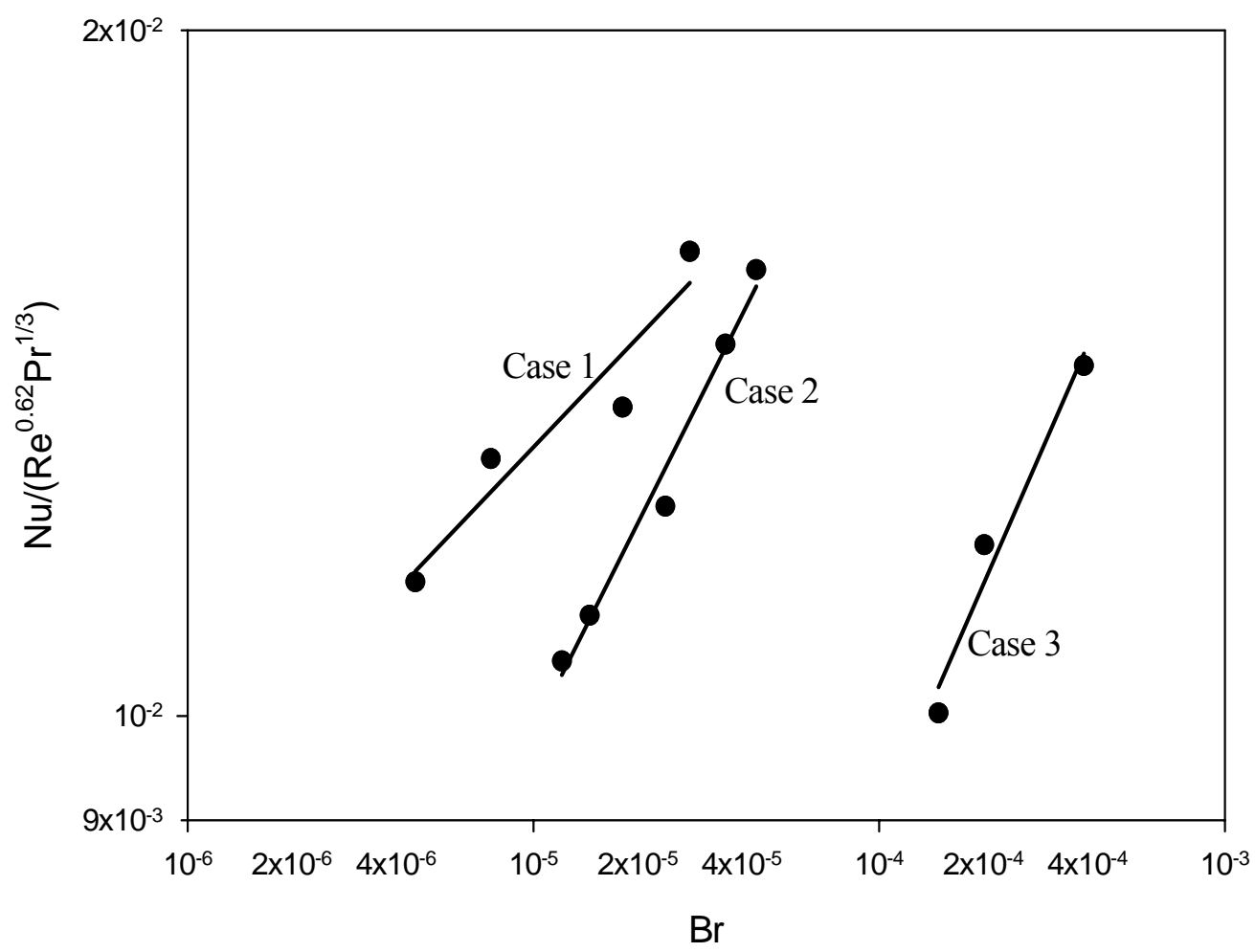

Figure 4.17 Plot of $N u /\left(\operatorname{Re}^{0.62} \operatorname{Pr}^{1 / 3}\right)$ vs. Br, Tso and Mahulikar [10]

Comparing the numerical results in detail with [10], we observe that Tso and Mahulikar observe the same trend. As it can be seen in Figure 4.17 that experimental data points for individual cases follow a linear relation, just as the case for numerical results, and again the best fits are positively sloped. Therefore, our numerical results match with the experimental data, if Figures. 4.16 and 4.17 are compared and the positive exponent of $\mathrm{Br}$ is proved to be valid for both experimental and numerical studies that support Eqn. 4.11. 


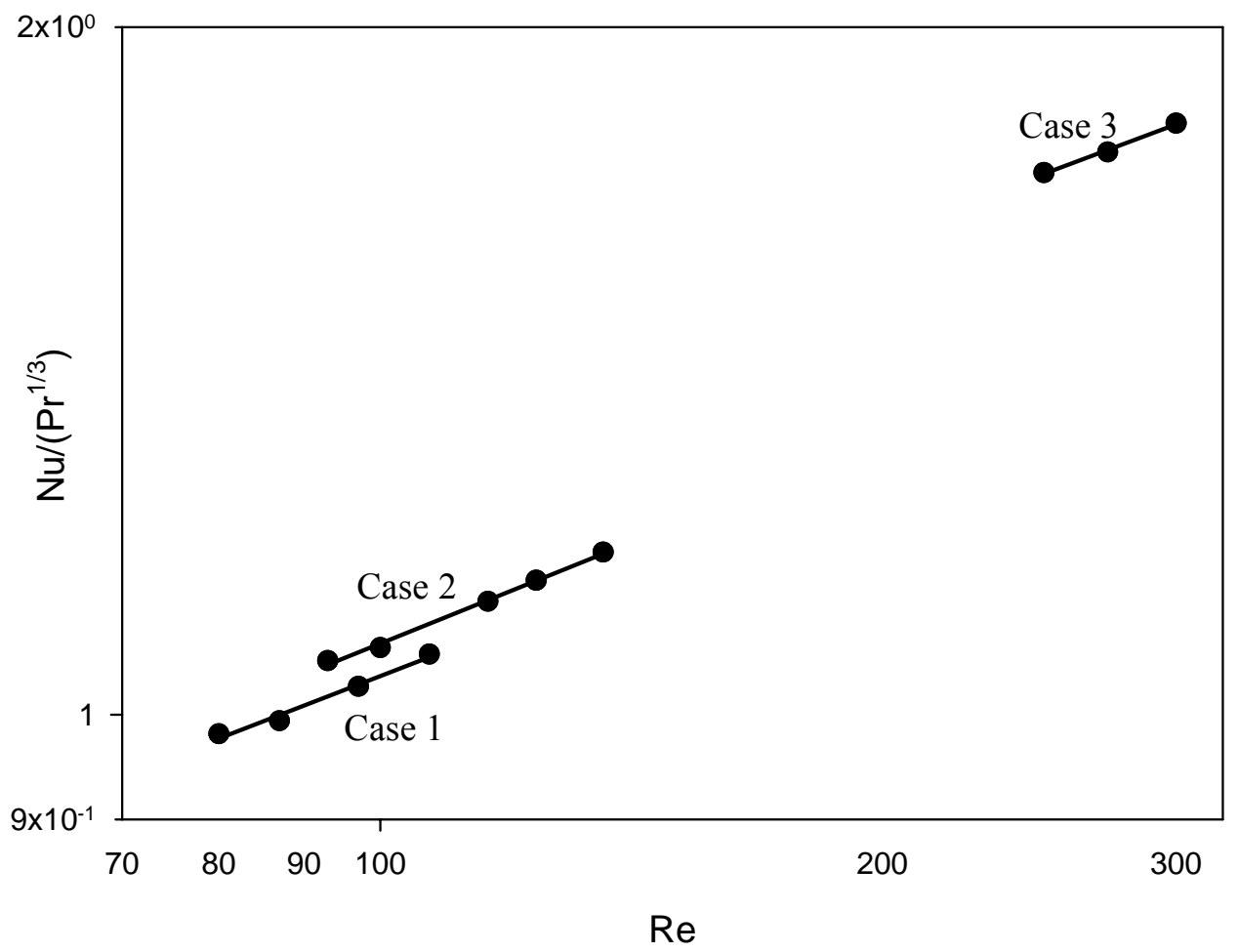

Figure 4.18 Plot of $N u /\left(\operatorname{Pr}^{1 / 3}\right)$ vs. Re

Figure 4.18 shows variation of $N u /\left(\operatorname{Pr}^{1 / 3}\right)$ with Re. There is an increase in the value of $\mathrm{Nu} /\left(\operatorname{Pr}^{1 / 3}\right)$ with an increase in Re. This trend is seen for the three individual cases. The results qualitatively agree with Sieder-Tate correlation used for conventionally-sized channels with viscosity variation and all individual cases give positive slopes, which is an expected result. And the slope of the individual cases ranges between $0.29-0.45$ where 0.33 is expected, which in this case is well predicted by the numerical solution. 


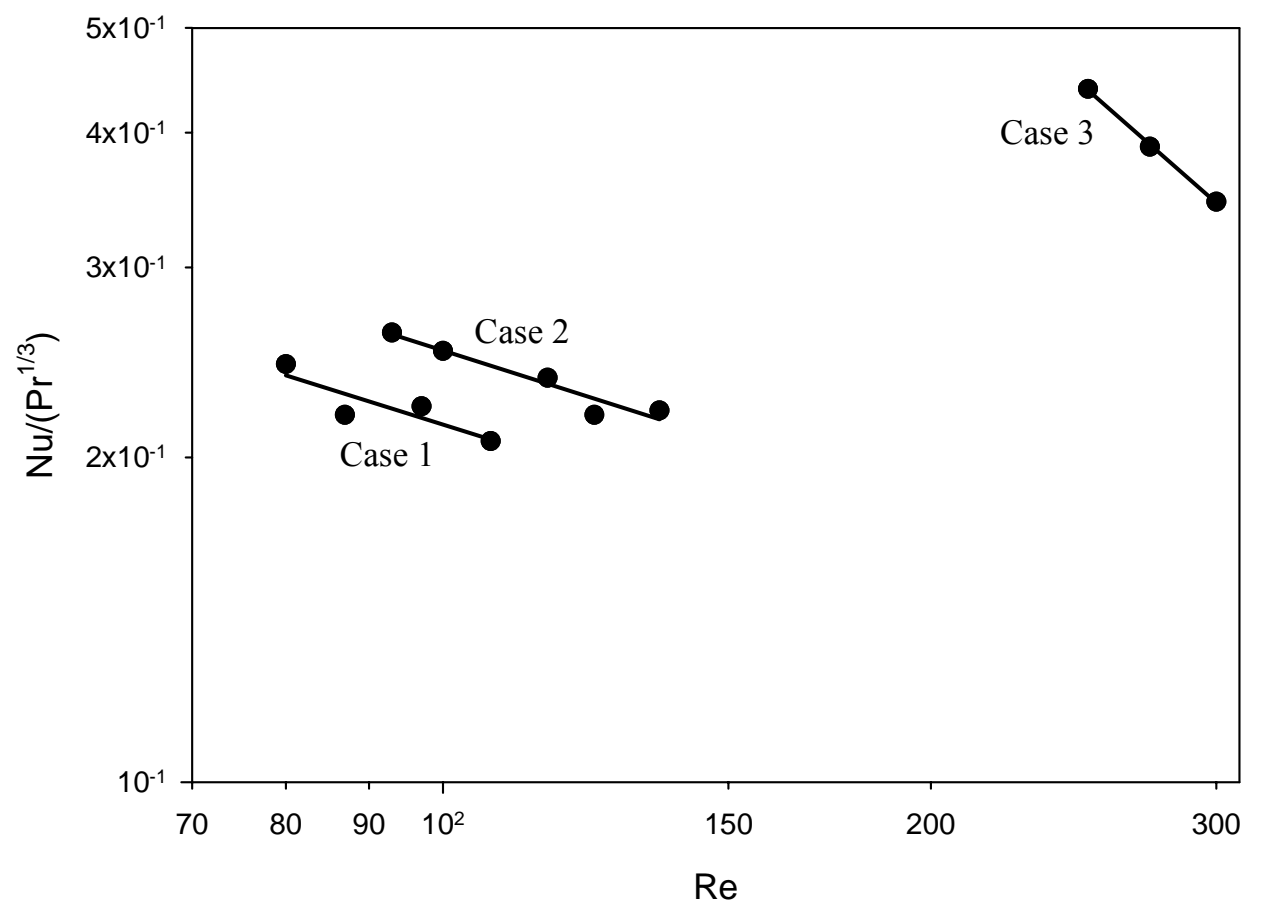

Figure 4.19 Plot of $N u /\left(\operatorname{Pr}^{1 / 3}\right)$ vs. Re, Tso and Mahulikar [10]

From the results of [10], Tso and Mahulikar observed that $N u /\left(\operatorname{Pr}^{1 / 3}\right)$ decreased except for two points. Their result is not parallel with the Sieder-Tate equation. This behavior is unexpected when conventionally sized channels are considered. According to Eqn. 4.11, the slope is expected to be positive. Our results predict qualitatively this number where slight difference is normal because of different numerical experimental set-up between literature and the present work. However, it is observed clearly from Figure 4.19 is that, even though the best fit for all the points combined gives a positive slope, individual cases give unexpected negative values. 


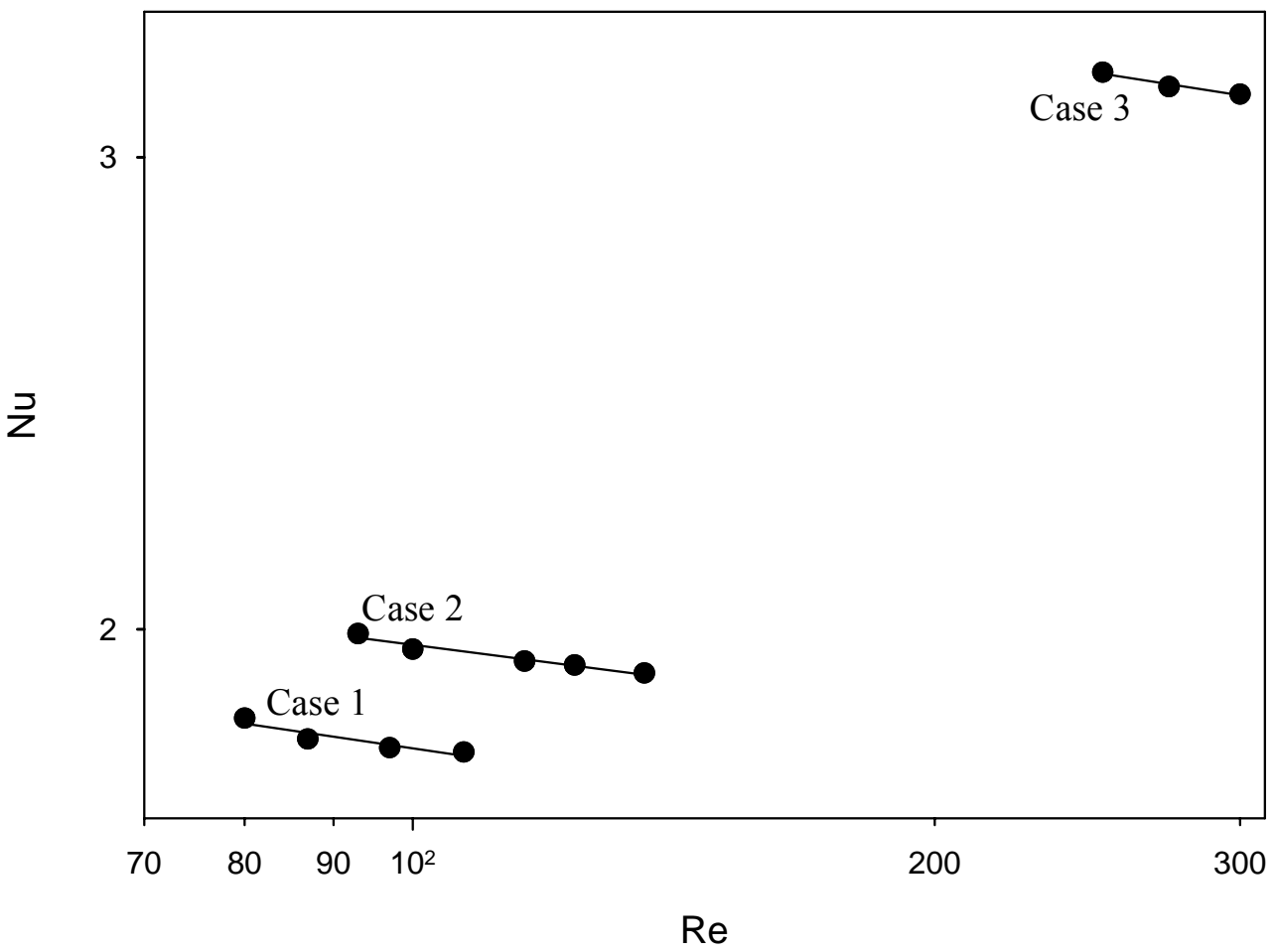

Figure 4.20 Plot of Nu vs. Re

Figure 4.20 shows variation of $\mathrm{Nu}$ with $\mathrm{Re}$. It is seen that Nusselt number is decreasing with increasing Reynolds number for all three cases, which is an unexpected result when conventional channels are considered, since for conventional channels, the average $\mathrm{Nu}$ should increase with increase in average Re over the length, due to $\mathrm{Nu}$ increase in the thermal entry length.

It is also shown in [10] that the unusual behavior of $\mathrm{Nu}$ decreasing with increasing $\mathrm{Re}$ for the case of fluid heated in microchannels is not only a characteristic of a thermally-developing flow in microchannels. 
When compared with Figure 4.21 it is concluded that Tso and Mahulikar observed the same behavior of $\mathrm{Nu}$ decreasing with increasing $\mathrm{Re}$ in the their experimental study

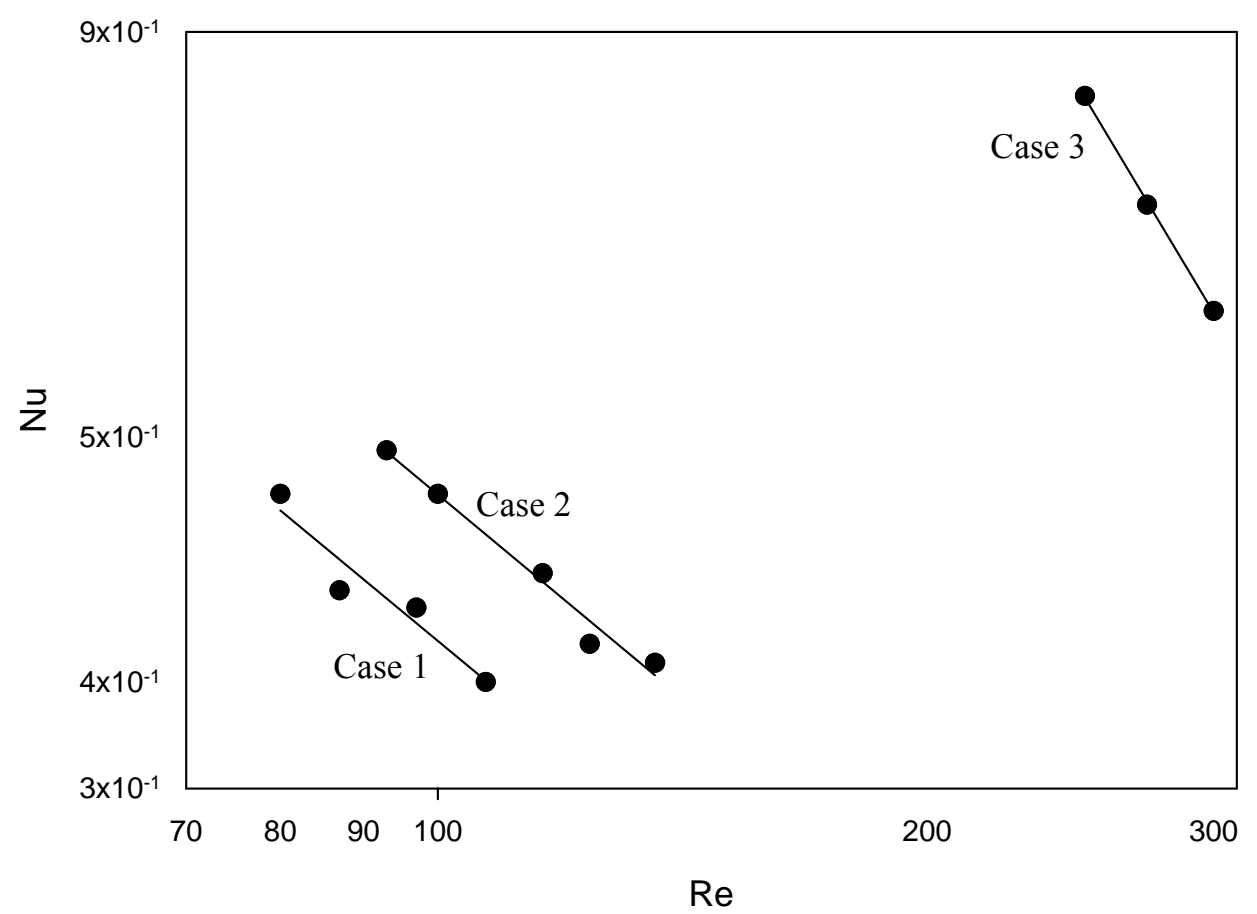

Figure 4.21 Plot of Nu vs. Re, Tso and Mahulikar [10]

Since $\mathrm{Br}$ shows the relative importance of viscous dissipation, it plays different roles for the cases of fluid heated and cooled. For the fluid being heated, the exponent $d$ of $B r$ in the Eqn. 4.11 should be positive, because the $\mu V^{2}$ term also tends to increase the coolant temperature and so the Nusselt number. At a constant velocity, Re increases due to reduction in the coolant viscosity because of the temperature rise of the fluid simulated by taking different $\mathrm{Re}$ into consideration for the same case. So, the $\mu V^{2}$ term decreases and $\left(\mathrm{Re}^{0.62} \mathrm{Br}^{d}\right)$ decreases. At this point it can be seen that the unusual behavior of 
decreasing of $\mathrm{Nu}$ with increasing $\mathrm{Re}$ in the laminar regime of microchannels is explained.

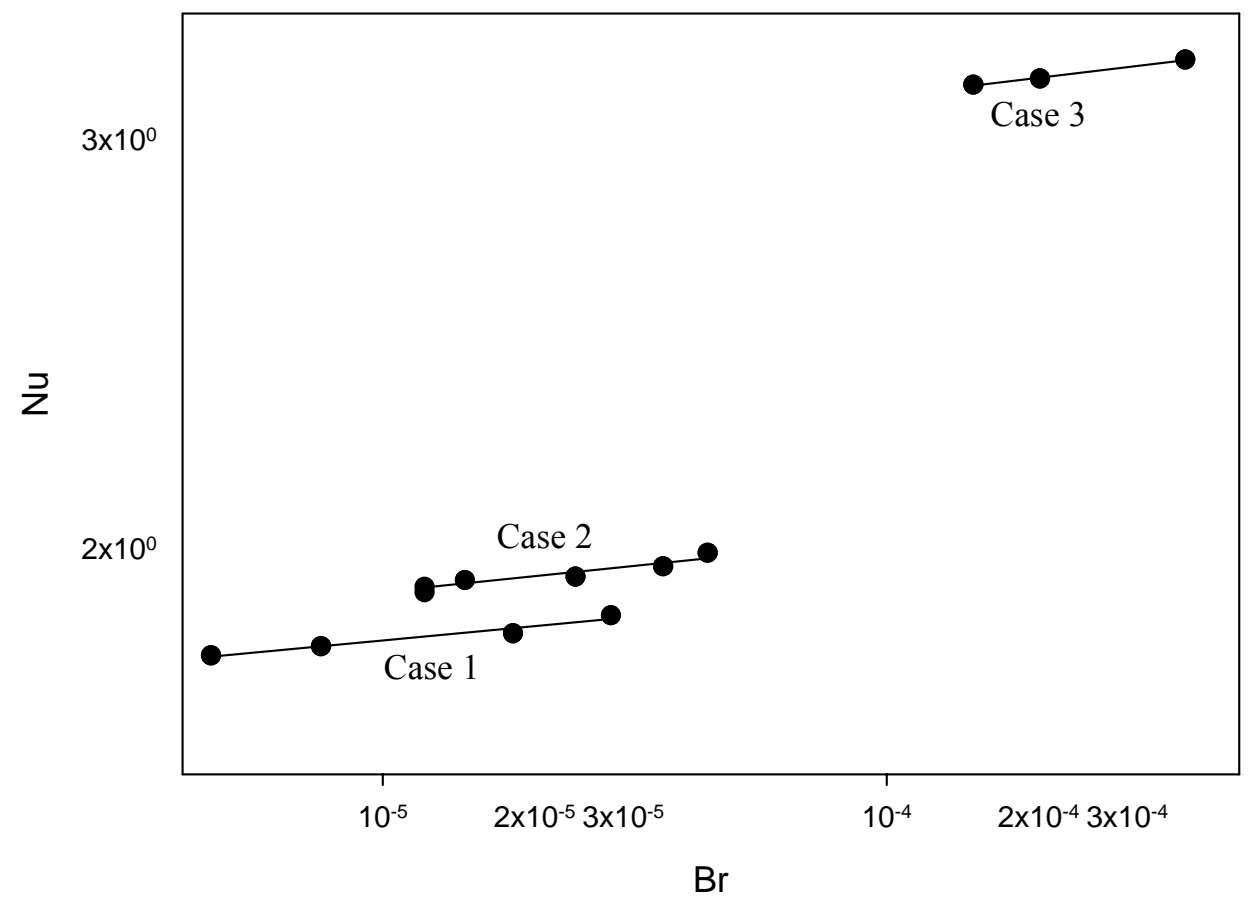

Figure 4.22 Plot of Nu vs. Br 


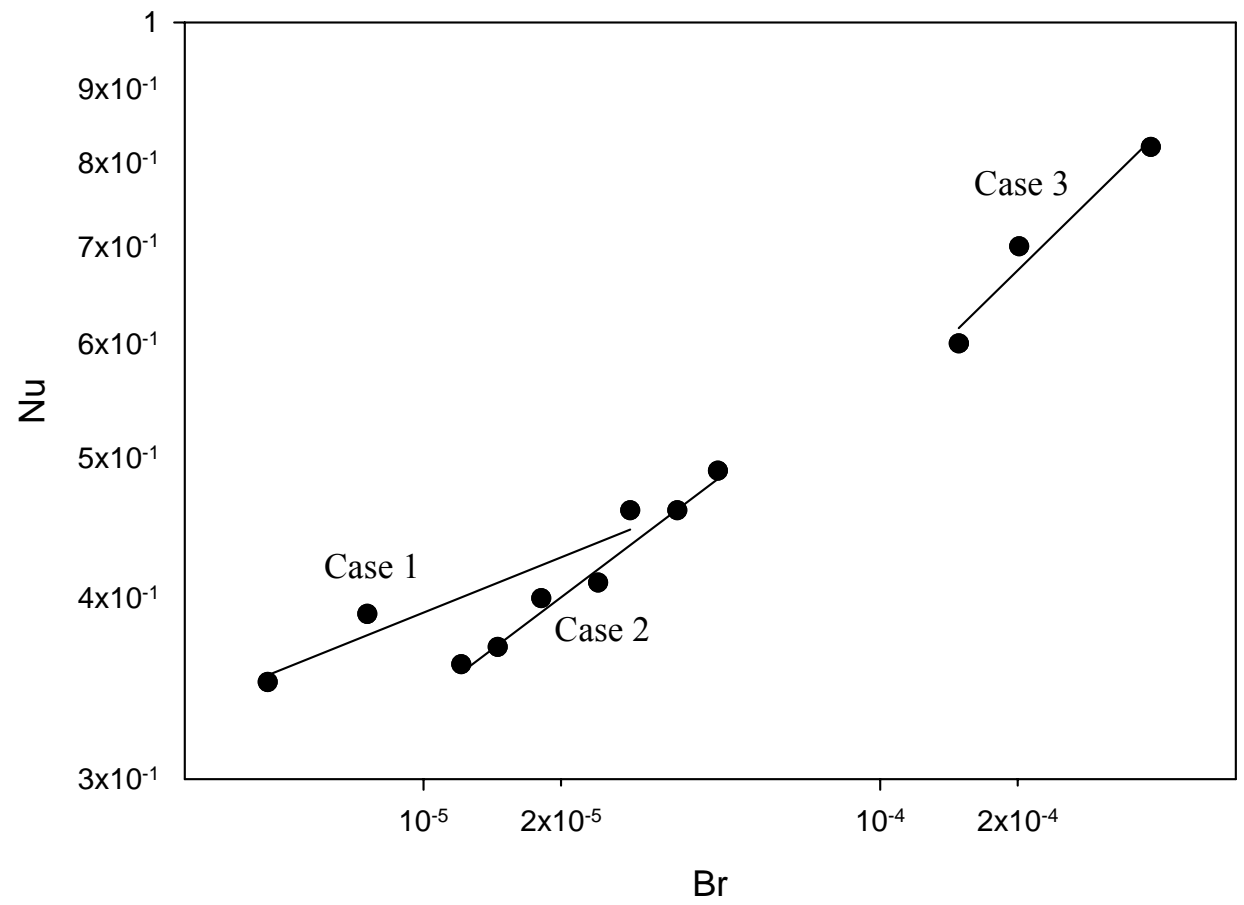

Figure 4.23 Plot of Nu vs. Br, Tso and Mahulikar [10]

Figures 4.22 shows the fact that in the laminar regime, numerical data for $\mathrm{Nu}$ correlates well with the Brinkman number in addition to Re, Pr shown in the Eqn. 4.11, therefore explaining the unusual behavior by having a positive linear trend. The linear trend is seen easier on the log-log scale than on a linear scale shown in Figure 4.24.

This dependence of $\mathrm{Nu}$ by $\mathrm{Br}$ is also observed physically by the authors of [10]. Therefore, we observe same trend in both Figures 4.22 and 4.23. The data points of individual cases show the same positive linear trend just as the results of the present numerical study. 


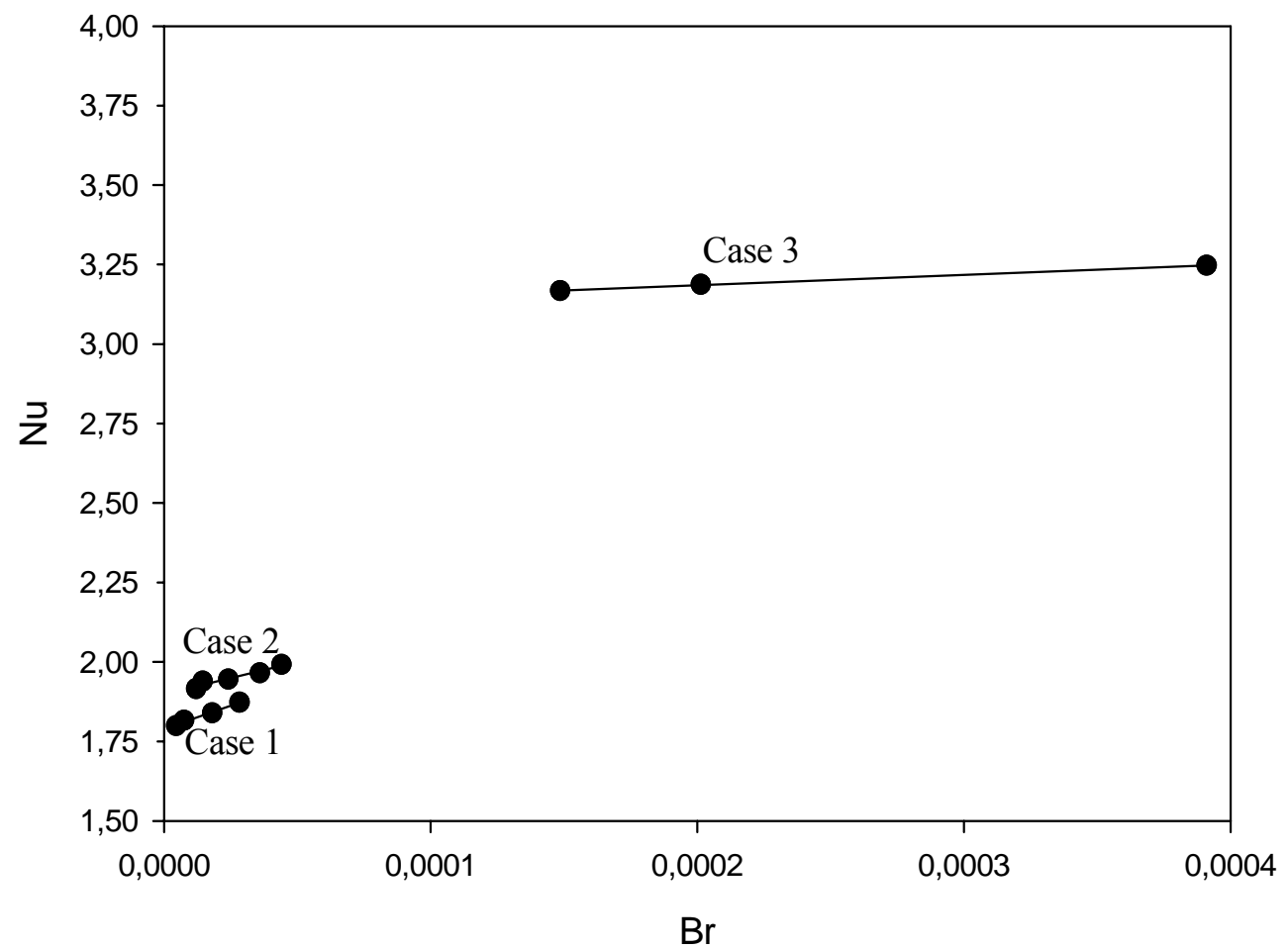

Figure 4.24 Plot of $\mathrm{Nu}$ vs. Br on a linear scale

As it is mentioned in [10], this dependence of $\mathrm{Nu}$ to $\mathrm{Br}$ is the answer to the observation made by Wang and Peng [3]. For $\operatorname{Re}<700$ they investigated this unexpected behavior and reach the conclusion that $\mathrm{Nu}$ appeared to be a function of some other unknown variations besides Re. This unusual behavior is explained in the studies of Tso and Mahulikar $[10,11]$. We also observe a similar behavior which is seen in the experimental study given in [11]. Figures 4.23 and 4.25 show the results in log-log and linear scales, respectively. 


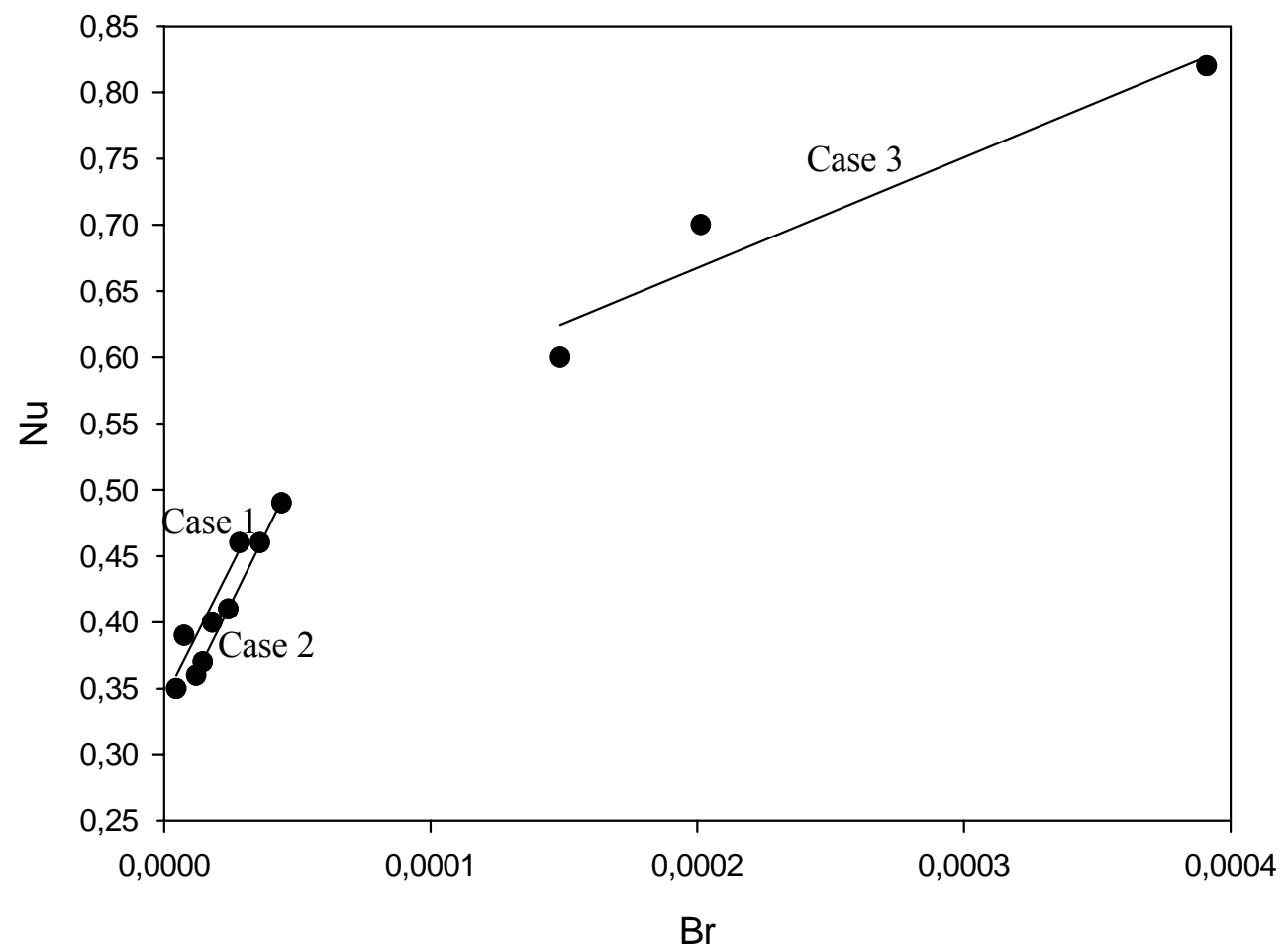

Figure 4.25 Plot of Nu vs. Br on a linear scale, Tso and Mahulikar [10]

The exponents shown in Eqn. 4.11 are expected to be different from the work of Tso and Mahulikar [10] due to two main and important reasons:

1) Boundary conditions: The boundary condition for [10] is constant heat flux but it is constant wall temperature in this work.

2) Geometry of the microchannel: channel with rectangular cross-section for [10] but parallel plate channel for the present work. It is widely accepted by the authors of the previous studies $[3,46]$ that the geometric parameters significantly affect the flow and hence the convective heat transfer. Therefore, observing such a difference in quantity is something expected. 
The general trends and unusual behaviors found in experimental work are well simulated and observed in this work. For liquid flow in microchannels, dissolved gases in the liquid or gases absorbed on the surface may have considerable impact on the flow and heat transfer characteristics. As such gases accumulate in the corners of non-circular channels, the wetted perimeter will be reduced and the fluid velocity will increase as the actual flow cross-section is reduced. The smaller perimeter reduces the friction while the large velocity increases the friction. This may also be a cause for the slight difference between experimental and numerical works which is widely seen in literature [45].

It is seen in the above results that $\mathrm{Nu}$ is well correlated with the $\mathrm{Br}$ even if the value of $\mathrm{Br}$ is very small. At this specific point one important question arises: Are there any differences between the linear trends shown in $\mathrm{Nu}$ vs. Br plot for all orders of magnitude of Brinkman number?

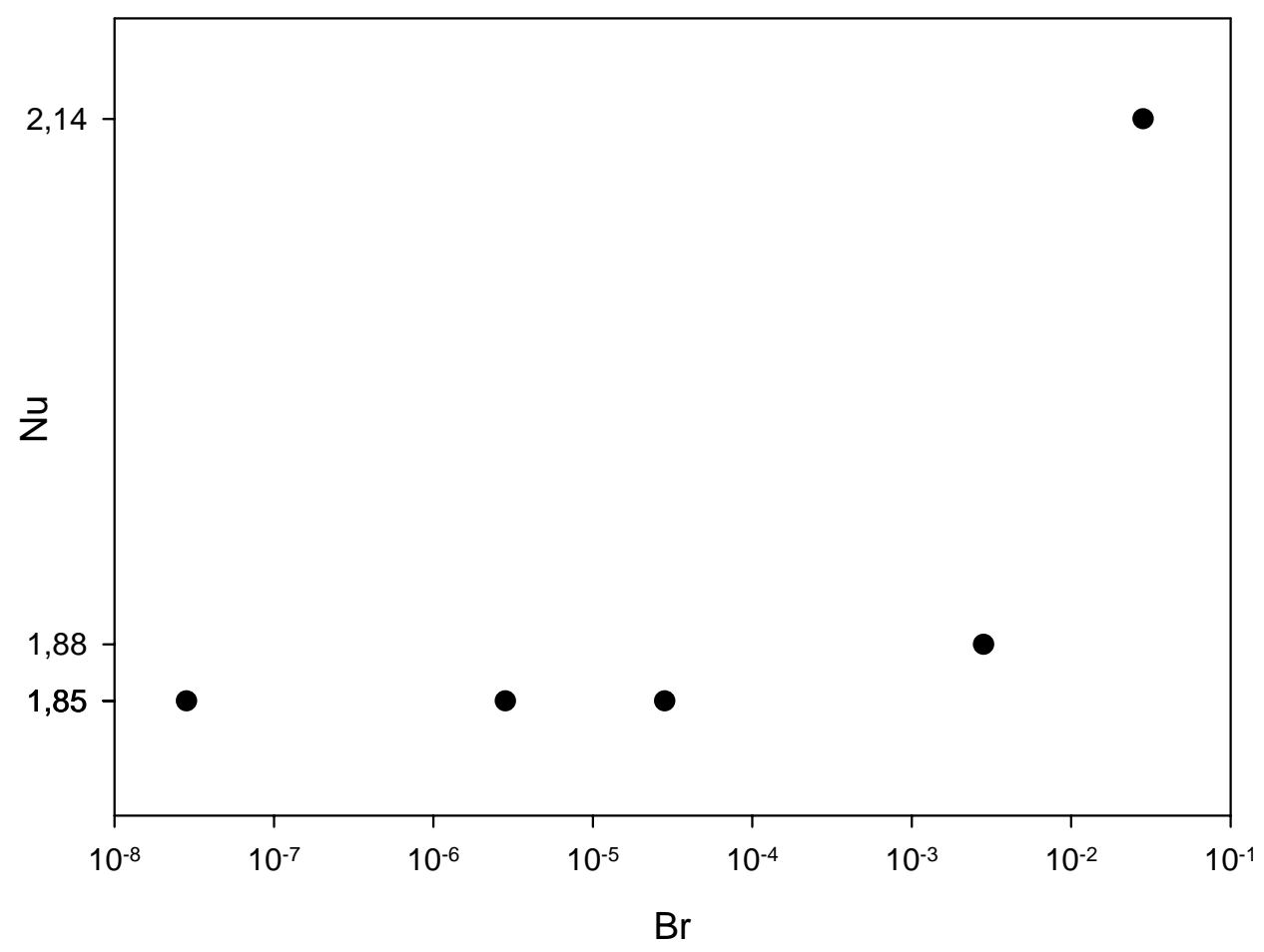

Figure 4.26 Plot of Nu vs. Br for $\mathrm{Re}=80$. 
Figure 4.26 demonstrates the answer of the question above. It is easily seen in the figure that $\mathrm{Nu}$ seems to be constant for low values of $\mathrm{Br}$. After the value of $10^{-3}$, the effect of $\mathrm{Br}$ on $\mathrm{Nu}$ is more obviously seen. The physics behind this phenomenon is the difference between primary and secondary effects of $\mathrm{Br}$. It is accepted in the literature $[10,11]$ that when the $\mathrm{Br}$ is very large, it affects directly due to the effect of viscous dissipation, even for a constant value of $\mathrm{Br}$ along the flow, and this effect is called the primary effect of $\mathrm{Br}$.

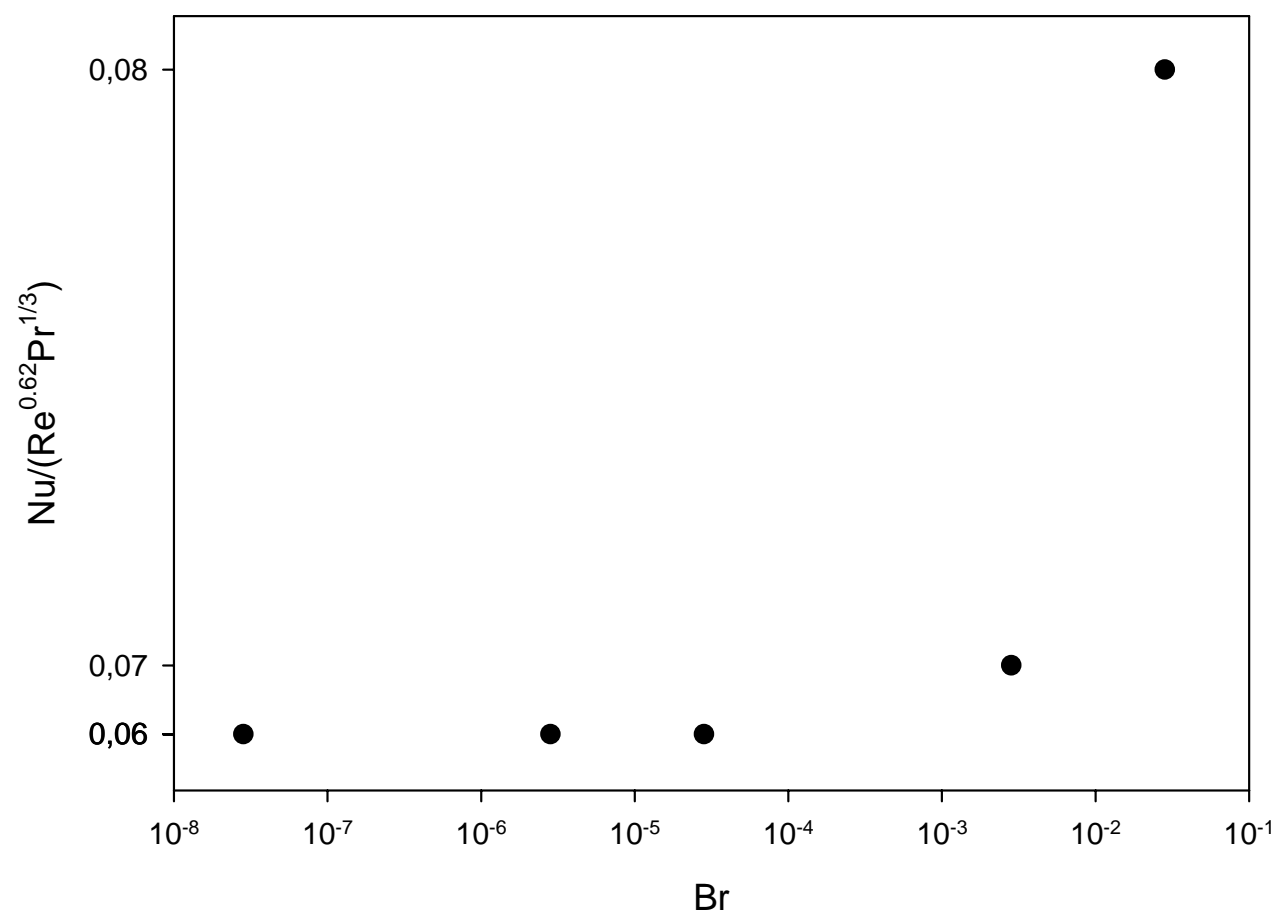

Figure 4.27 Plot of $\mathrm{Nu} /\left(\mathrm{Re}^{0.62} \mathrm{Pr}^{1 / 3}\right)$ vs. $\mathrm{Br}$ for $\mathrm{Re}=80$.

The secondary effect of $\mathrm{Br}$ is hard to observe in the numerical experimentation since it is closely related with the variations in the coolant properties which in return cause the variation in $\mathrm{Br}$ in microchannels. However, it is hard to simulate this phenomenon in numerical experimentation. And this is a difficulty faced by many authors in most of the numerical work for gases in the literature $[19,20]$. 
Figure 4.27 is definitely another clear observation of $\mathrm{Nu}$ correlating with $\mathrm{Br}$ for laminar flow in microchannels. It is seen from the figure that the correlation between $\mathrm{Nu}$ with $\mathrm{Br}$ independent of $\mathrm{Re}$ and $\mathrm{Pr}$ is getting stronger when $\mathrm{Br}$ has higher values. The primary effect of $\mathrm{Br}$ could also be seen from this graph by making an analogy with the literature [10]. 


\section{CHAPTER 5}

\section{NATURAL CONVECTION IN MICROCHANNELS}

The numerical set-up used in the present work is naturally unstable because of its top-heavy arrangement. This is the very first work done on the literature about natural convection in microchannels.

Because of the temperature difference between the top and bottom plates an inverse density gradient occurred due to the fact that the fluid is lighter at the bottom than the top because the channel is heated from below. Because of the top heavy arrangement it is a potentially unstable flow. The fluid tends to get a new place to overcome this natural weakness coming from its arrangement. Nevertheless, its own viscosity again restricts this tendency taken by the fluid. Consequently, it is expected when the temperature gradient exceeds a certain value for the instability to occur, which makes Rayleigh number very important for the case at hand.

In the late $19^{\text {th }}$ century, Rayleigh and Benard studied the thermal instability in fluids. There are two very important outcomes of its work:

- The fluid must achieve a specified critical temperature gradient before the instability can be observed.

- Passing that critical gradient one can observe a stationary cellular character. The cells have structure very similar to a series of vortices.

After Benard's observations, Rayleigh demonstrated a non-dimensional number (Ra) that expresses the sufficient condition for the onset of the instability. The critical $\mathrm{Ra}$ for the onset of convection is accepted to be 1708 . Heat conduction is the major mode of heat transfer below this specific limit. However, above this 
critical number the dominant mode of heat transfer is convection and the flow consist of two dimensional rolls which have nearly square cross-section and counter-rotating. These rectangular cross-section rolls are well known to be Rayleigh-Benard cells. However, it should be mentioned that the flow becomes complicated as the flow exceeds the critical value more than one order of magnitude. What is observed after this high order of magnitude Ra is that the flow becomes $3 \mathrm{D}$ and the rolls should be investigated in $3 \mathrm{D}$, and the characteristic shape is hexagonal in nature. When Ra takes a value higher than the values mentioned, the flow becomes turbulent and oscillatory eventually. The value of this second critical Ra depends strongly on the geometry and Prandtl number. And it is observed in the literature [43] that with increasing $\mathrm{Ra}$, the stability of the cells decreases.

Because of the restricting nature of the microchannels we do not expect $\mathrm{Ra}$ to exceed the critical value, 1708. Additionally, for microchannels it is physically impossible to reach this $2^{\text {nd }}$ critical value, where the flow becomes $3 \mathrm{D}$, because of the small characteristic length.

In the vertical direction the rolls of the Rayleigh-Benard cells augment the net heat transfer. The reason is the difference between pure conduction and convection. When $\mathrm{Ra}$ is less than the critical value, the mode of heat transfer is conduction.

We expect the cells to be 2D for $\mathrm{q}=$ sinx case, which means a periodically heated lower wall, but the solver is also run in $3 \mathrm{D}$ for verification and observation. According to this, it is expected from the natural convection in microchannels not to reach turbulence because of this geometrical restriction.

The geometry of the problem used for the natural convection is the same as used for forced convection cases, Figure 1.1. 
Because natural convection is investigated in this chapter, one can easily guess that there should be no initial velocity, consequently no initial velocity profile.

\subsection{Heat Transfer Analysis}

The basic solution used for obtaining the results for natural convection in microchannels is the same method used in Chapter 4. Three cases 1) Axial conduction included 2) No-axial conduction and 3) Br number inserted into the energy equation are investigated.

\subsubsection{Numerical Formulation}

The selected cases by selecting working fluid as liquid water are: a small value of $\mathrm{Ra}=25.219$ and a large value of $\mathrm{Ra}=2000$. A reversed procedure is followed compared to the forced flow procedure described in Chapter 4, here we start with the knowledge of Ra. Since we have no data about natural convection in microchannels in the literature, we rely on the non-dimensional numbers of conventional channels. Since our channel is numerically simulated in Chapter 4 by using the properties and the characteristic length, we can adapt the same methods for the natural convection. At this specific point we have a problem due to the size restriction of the microchannel. This restriction arises because when we want to use $\mathrm{Ra}=2000$ with such a small characteristic length of a microchannel we should either have an extremely large temperature difference between the plates or we should alter the gravitational acceleration value. The former is physically impossible because of the small channel height. However, we can simulate the latter assuming a centrifugally generated acceleration.

In order to simulate real life conditions of flow in microchannels, we also investigated the flow both in $2 \mathrm{D}$ and $3 \mathrm{D}$ for $\mathrm{Ra}=25.219$. This number is chosen because of the reasons arising from the conditions of Chapter 4. It simulates a real life condition without a centrifugal effect and a reasonable temperature difference between two walls of the channel. Although we know that for 
conventional channels such kind of small value of Ra means heat transfer by conduction, the case might be different for microchannels. With the help of artificial temperature field disturbance created by the program we are able to simulate cell formations. The disturbance simulates the property variations that occur naturally in real life conditions. It is inserted into the computer code by altering the non-dimensional temperature field by a certain value only at some specific collocation points.

For the numerical experimentation, first we decide which non-dimensional numbers we should use within the limits of the conventional channel critical values. Therefore, some of the numbers should have been fixed to obtain the others. Hence, the three numbers fixed are: $\mathrm{Ra}, \mathrm{Br}$ and characteristic length $\mathrm{L}$. At this point we followed exactly the same procedure but in reverse with Tso and Mahulikar [10] to obtain the other necessary numbers. All the necessary property values of water are assumed to be the same as the properties of case 1 with $R e=80$ case in Table 4.1. After these critical points, our gravitational acceleration is adjusted according to these conditions at hand. The value for using $\mathrm{Ra}=2000$ is $777.184 \mathrm{~m}^{2} / \mathrm{s}$, which is approximately $77.7 \mathrm{~g}$. This value of $\mathrm{G}$ is acceptable since one can see machines with $1000 \mathrm{~g}$ in commercial markets. And it is generally accepted that it is the term gravitational acceleration times density that is responsible for phenomena of convection.

For natural convection again three different concepts with different cases are inspected. The reason of choosing these data is the lack of experimental data for our parallel plate microchannel numerical experimentation case for natural convection in microchannels. 


\subsection{Numerical Verifications}

\subsubsection{Grid Independence}

Like the study done in Chapter 4, the only method to determine grid independence is to setup a model with a finer mesh (a larger number of collocation points) and analyze it to see if there are major differences in velocity and temperature fields. First the case with 64 and 32 points in $y$ and $x$ directions, respectively, is selected $(64 \times 32$ grid). To show the grid independence for the same case, a run with $128 \times 64$ grid was tried and no difference is observed with the coarser grid results. Same results are obtained for the temperature fields. The results for the streamline contours are shown in Figure 5.1. Consequently, it is concluded that $128 \times 64$ or $64 \times 32$ are both sufficient meshes for the work at hand and $64 \times 32$ is chosen as the numerical experimental mesh.
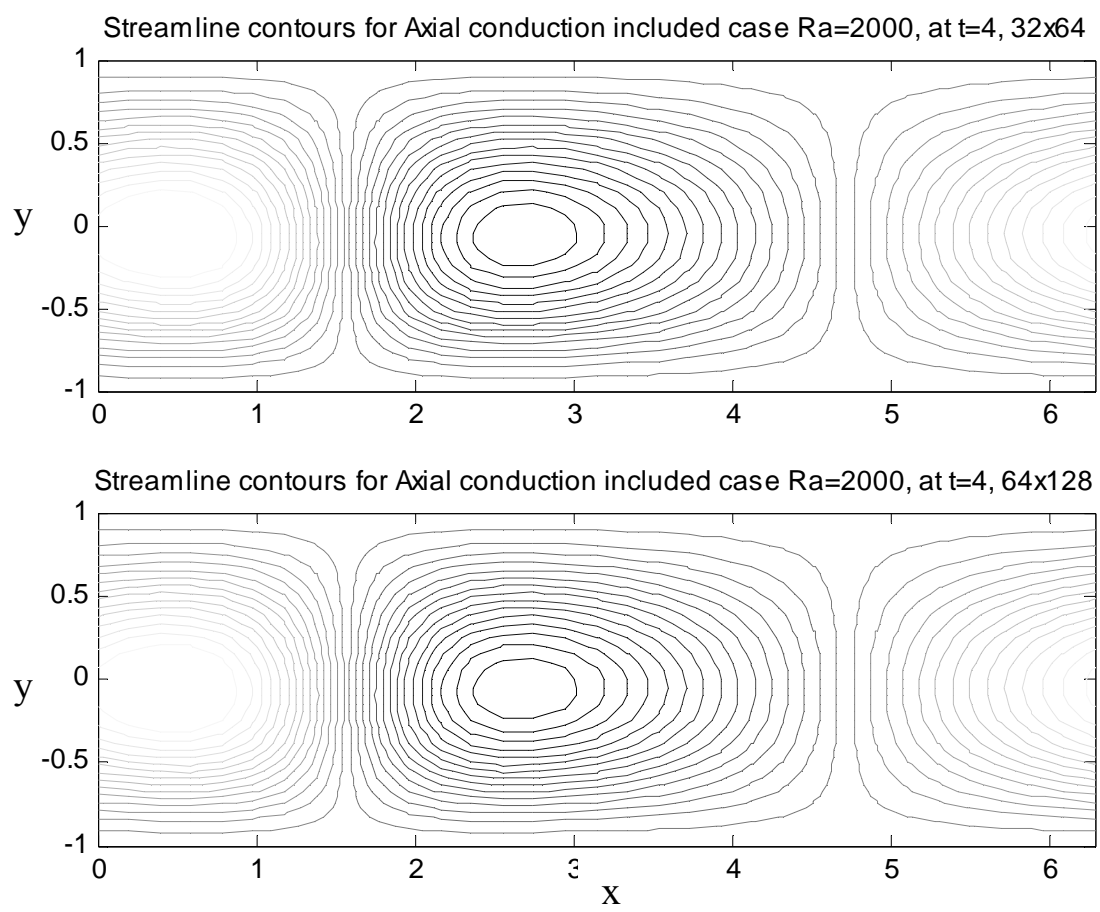

Figure 5.1. Streamline contours for $32 \times 64$ mesh and $64 \times 128$ mesh 


\subsubsection{Diffusion Time Scale Determination}

After determining the experimentation mesh the next step is finding the time scale, $d t$. We began with $\mathrm{dt}=0.001$ and $64 \times 32$ combination. After obtaining the results, the code is rerun for the same conditions and mesh except that this time the time scale is inserted as $\mathrm{dt}=0.0001$. It is seen in Figure 5.2 that the diffusion time scale can be taken as $\mathrm{dt}=0.001$ during the numerical experimentation for $2 \mathrm{D}$ simulations since the results for both of these two configurations are the same.
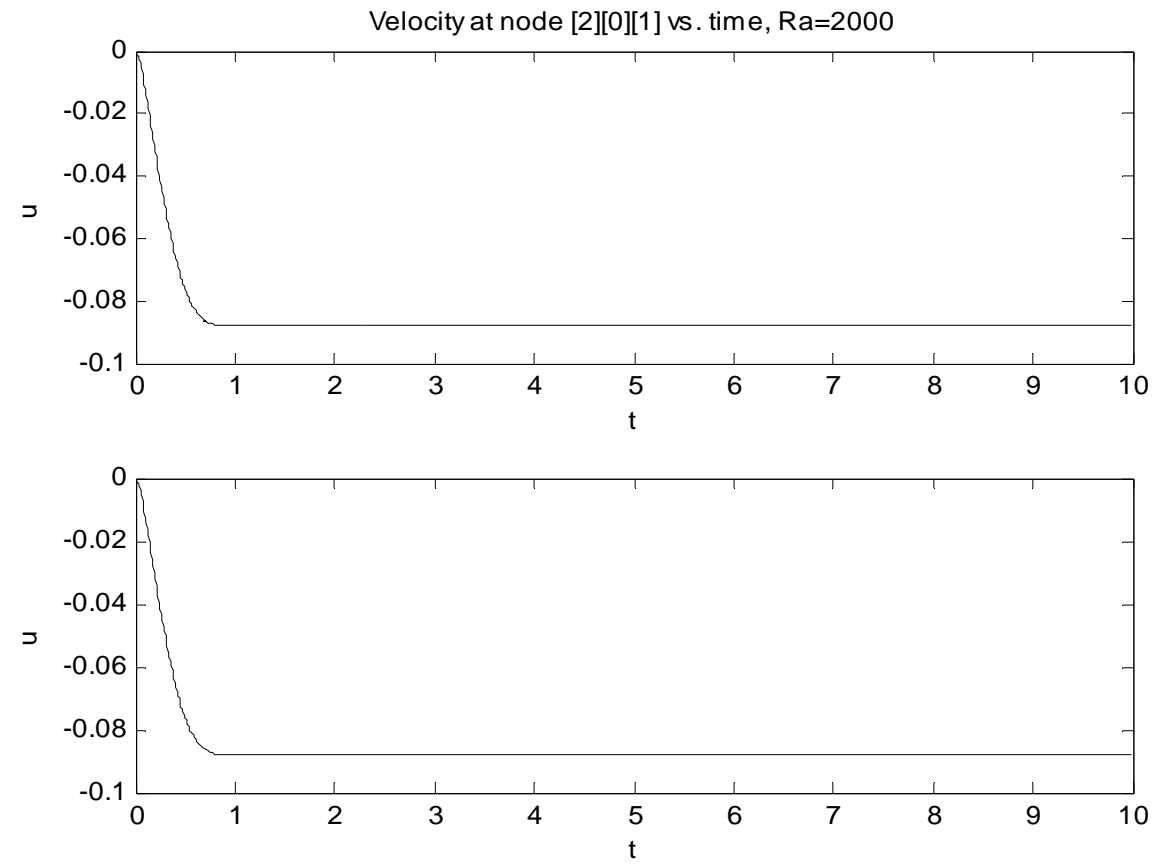

Figure 5.2. Time change of velocity at node [2] [0] [1] for $10^{-3}$ and $10^{-4}$

\subsection{Results and Discussion for 2D}

\subsubsection{Effect of Br for Natural Convection}

For the three different cases investigated. From Figures 5.3-5.4, it is observed from the streamline contour plots that there is not a significant difference between 
the case with axial conduction inclusion and Brinkman number inserted into the energy equation because of the nature of the Brinkman number definition. For the reason of the importance of gravitational acceleration times density for convection in Rayleigh-Benard cells, it is widely accepted that the heat release due to viscous dissipation is negligible for conventional channels which supports our conclusion for microchannels. Therefore, we conclude that although $\mathrm{Br}$ is very important for forced convection we do not have this strong effect seen in natural convection. Moreover, the case of "no-axial conduction" shows the same behavior with the other two cases.
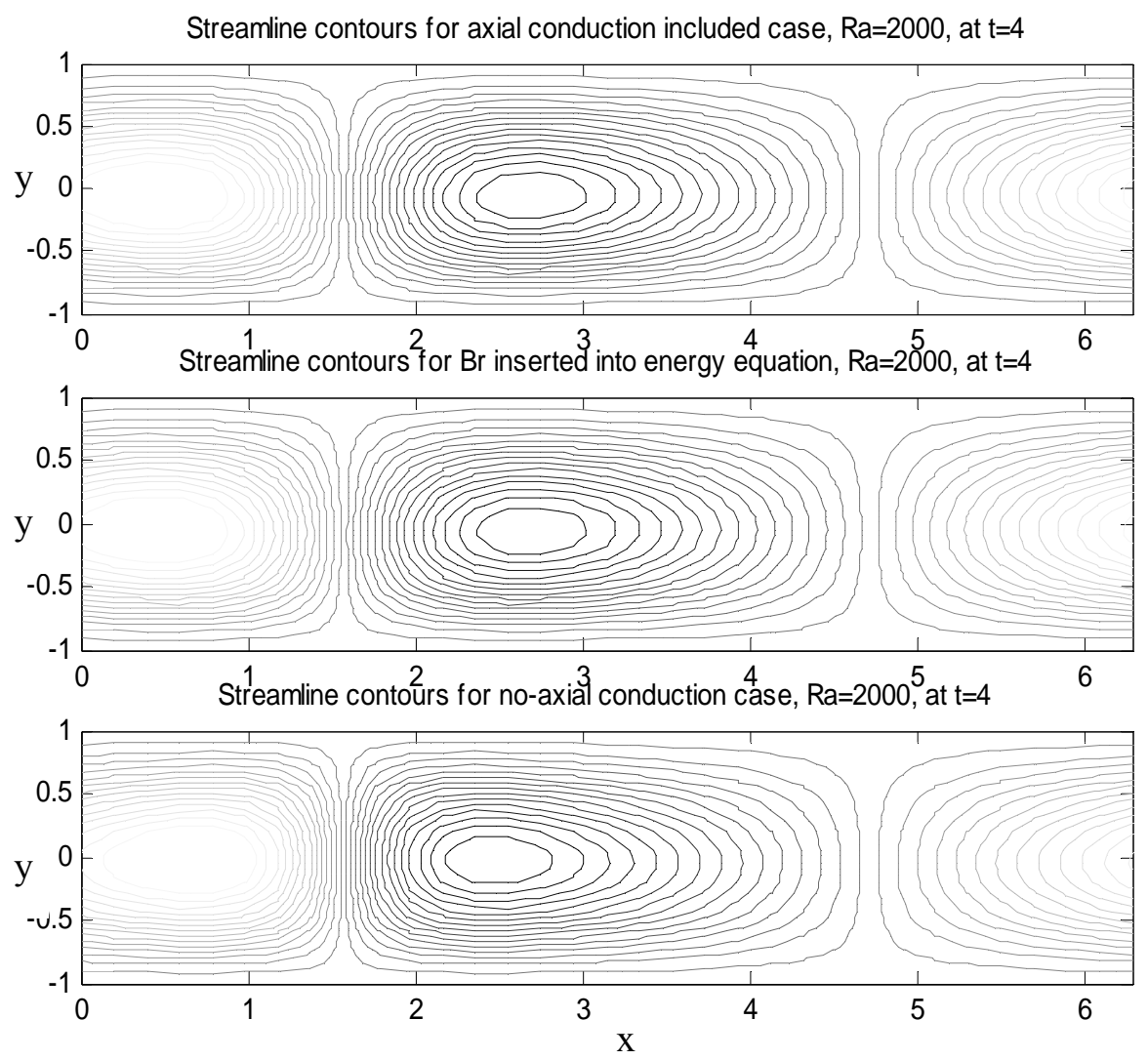

Figure 5.3. Streamline contours for axial conduction included, $\mathrm{Br}$ and No-axial conduction included case 

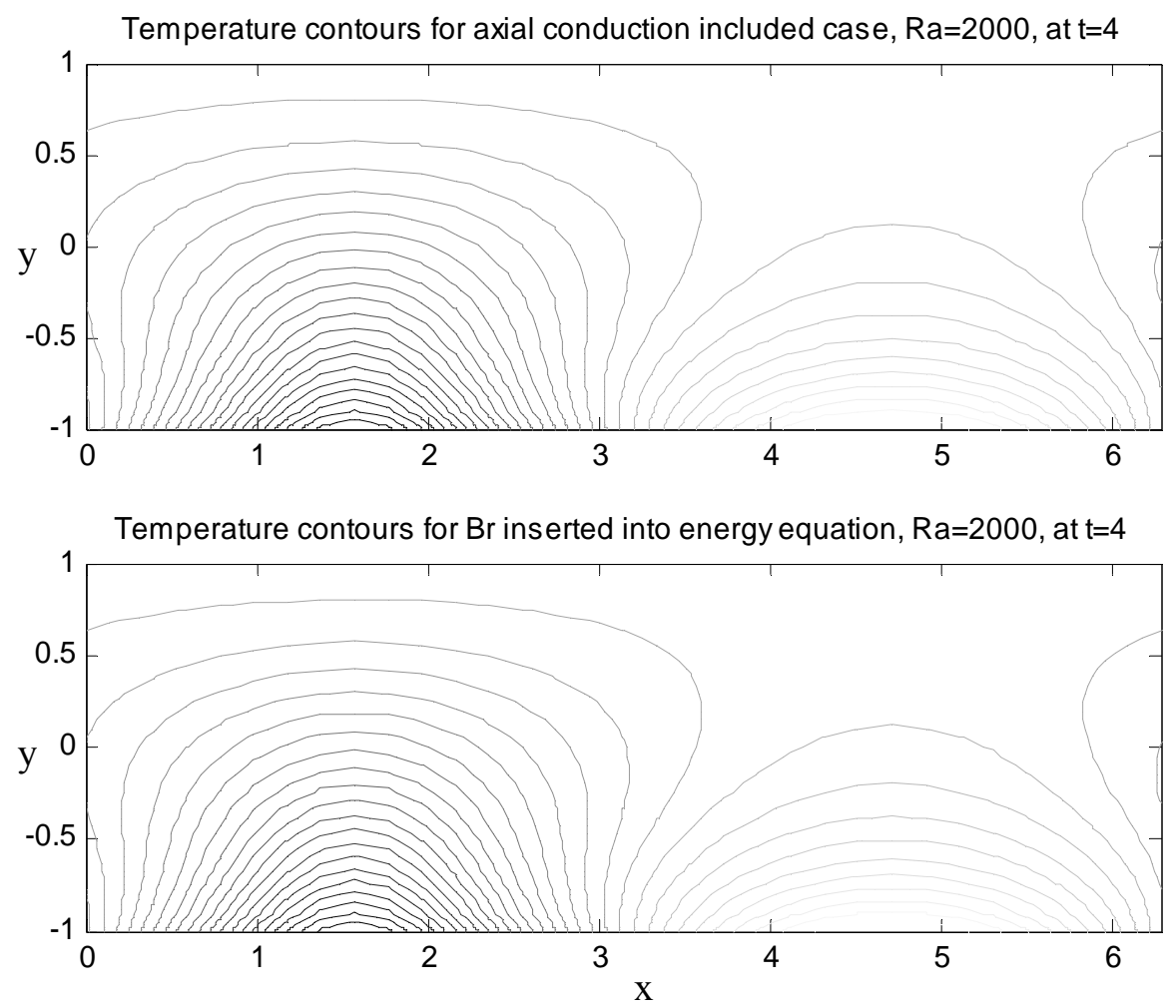

Figure 5.4. Temperature contours for axial cond. included and $\mathrm{Br}$ cases

\subsubsection{Effect of Ra on Natural Convection in Microchannels}

Due to the restrictive nature of the microchannels, two specific Rayleigh numbers are selected for the natural convection, 25.219 and 2000. The reason to select both of these cases is mentioned above. Therefore, after observing for both $\mathrm{Ra}$ numbers the formation of Rayleigh-Benard cells, a comparison should be made between these two specific numbers for the same test case, which in this case $\mathrm{Br}$ inclusion into the energy equation. Figure 5.5 shows the difference between these results. The results indicate that the difference between $\mathrm{Ra}=25.219$ and 2000 is that the cells have a tendency to consolidate, which can be observed from the figure. In Figure 5.5, for $\mathrm{Ra}=25.219$ shows a regular pattern with separate cells. 
However, for $\mathrm{Ra}=2000$ shows the indication of unification for seen from the two cells formed at the entrance side of the channel.
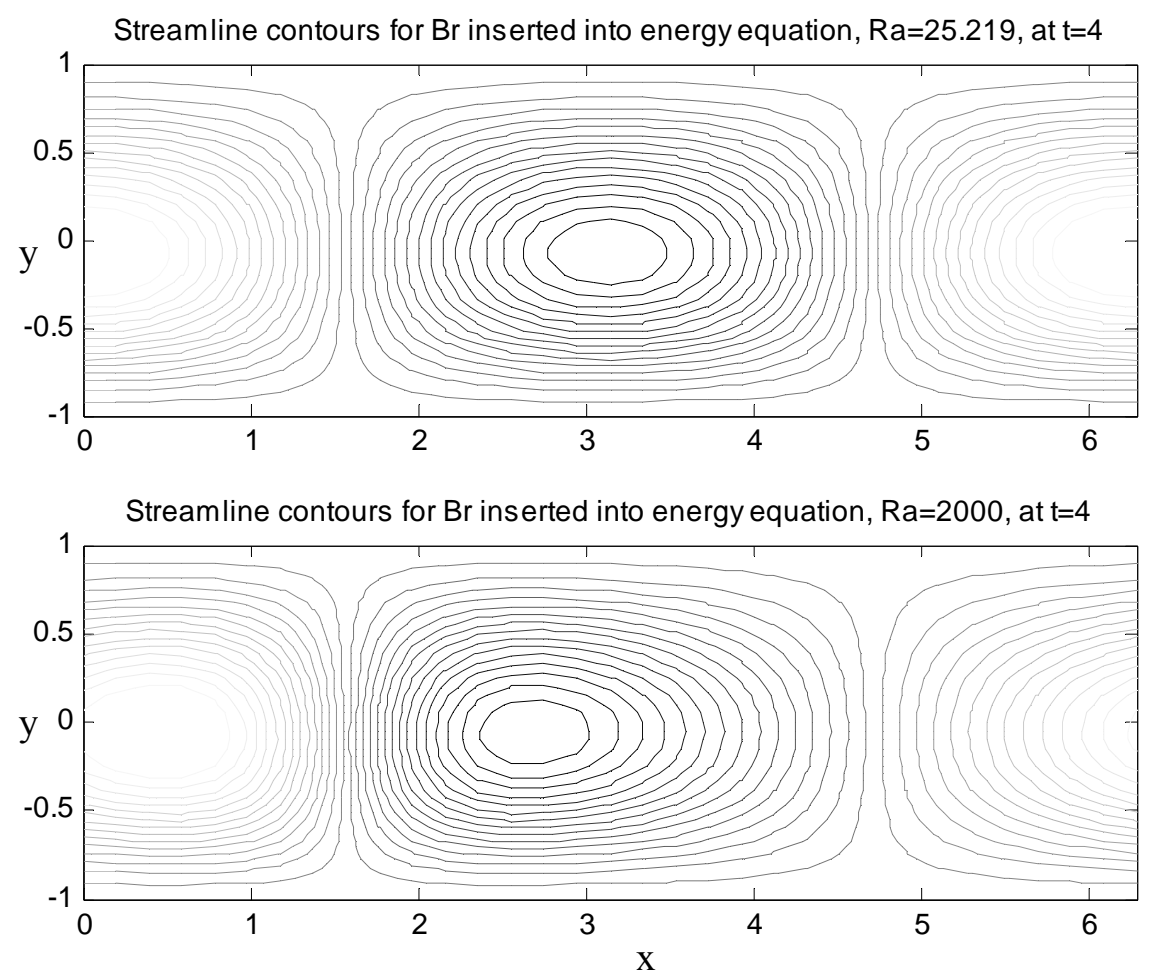

Figure 5.5. Streamline contours for $\mathrm{Ra}=25.219$ and $\mathrm{Ra}=2000$

\subsection{Results and Discussion for 3D}

When the channel is heated from the bottom uniformly 3D structures are expected to form. The motivation for the $3 \mathrm{D}$ work is arising basically from the widely accepted literature for conventional channels. We tried to see if the physics of the Rayleigh-Benard cells for 2D and 3D are the same for micro cases as well. 
Actually, the observed roll pattern is just one of the possibilities. Among the cells observed experimentally under varying conditions, the following two types of cells are mostly found:

Two-dimensional rolls: A particularly simple pattern occurs when all the quantities depend on only one of the horizontal direction, say $\mathrm{x}$. In this case, the cells are infinitely elongated and it is more appropriate to call them rolls, shown in Figure 5.6 a.

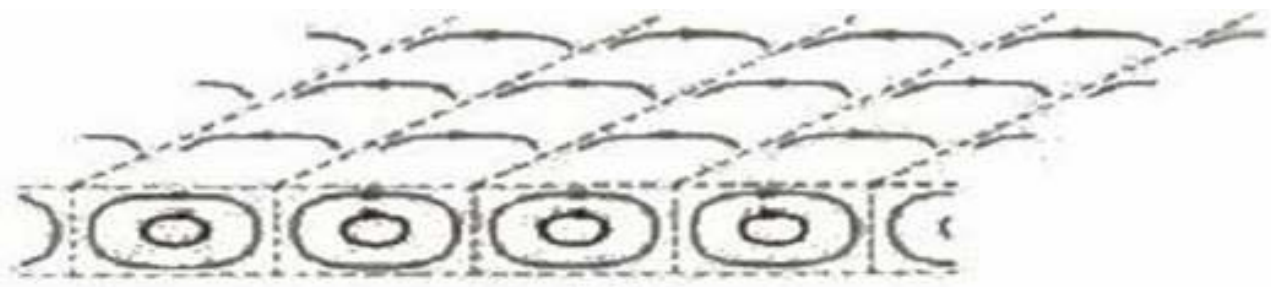

(a)

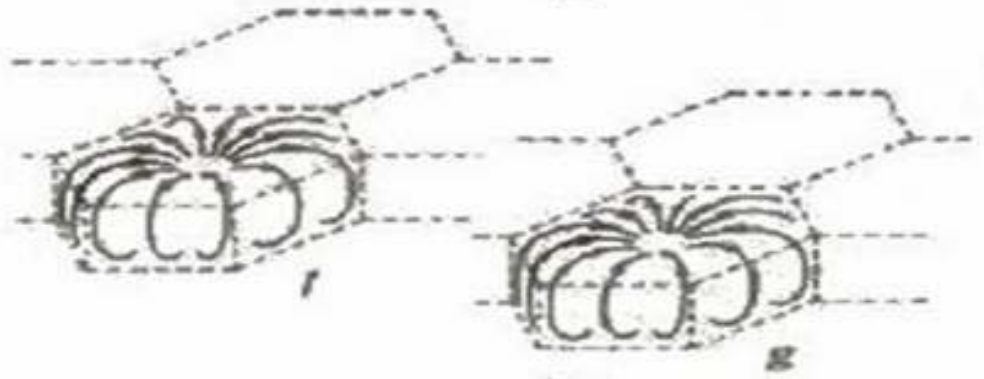

(b)

Figure 5.6. Schematic diagram of convection cells a)Two dimensional rolls b) Hexagonal $l$ - and $g$-cells

Hexagonal cells: This system is a superposition of three roll sets with wave vectors having the same modulus $\mathrm{k}$. A hexagonal cell is called an $l$ - or a $g$-cell depending on the sign of the velocity (i.e., on whether the fluid ascends or descends in the central part of the cell). g-cells are usually observed in the gases and l-cells are seen often in the liquid, shown in Figure $5.6 \mathrm{~b}$. 


\subsubsection{Effect of $\mathrm{Br}$, $\mathrm{Ra}$ and axial conduction for natural convection}

For $2 \mathrm{D}$ cases inclusion of $\mathrm{Br}$ into energy equation does not affect the flow for natural convection. We intended to be sure about the physical system because of the 3D nature of the Rayleigh-Benard cells mentioned above. Consequently, three cases are compared with each other for the same Ra. And also the effect of different Rayleigh numbers for the same test case is investigated.
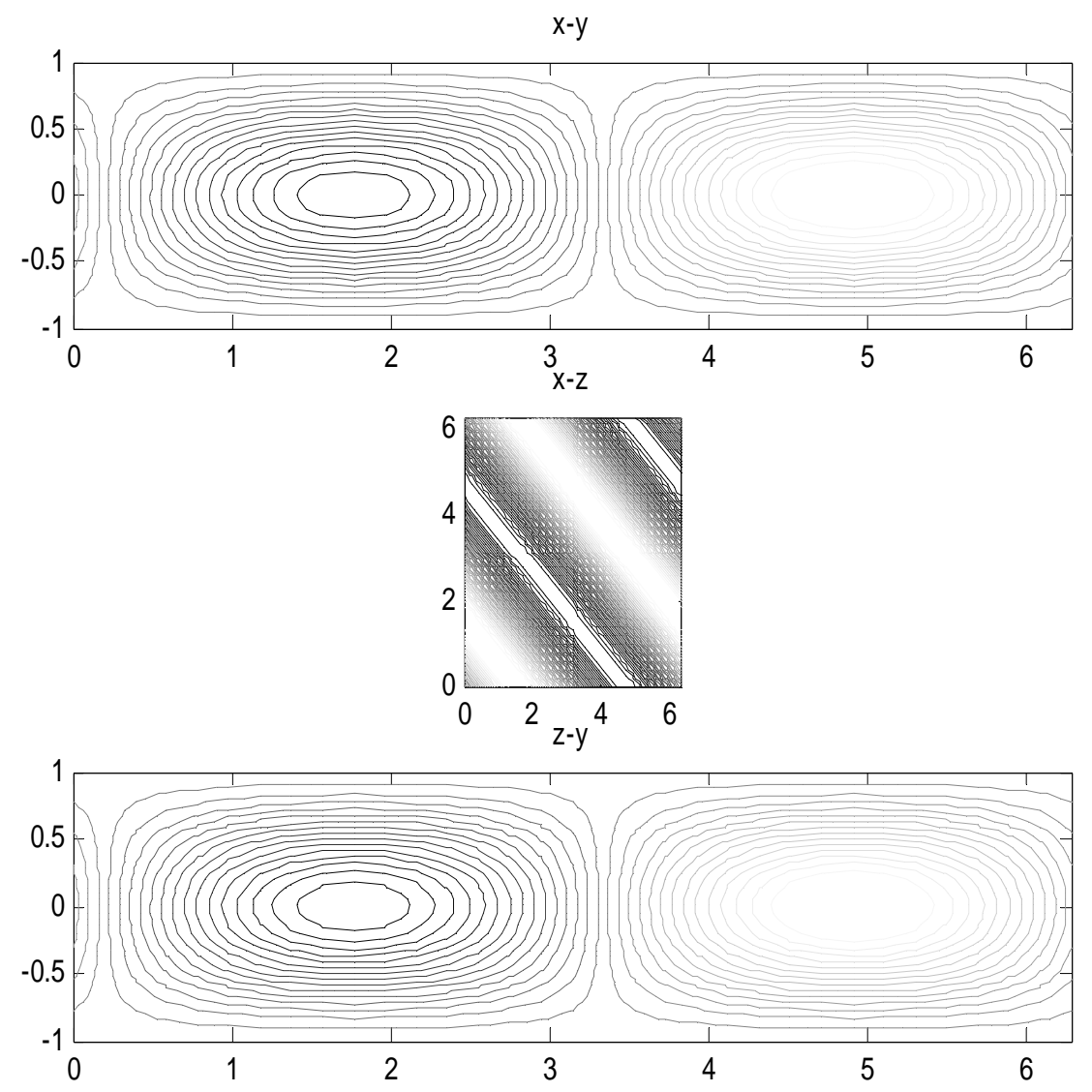

Figure 5.7. 3D Streamline contours for axial conduction included case

For comparison of the three cases, we compare axial conduction and $\mathrm{Br}$ inserted into energy equation case and saw that there are no significant differences 
between these two important cases for natural convection in microchannels for 3D uniformly heated channel. Figures 5.7 and 5.8 show this similarity of these two cases by the help of streamline contour plots. Note that the cells formed are hexagonal in nature.
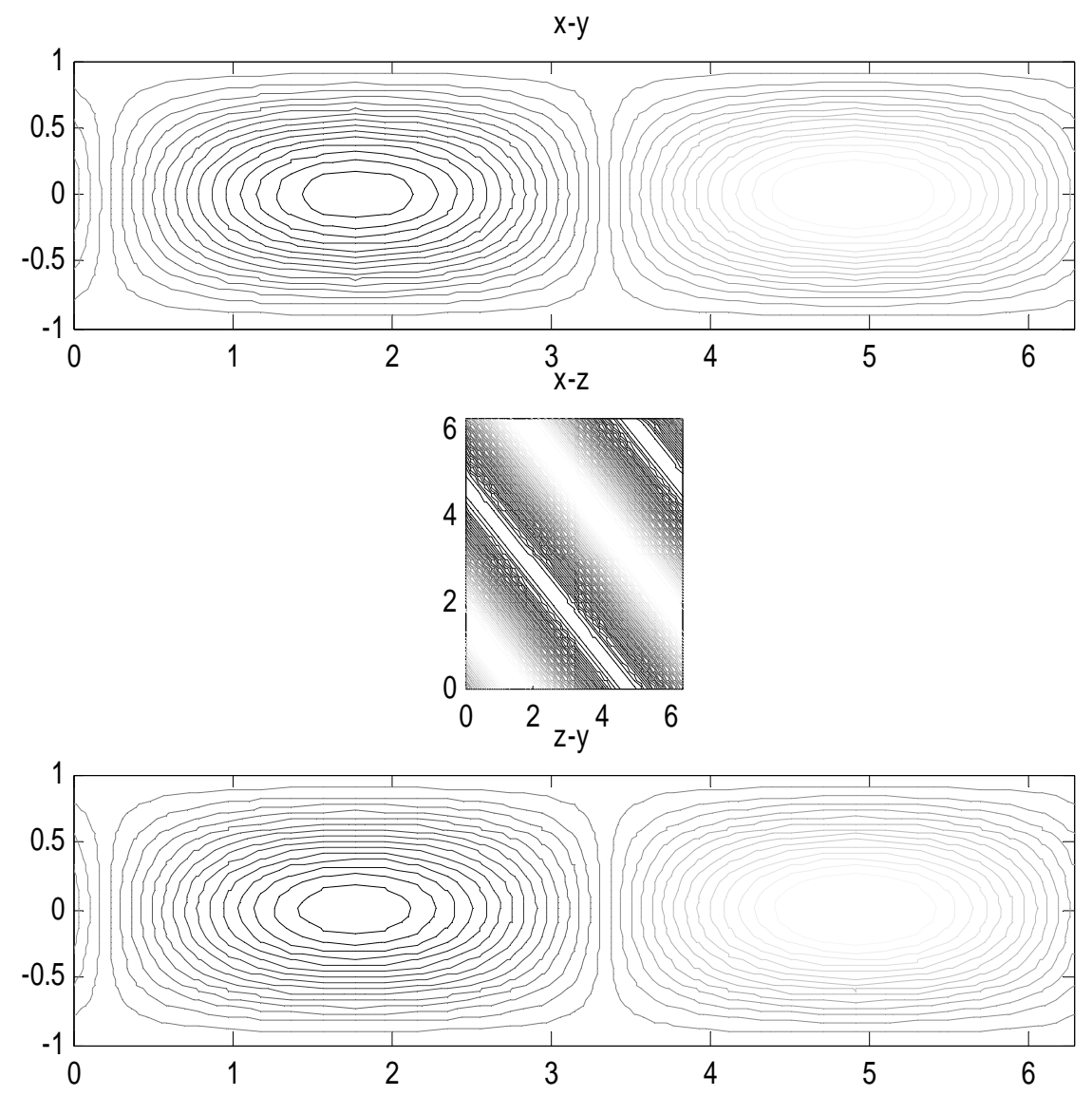

Figure 5.8. 3D Streamline contours for Br included in energy eqn. case

However, when it comes to no-axial conduction case, very interesting but obscure behavior of the fluid is observed. The most important conclusion drawn from the numerical results is the $2 \mathrm{D}$ behavior of the cells on the contrary to the case for the 
energy equation with axial conduction term. We see only two dimensional rolls in Figure 5.9 like the one seen in Figure 5.6 a. The number of the cells also alters from two to four.
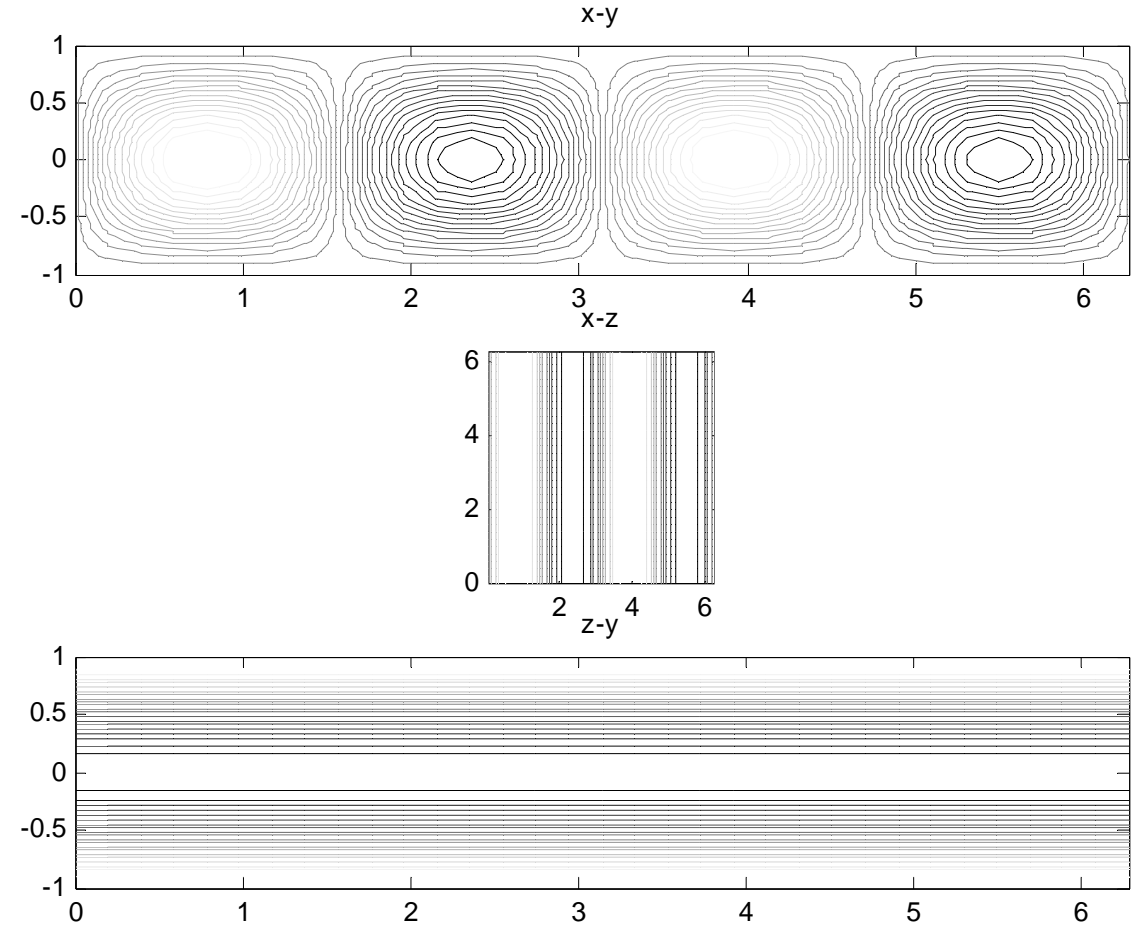

Figure 5.9. 3D Streamline contours for no-axial conduction case

Another observation can be visualized by drawing the plots of non-dimensional temperature results and slicing the plot for the fluid layers to investigate the diffusion of heat into the fluid, Figure 5.10. Figures 5.11 and 5.12 shows also this phenomenon by first slicing it first at $y=-1$ then at $y=-0.25$.

The slices show the 3D nature of the problem. We see the effect of axial conduction from the Figures 5.11 and 5.12. The 3D nature of the cells is seen in Figure 5.10 for other two cases. 


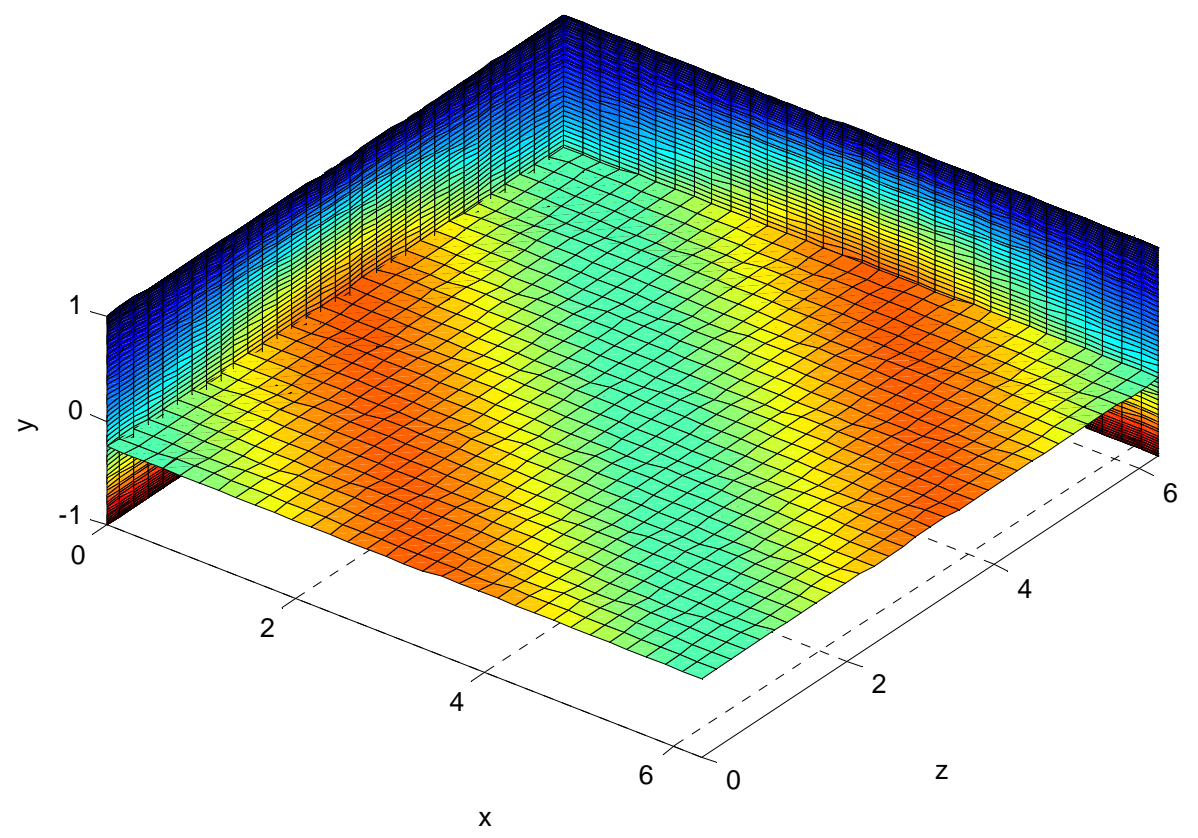

Figure 5.10. Dimensionless temperature sliced at $y=-0.25$ for axial conduction included case 


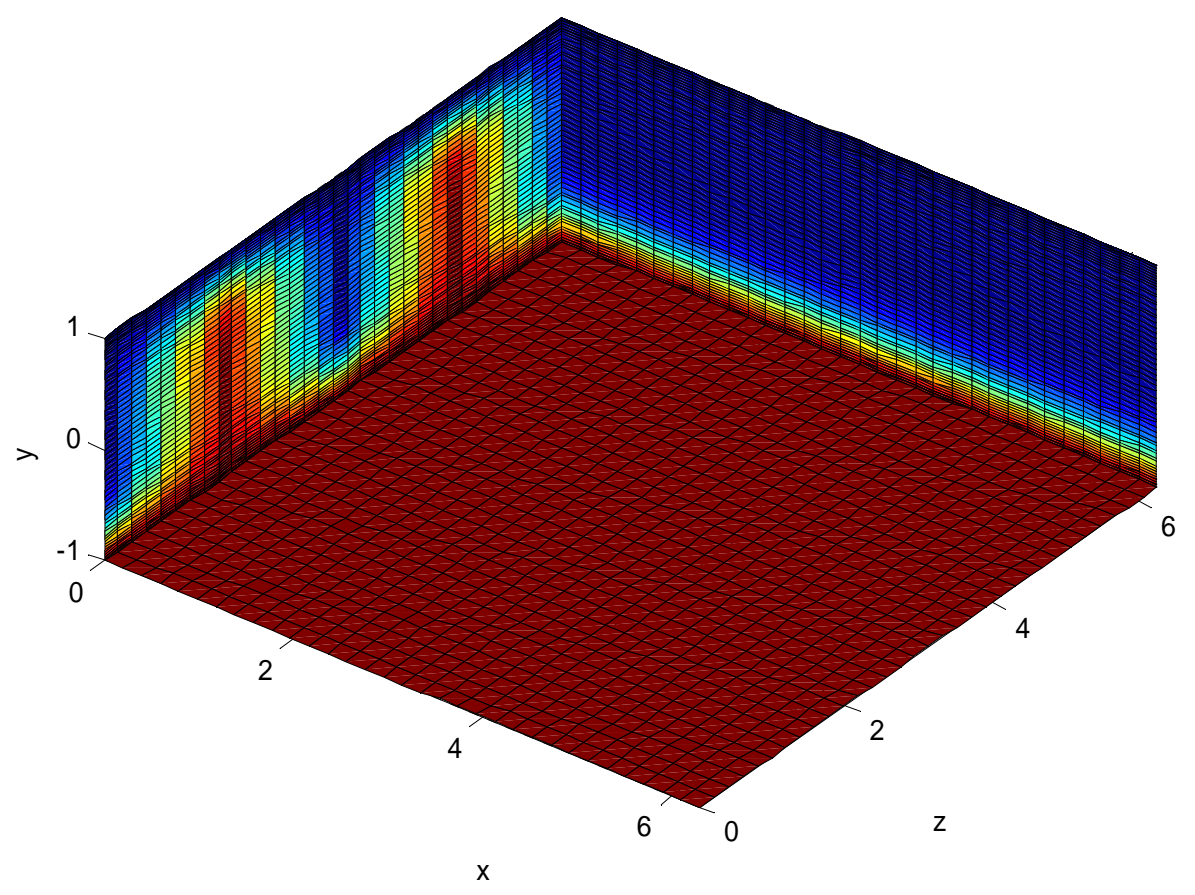

Figure 5.11. Dimensionless temperature sliced at $y=-1$ for no-axial conduction included case 


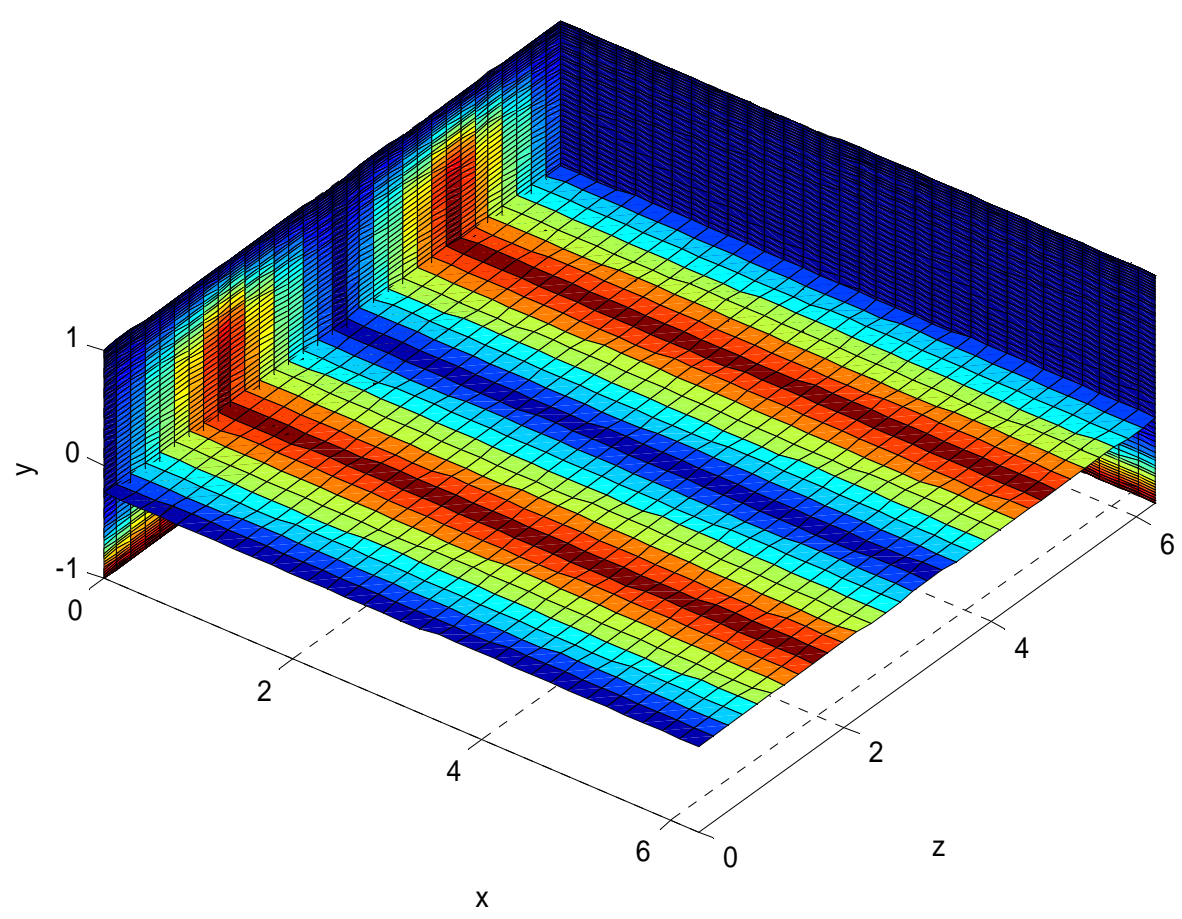

Figure 5.12. Dimensionless temperature sliced at $y=-0.25$ for no-axial conduction included case 


\section{CHAPTER 6}

\section{CONCLUSION}

The purpose of this study was to simulate laminar liquid flow in a microchannel that is heated from below, using DNS and to observe different characteristics of the flow compared to the macrochannel case. There is no similar work for liquids through parallel plate microchannels in the literature, most probably due to the difficulties associated with introduction of $\mathrm{Br}$ in Navier-Stokes equations. Using its definition, Eqn. 2.1, Brinkman number is introduced into the energy equation. The spectral methods based Navier-Stokes solver is modified to include this modification. Due to the nature of Spectral Methods solutions, the solver can only be used for the aforementioned simple geometry. The geometry represents a fully developed middle section of an infinitely long and infinitely wide channel between two parallel horizontal plates. The formulation allows only prescribed constant properties as well as a prescribed Reynolds number. Therefore, the computer code is not suitable for including property variations that were observed in some of the experimental studies. The same limitation is also present for the numerical studies in the literature that are for gas flow through microchannels. However, as it is stated in [10], for the experimentation with constant wall temperature boundary condition, choosing a constant $\mathrm{Br}$ along the flow could be a reasonable approximation. Therefore, by changing $\mathrm{Br}$ from one run to the other, its effects can be observed.

The selection of very low Brinkman numbers in the present study reduces the importance of viscous dissipation, thus temperature variations become small. The results obtained in the selected range qualitatively matches with the experimental results in the literature. There are very limited data in the literature to compare the results of this study. The present work is for parallel plate microchannel and 
includes natural convection for a completely fully developed flow. Many of the experimental results contain entrance effects which may easily be the cause of the deviation of temperature field from the pure numerical results when entrance and fully developed regions are considered together. At this point, the present study tries to show the pure effect of the smaller Br number on the microchannel flow numerically. Considering this aim, it is seen at the end of the results that secondary effect of the $\mathrm{Br}$ is observed for the single-phase convective heat transfer at the temperature gradient level. It is definitely not the case for the primary effect of $\mathrm{Br}$ which directly affects the temperature field with its relatively higher values in the energy equation. This may easily be seen for gaseous flow for constant heat flux case [20]. Another reason for the differences between experimental and numerical values can be the fact that it is impossible to introduce surface roughness to our simulated geometry that seems to be the main obstacle in front of having quantitative comparisons with the experimental results. Some authors have suggested that the deviations from macroscale theory that have been observed may be due to the large relative roughness of the microchannel walls. In microchannels, relative roughness becomes very high due to the small hydraulic diameter. Moreover, during the micromachining process, the channels become hybrid, with two distinct materials and roughness values, making direct comparison of the microchannel data with traditional theory and numerical work problematic as it is stated in [47].

Regardless of lack of enough experimental data for comparing with the numerical results correctly, our aim is to simulate the unusual behavior of decreasing of $\mathrm{Nu}$ with increasing $\mathrm{Re}$ in the laminar regime of microchannels and to show that $\mathrm{Br}$ can be introduced to explain this strange behavior numerically. We know that since $\mathrm{Br}$ shows the relative importance of viscous dissipation, it plays different roles for the cases of fluid heated and cooled. For the fluid being heated, the exponent $d$ of $B r$ in the Eqn. 4.11 should be positive, because the $\mu V^{2}$ term also tends to increase the coolant temperature and so the Nusselt number. At a constant velocity, Re increases due to reduction in the coolant viscosity because 
of the temperature rise of the fluid simulated by taking different $\mathrm{Re}$ into consideration for the same case with constant mean velocity. Therefore, the $\mu V^{2}$ term reduces and $\left(\mathrm{Re}^{0.62} \mathrm{Br}^{d}\right)$ decreases. This is the explanation of the decrease of $\mathrm{Nu}[2,3]$ in spite of the increase in values of Re, which is just the opposite of the behavior seen in conventional channels when entrance effects are taken into consideration.

According to the results observed from the case " $\mathrm{Br}$ inserted into the energy equation", the following conclusions are drawn:

- For the case of fluid heated, the exponent of $\mathrm{Br}$ in Eqn. 4.11 is positive and the results are in agreement with the experimental results [10],

- Analysis with the inputs from the literature shows that besides Re, Pr and geometrical parameters, $\mathrm{Nu}$ correlates with $\mathrm{Br}$ in the form of Eqn. 4.11. This correlation is investigated in this numerical work and we observed that when simulating the flow through microchannels numerically, importance should be given to the effect of viscous dissipation, therefore $\mathrm{Br}$.

- The unusual behavior of $\mathrm{Nu}$ decreasing with Re increasing in the laminar regime, especially less than $\operatorname{Re}<700$, can be explained by Br numerically.

- The Brinkman number correlates the convection in spite of its relatively low values. Therefore, the same observation with [32] is drawn: Even a small amount of viscous dissipation causes an increase in the values of $\mathrm{Nu}$ for the numerical set-up with the walls maintained at constant wall temperature. However, when the values of $\mathrm{Br}$ get much smaller, the correlation between $\mathrm{Nu}$ and $\mathrm{Br}$ cannot be observed anymore.

- When $\mathrm{Br}$ is large, it affects $\mathrm{T}_{\mathrm{f}}$ directly due to the effect of viscous dissipation, even for a constant value of $\mathrm{Br}$ along the flow.

- More data are required to obtain a universal correlation even though comparison with limited data in the literature supports the inclusion of $\mathrm{Br}$. 
For "no-axial conduction" runs, in $\mathrm{Re}=80,87$ and 97, partially formed convection cells are observed. These structures are not present in the runs with axial conduction and in the runs with $\mathrm{Br}$ for those Reynolds numbers. That unusual behavior shows that axial conduction plays an important role in low Re liquid flows and it should be included in formulations for low Re microchannel simulations. This observation perfectly matches with the other similar conclusions in the literature [32]. The axial conduction term becomes negligible at higher values of Re (higher Pe).

Considering natural convection in microchannels; this work is the very first work in the literature. Therefore, the critical numbers are chosen generally according to conventional channels. However, because of the strong effect of size of microchannels on $\mathrm{Ra}$ for the flow through microchannels, we cannot run the computer code for higher magnitudes.

The conclusions drawn for natural convection in microchannels can be expressed as follows:

- There is not a significant difference between the "axial conduction inclusion" and "Brinkman number inserted into the energy equation" cases for periodically heated lower wall configuration. The results from the temperature contours also support the conclusion.

- With the help of artificial temperature field disturbance created by the program we were able to simulate cell formations even for low Ra values. We concluded that in nature since these disturbances occur naturally, we can say that formation of cells is also likely to occur in microchannels with the help of these alterations.

- When the lower wall is heated periodically, we observe regular patterns, two dimensional rolls. However, for the uniformly heated lower wall boundary condition, hexagonal patterns occur. 
- The effect of axial conduction is seen from the results of "no-axial conduction included" case for uniformly heated lower wall configuration. An important conclusion drawn from the numerical results is the $2 \mathrm{D}$ behavior of the cells in contrast to the case for the energy equation with axial conduction term. 


\section{REFERENCES}

[1] Peng, X. F. and Peterson, G. P., 1996, Convective heat transfer and flow friction for water flow in microchannel structures, Int. J. Heat Mass Transfer, 39, pp. 2599-2608.

[2] Peng, X. F. and Peterson, G. P., 1995, The effect of thermofluid and geometrical parameters on convection of liquids through rectangular microchannels, Int. J. Heat Mass Transfer, 38, pp. 755-758.

[3] Wang B. X. and Peng X. F., 1994, Experimental investigation on liquid forced-convection heat transfer through microchannels, Int. J. Heat Mass Transfer, 37, Suppl. 1, pp. 73-82.

[4] Tuckermann D. B. and Pease R. F., 1991, High Performance heat sinking for VLSI, IEEE Electronic Device Lett., 2, pp. 126-129.

[5] Tuckermann D. B. and Pease R. F., 1982, Optimized convective cooling using micromachined structure, J. Electrochem. Soc., 129, pp. C98.

[6] Wu P. and Little W. A., 1983, Measurement of friction factor for the flow of gases in very fine channels used for microminiature Joule-Thomson refrigerators, Cyrogenics, 24, pp. 273-277.

[7] Pfahler J., Harley J., Bau H. H. and Zemel J., 1990, Liquid transport in micron and submicron channels, J. Sensors Actors, A21-23, pp. 431-434. 
[8] Choi B., Barron R. F. and Warrington R. O., 1991, Liquid flow and heat transfer in microtubes, Micromechanical Sensors, Actuators and Systems (Edited by D. Cho. et al.), ASME DSC 32, pp. 123-134.

[9] Peng X. F. and Wang B. X., 1995, Experimental investigation of heat transfer in flat plates with rectangular microchannels, Int. J. Heat Mass Transfer, 38, pp. 127-137.

[10] Tso C. P. and Mahulikar S. P., 1998, The use of the Brinkman number for single phase forced convective heat transfer in microchannels, Int. J. Heat Mass Transfer, 41, pp. 1759-1769.

[11] Tso C. P. and Mahulikar S. P., 2000, Experimental verification of the role of Brinkman number in microchannels using the local parameters, Int. J. Heat Mass Transfer, 43, pp. 1837-1849.

[12] Peng X. F. and B.X. Wang B. X., 1993, Forced convection and flow boiling heat transfer for liquid flowing through microchannels, Int. J. Heat Mass Transfer, 36, pp. 3421-3427.

[13] Peng X. and Peterson G., 1996, Forced convection heat transfer of singlephase binary mixtures through microchannels, Experimental Thermal and Fluid Science, 12, pp. 99-104.

[14] Yang C. and Webb R., 1996, Condensation of R12 in small hydraulic diameter extruded aluminium tubes, Int. J. Heat Mass Transfer, 39, pp. 791-800.

[15] Incropera F. and DeWitt D., 1994, Fundamentals of Heat and Mass Transfer, fourth ed., John Wiley and Sons, New York. 
[16] Garimella S., Dowling W., Veen M.V.D. and Killion J., 2001, The efect of simultaneously developing flow on heat transfer in rectangular tubes, Heat Transfer Engineering, 22, pp. 12-25.

[17] Gnielinski V., 1976, New equations for heat and mass transfer in turbulent pipe and channel flow, International Chemical Engineering, 16, pp. 359-368.

[18] Shah R. K. and London A. L., 1978, Laminar Flow Forced Convection in Ducts, Academic Press, New York.

[19] Tunç, G. and Bayazitoğlu, Y., 2002, Convection at the entrance of micropipes with sudden wall temperature change, Proceedings of IMECE 2002, November 17-22, New Orleans, Lousiana.

[20] Tunç, G. and Bayazıtoğlu, Y., 2001a, Heat transfer in microtubes with viscous dissipation, Int. J. Heat Mass Transfer, 44 (13), pp. 2395-2403

[21] Xu B., Ooi K. T., Mavriplis C. and Zaghloul M. E., 2003, Evaluation of viscous dissipation in liquid flow in microchannels, J. Micromech. Microeng. ,13, pp. 13-57.

[22] Guo Z. Y. and Li Z. X., 2003, Size effect of microscale single phase flow and heat transfer, Int. J. of Heat and Mass Transfer, 46, pp. 149-159.

[23] Mala G. M. and Li D., 1999, Flow characteristic of water in microtubes, Int. J. of Heat and Fluid Flow, 20, pp. 142-148

[24] Mala G. M., Li D. and Qu W., 2000, Heat transfer for water flow in trapezoidal silicon microchannels, Int. J. of Heat and Mass Transfer, 43, pp. 3925-3936. 
[25] Sobhan C. B. and Garimella S. V., 2001, A comparative analysis of studies on heat transfer and fluid flow in microchannels, Microscale Thermophysical Engineering, 5, pp. 293-311.

[26] Palm B., Heat transfer in microchannels, 2001, Microscale Thermophysical Engineering, 5, pp. 155-175.

[27] Koo J. and Kleinstreuer C., 2004, Viscouss dissipation effects in microtubes and microchannels, Int. J. of Heat and Mass Transfer, 47, pp. 3159-3169.

[28] Morini G. L. , Spiga M. and Tartarini P., 1998, Laminar viscous dissipation in rectangular ducts, Int. Comm. Heat Mass Transfer, Vol., 25, No. 4, pp. 551560.

[29] Larrode F. E., Housiadas C. and Drossinos Y., 2000, Slip-flow heat transfer in circular tubes, Int. J. of Heat and Mass Transfer, 43, pp. 2669-2680.

[30] Tiselj I., Hetsroni G., Mavko B., Mosyak A., Pogrebnyak E.and Segal Z., 2004, Effect of axial conduction on heat transfer in micro-channels, Int. J. of Heat and Mass Transfer, 47, pp. 2551-2565.

[31] Koo J. and Kleinstreuer C., 2003, Liquid flow in microchannels: experimental observations and computational analysis of microfluidics effects, $J$. Micromech. Microeng., 13, pp. 568-579.

[32] Kuznetsov A. V., Xiong M. and Nield D. A., 2003, Thermally developing forced convection in a porous medium: Circular duct with walls at constant temperature with longitudinal conduction and viscous dissipation effects, Transport in Porous Media, 53, pp. 331-345. 
[33] Kuznetsov A. V., Xiong M. and Nield D. A., 2004, Effect of viscous dissipation and flow work on forced convection in a channel filled by a saturated porous medium, Transport in Porous Media, 56, pp. 351-367.

[34] Morini G. L., 2000, Analytical determination of the temparature distribution and Nusselt numbers in rectengular ducts with constant axial heat flux, Int. J. of Heat and Mass Transfer, 43, pp. 741-755.

[35] Warrier G. R., Dhir V. K. and Momoda L. A., 2002, Heat transfer and pressure drop in narrow rectangular channels, Experimental Thermal and Fluid Science, 26, pp. 53-64.

[36] Paputsky I. , Ameel T. and Frazier A. B., 2001, A review of laminar single phase flow in microchannels, Proceedings of 2001 ASME IMECE November 11$16, \mathrm{NY}$

[37] Weisberg A., Bau H. H. and Zemel J. N., 1992, Analysis of microchannels for integrated cooling, Int. J. Heat Mass Transfer, 35, pp. 2465-2474.

[38] Dutta P., Beşkök A. and Warburton T. C., 2002, Numerical Simulation of Mixed Electroosmotic/Pressure Driven Microflows, Numerical Heat Transfer, Part A, 41, pp. 131-148.

[39] Kakaç S. and Yener Y., 1995, Convective Heat Transfer, CRC Press, Florida.

[40] Kuznetsov A. V., Xiong M. and Nield D. A., 2003, Thermally Developing Forced Convection in a Porous Medium: Circular Duct with Walls at Constant Temperature, with Longitudinal Conduction and Viscous Dissipation Effects, Transport in Porous Media, 53, pp. 331-345 
[41] Kim J., Moin P., Moser R., 1987, Turbulence statistics in fully developed channel at low Reynolds number, J. Fluid Mechanics, Vol. 17, pp.133.

[42] Zhang S.Q., 1991, Numerical simulations of mixed convection in a spatially periodically heated channel, M.Sc. thesis, Northeastern University, Boston.

[43] Tar1 I., 1998, Spherical particle motion simulation by spectral methods, Ph.D thesis, Northeastern University, Boston.

[44] Greengard L., 1988, Spectral integration and two-point boundary value problems, Reserch report at Yale University

[45] Guo Z.Y. and Li Z.X., 2003, Size effect on single phase channel flow and heat transfer at microscale., Int. J. Of Heat and Fluid Flow, 24, pp. 284-298.

[46] Peng X. F. , Peterson G. P. and Wang B. X., 1994, Frictional flow characteristic of water flowing through microchannels, Experimental Heat Transfer, 7, pp. 240-264.

[47] Paputsky I., Ameel T. and Frazier B. A., 2001, A review of laminar singlephase flow in microchannels, 2001 ASME IMECE. 